\title{
A SYSTEM FOR MEASURING THE LIFT AND DRAG FORCES OF A SPINNING GOLF BALL HELD FIXED WITHIN A WIND TUNNEL
}

\author{
A Thesis \\ Presented to \\ the Faculty of California Polytechnic State University \\ San Luis Obispo
}

\author{
In Partial Fulfillment \\ of the Requirements for the Degree \\ Master of Science in Mechanical Engineering
}

by

Ryan Miller

February 2009 
(c) 2009

Ryan Miller

ALL RIGHTS RESERVED 


\section{COMMITTEE MEMBERSHIP}

TITLE:

AUTHOR:

DATE SUBMITTED:

COMMITTE CHAIR: Tom Mase

COMMITTEE MEMBER: Kim Shollenberger

COMMITTEE MEMBER: John Fabijanic
A SYSTEM FOR MEASURING THE LIFT AND DRAG FORCES OF A SPINNING GOLF BALL HELD FIXED WITHIN A WIND TUNNEL

Ryan Miller

February 2009 


\begin{abstract}
A SYSTEM FOR MEASURING THE LIFT AND DRAG FORCES OF A SPINNING GOLF BALL HELD FIXED WITHIN A WIND TUNNEL

by

Ryan Miller
\end{abstract}

A system was designed, built and tested in order to test the aerodynamic properties of a standard golf ball in a wind tunnel manufactured by ELD, Inc. model 406(B). The system consists of a rotating shaft, on which the golf ball is attached, connected to a two-axis force transducer. Additionally, an automated data acquisition system was built for enhanced precision of measurements. Data for wind speeds up to $160 \mathrm{ft} / \mathrm{s}$ and rotational speeds up to 8, $600 \mathrm{rpm}$ were obtained and analyzed. The purpose of the designed apparatus was to allow for studies to better understand the lift and drag coefficients of golf balls during their flight. Subsequent to testing, it was found that the force transducer was not adequate to measure the lift and drag coefficients with sufficient accuracy. Several suggestions have been made on how to improve the wind tunnel so that better results might be obtained in the future. 


\section{Acknowledgements}

The author wishes to thank Dr. Tom Mase for all the support and guidance

through the entire research process. Also the author would like to extend special thanks to Dr. Kim Shollenberger and Dr. John Fabijanic for the priceless advice and constructive criticism that really pushed the project forward.

The author would additionally like to thank Chuck Keezer for helping with equipment, Hans Mayer for his many suggestions, and Dr. Glen Thorncroft for advice on building a data acquisition system. 


\section{Contents}

List of Tables viii

List of Figures $\quad$ ix

1 Introduction 1

1.1 Motivation . . . . . . . . . . . . . . . . 3

1.2 Background ...................... . . . 9

2 Experimental Apparatus $\quad 14$

2.1 Wind Tunnel . . . . . . . . . . . . . . . . . . . . . . 14

2.2 Sting . . . . . . . . . . . . . . . . . 18

2.3 Fixture . . . . . . . . . . . . . . . . . 19

2.4 Data Acquisition System . . . . . . . . . . . . . . . . 24

3 Experimental Procedure $\quad 28$

4 Results $\quad 33$

4.1 Lift and Drag Results . . . . . . . . . . . . . . . . . . . . . . 33

4.2 Vortex Shedding . . . . . . . . . . . . . . . 37

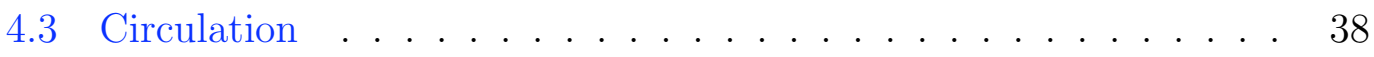

4.4 Areas For Improvement . . . . . . . . . . . . . . . . . . . . . . . . 39

4.4.1 Fairing Gives False Readings . . . . . . . . . . . . . . . . . 40

4.4 .2 Noise From the Fan Motor . . . . . . . . . . . . . . . . . . 42

4.4 .3 Solutions..................... 43

5 Suggestions $\quad 45$

5.1 Pitot Tube Mount . . . . . . . . . . . . . . . . . 45 
5.2 Pitot Tube Slit . . . . . . . . . . . . . . . . . . . . 46

5.3 Bernoulli Effect . . . . . . . . . . . . . . . . 47

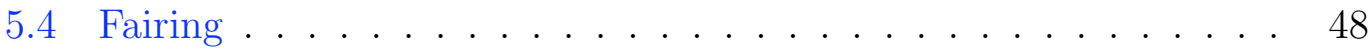

5.5 Mechanical Interference . . . . . . . . . . . . . . . . . . . 49

5.6 Suction Through Fairing . . . . . . . . . . . . . . . 50

5.7 Force Transducer Isolation . . . . . . . . . . . . . . . . . . . 53

5.8 Hot Wire Anemometer . . . . . . . . . . . . . . . . . . . 54

6 Conclusion $\quad 56$

$\begin{array}{ll}\text { References } & 59\end{array}$

$\begin{array}{ll}\text { A Drawings } & 65\end{array}$

A.1 Final Concept . . . . . . . . . . . . . . . . . . 65

A.2 Initial Concept . . . . . . . . . . . . . . 70

$\begin{array}{ll}\text { B Mathematics } & 80\end{array}$

B.1 Bernoulli Equation . . . . . . . . . . . . . . 80

B.2 Uncertainty Analysis . . . . . . . . . . . . . . . . . 81

$\begin{array}{ll}\text { C Software Tools } & 85\end{array}$

$\begin{array}{ll}\text { D Calibration } & 90\end{array}$ 


\section{List of Tables}

1.1 Independent functions suggested for curve fitting of golf balls' tra-

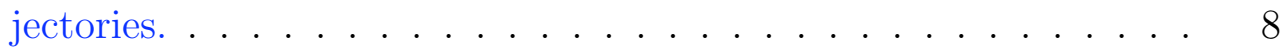

2.1 Bill of materials for the final design of the test apparatus. . . . . 23

2.2 Comparison of standard deviations for some typical conditions. . . 26

3.1 Input Channels. . . . . . . . . . . . . . . . . . . . 29 


\section{List of Figures}

1.1 Device Model . . . . . . . . . . . . . . . . . . . . . . . . . . . 2

1.2 Typical Golf Ball Trajectory . . . . . . . . . . . . . . . . . 5

1.3 Flight Coefficients vs Reynolds Number . . . . . . . . . . . . 6

1.4 Flight Coefficients vs Spin Number . . . . . . . . . . . . . 6

1.5 Historical Lift and Drag Coefficients Results . . . . . . . . . . 9

1.6 Velocity Profile of Perturbed Poiseuille Flow . . . . . . . . . . 13

2.1 The Wind Tunnel . . . . . . . . . . . . . . . . . . . . . . . . . 15

2.2 Free Stream Velocity Profile, Coarse . . . . . . . . . . . . . 16

2.3 Free Stream Velocity Profile, Fine . . . . . . . . . . . . . 16

2.4 Pitot Tube . . . . . . . . . . . . . . . . . . . . . 17

2.5 Linear Variable Differential Transformer (LVDT) Cutaway . . . . 19

2.6 Calibration Dial . . . . . . . . . . . . . . . . . . . . . . . . 20

2.7 Test Fixture for Rotating Golf Ball _ . . . . . . . . . . . . 21

2.8 Nylon Gear . . . . . . . . . . . . . . . . . . . . . . . . . . . 21

2.9 Force Balance . . . . . . . . . . . . . . . . . . . . . . . . . . . . 23

2.10 DAQ Breadboard . . . . . . . . . . . . . . . . 27

2.11 Strobotac . . . . . . . . . . . . . . . . 27

3.1 Calibration Equipment . . . . . . . . . . . . . . . . . . . . . . 29

4.1 Lift Force . . . . . . . . . . . . . . . . . . 35

4.2 Coefficient of Lift . . . . . . . . . . . . . . . . 35

4.3 Drag Force . . . . . . . . . . . . . . . . . . . 36 
4.4 Coefficient of Drag . . . . . . . . . . . . . . . . 37

4.5 Centroid Velocity vs. Tangential Velocity . . . . . . . . . . . . 39

4.6 Fairing Drag Forces . . . . . . . . . . . . . . . . . . . . . . 41

4.7 Fairing Lift Forces . . . . . . . . . . . . . . . . . . 42

4.8 Typical Standard Deviation _ . . . . . . . . . . . . . . 43

5.1 Pitot Tube Mount . . . . . . . . . . . . . . . . . . 46

5.2 Pitot Tube Blockage . . . . . . . . . . . . . . . 47

5.3 Bernoulli Effect on Sting . . . . . . . . . . . . . . . . . . 49

5.4 Cardboard Fairing . . . . . . . . . . . . 50

5.5 Mechanical Interference Comparison _ . . . . . . . . . . 51

5.6 Suction Through Fairing . . . . . . . . . . . . . . . . . . 52

5.7 An Air-tight Fairing . . . . . . . . . . . . . . . . 53

5.8 Standard Deviation Comparison . . . . . . . . . . . . . 54

C.1 LabVIEW program GUI . . . . . . . . . . . . . . 86

C.2 LabVIEW Block Diagram, Recording . . . . . . . . . . . 87

C.3 LabVIEW Block Diagram, Streaming . . . . . . . . . . . . . 88

C.4 LabVIEW Hardware Panel . . . . . . . . . . . . . . . . . . . 89

D.1 Drag Calibration Curve. . . . . . . . . . . . . . . . . . . 91

D.2 Lift Calibration Curve . . . . . . . . . . . . . . . . . . 91

D.3 Pressure Calibration Curve . . . . . . . . . . . . . . . . 92 


\section{Nomenclature}

$\alpha \quad$ angle between the velocity vector and ground

$\beta \quad$ Orr-Sommerfeld constant

U mean free-stream velocity

u velocity vector

$\kappa \quad$ dipole strength

$\mu \quad$ viscosity

$\nu \quad$ kinematic viscosity

$\omega \quad$ rotational speed

$\phi \quad$ stream function

$\rho \quad$ density

$\theta \quad$ angle of launch relative to ground

$\tilde{\mathbf{U}}$ fluctuation of free-stream velocity

$\tilde{\rho} \quad$ fluctuation of density

$\tilde{p} \quad$ fluctuations in pressure 


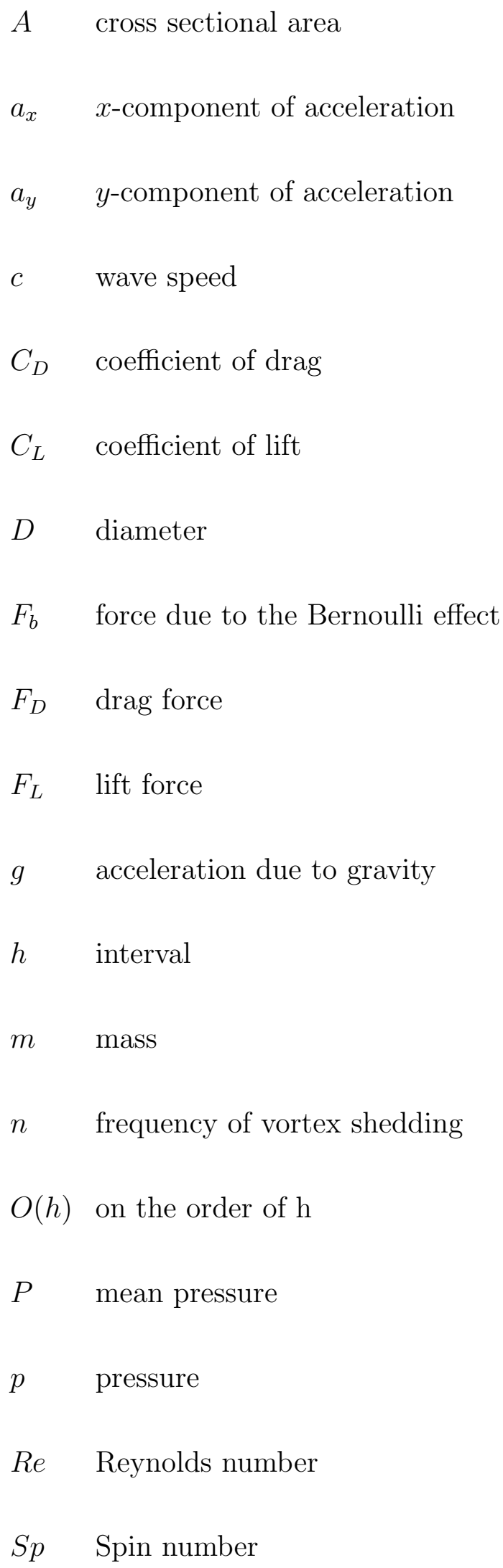




St $\quad$ Strouhal number
$t \quad$ time
$u(x) \quad$ any continuously differentiable function of $\mathrm{x}$
$U(z) \quad$ Poiseuille flow velocity profile
$V \quad$ speed
$W \quad$ weight




\section{Chapter 1}

\section{Introduction}

A golf ball is an interesting aerodynamic object since it may be thought of as a rotating, translating sphere with a complex surface used to induce turbulence in the boundary layer at low Reynolds numbers. The lift and drag coefficients are typically assumed to be functions of Reynolds number and spin number. The spin number $(S p)$ is a non-dimensional coefficient usually taken to be $S p=\omega D / V$ [33], where $\omega$ is the rotational speed, $D$ is the diameter of the golf ball, and $V$ is the velocity of the golf ball. The Reynolds number is defined in the usual way, $R e=\rho V D / \mu$, where $\rho$ is the density of air, and $\mu$ is the viscosity.

Seeing how the flow field around a golf ball is relatively complex, analytical techniques are of limited use. Even the most modern computational fluid dynamics (CFD) methods (as of 2008) are not quite adequate for analysis [17]. The main troubles lie in the scale of the dimples compared to the ball diameter, the geometry of the dimples, the near-critical Reynolds numbers involved and the

fast rotational speed of the ball. A soccer ball has been successfully examined using the standard $k-\varepsilon$ method [3], but so far the only accurate results are for non-spinning, steady-state models. Naturally then, experimentation is one of 
the key methods for understanding the topic of sports balls aerodynamics. The major piece of equipment conventionally used has been a wind tunnel. Equipped with Pitot tubes and force transducers, the wind tunnel provides large amounts of information. It has its limits, however, and these are addressed in later sections (see Ch. 2).

For the remainder of the paper, the following sign convention will be used for our coordinate system. Imagine that you are inside the wind tunnel's test section looking straight into the oncoming free-stream flow. The $x$-axis points directly into the flow; straight ahead. You would be moving in the $y$-axis if you moved side-to-side, and the $z$-axis is the vertical direction.

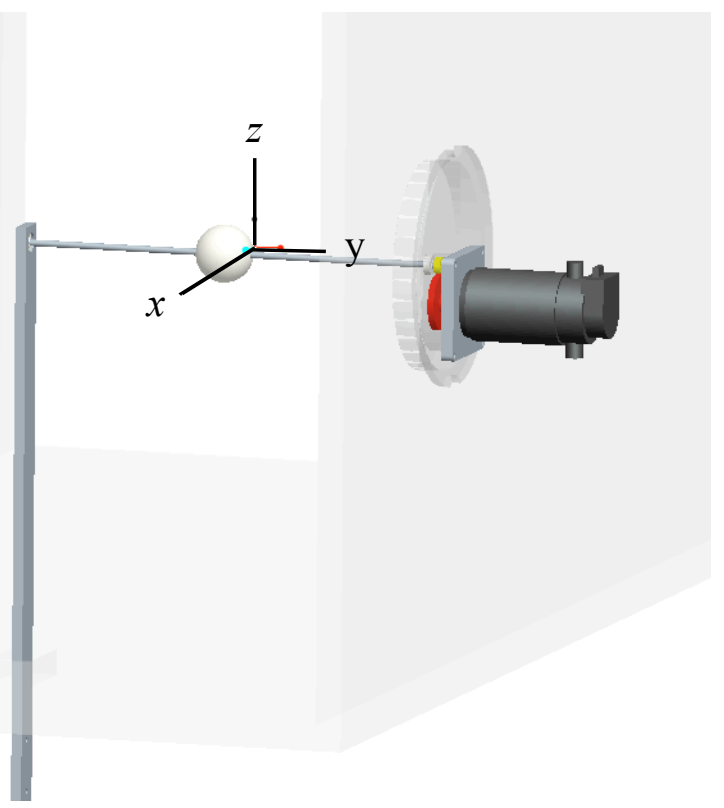

Figure 1.1: This is a 3-D model of the testing system, created using SolidWorks. 


\subsection{Motivation}

Data on the trajectories of various golf balls has been collected from a radar system [19]. We wish to correlate Reynolds numbers and spin numbers with lift and drag coefficients. The radar unit (ISG Trackman) used to obtain the trajectory data was placed near the initial position of the ball. Therefore, the second half of the trajectory - the descent - is far from the radar and the data is less accurate. It is in this region where the Reynolds number falls, and some interesting changes occur in the correlation between drag and lift coefficients, and the Reynolds and spin number. Our data indicates that around the apex of the balls trajectory a change in the flow regime over the ball may occur. It has also been noted that after the apex, the golf ball follows a path that seems to be almost parabolic (see Figure 1.2, indicating that aerodynamic effects are less important than gravitational effects in this region. We are interested in obtaining lift and drag coefficients for a wider range of spin and Reynolds numbers, as well as developing a better understanding of the flow around a golf ball in the post apex region.

It is well known that the spin of a golf ball affects the trajectory the ball takes after being struck by the head of a club. Davies [9] has derived the equations of motion for a golf ball subject to aerodynamic, gravitational and inertial forces. These forces may be represented by a set of differential equations.

$$
\begin{aligned}
& \frac{d^{2} x}{d t^{2}}=\frac{g F_{D}}{W} \cos \theta-\frac{g F_{L}}{W} \sin \theta \\
& \frac{d^{2} y}{d t^{2}}=-g-\frac{g F_{D}}{W} \sin \theta+\frac{g F_{L}}{W} \cos \theta
\end{aligned}
$$


where $g$ is the acceleration due to gravity, $F_{D}$ is the drag force, $F_{L}$ is the lift force, $W$ is the weight of the ball, and $\theta$ is the angle of launch relative to the ground. The lift and drag coefficients can be solved for explicitly as functions acceleration of the ball as follows.

$$
\begin{aligned}
& F_{L}=m a_{x} \sin \alpha+\left(m g+m a_{y}\right) \cos \alpha \\
& F_{D}=-m a_{x} \cos \alpha+\left(m g+m a_{y}\right) \sin \alpha
\end{aligned}
$$

When the lift and drag forces are set to zero, the equations describe a parabolic trajectory, as is expected for a body moving without aerodynamic effects:

$$
\begin{aligned}
& \frac{d^{2} x}{d t^{2}}=0 \\
& \frac{d^{2} y}{d t^{2}}=-g
\end{aligned}
$$

Near the beginning of the trajectory, the ball takes a path that is very nearly linear, indicating that the lift force is similar in magnitude to the force due to gravity. The path becomes more curved as the ball's spin and velocity decrease to drag. After passing through the vertical maximum of the trajectory, the ball's path is very nearly parabolic, indicating that aerodynamic effects are much smaller than the effect of gravity at this stage. We therefore explain this by saying the lift and drag coefficients are functions of the Reynolds number and the spin number. Just before the apex of the trajectory the ball moves slower than it did at the instant it was hit and the associated Reynolds number decreases. The lift and drag coefficients change according to their functional relationship to the Reynolds number and spin number. When the ball then begins to speed up again due to the 


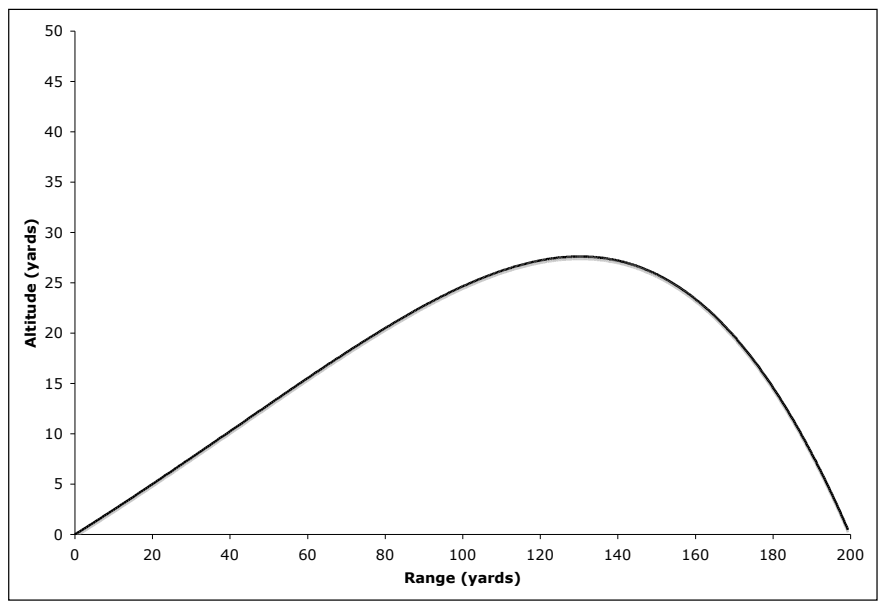

Figure 1.2: A graph of a typical golf ball's trajectory based on radar data. Note that the the $y$-axis is scaled to be four times larger than the $\mathrm{x}$-axis.

effect of gravity, the calculated lift and drag coefficients take on different values than they had previously in the trajectory, for the same speed. This implies that there is not a functional correspondence between the lift and drag coefficients and the Reynolds number. This is somewhat of a paradox because according to theory, the flight coefficients (lift and drag) must be functions of Reynolds number to successfully model flight. What we expect is that on a graph of drag coefficient versus Reynolds number, the path of the ball will move to the left (decreasing Reynolds number) as the ball slows down from its initial velocity after being launched from the tee.

We expect the drag coefficient to generally increase as the ball slows. This result is expected from examining the drag-Reynold's curve of any sphere (smooth, rough, or dimpled). After the ball reaches its highest altitude, it will soon after begin to accelerate again, due to gravity. As the ball speeds up, we expect the 


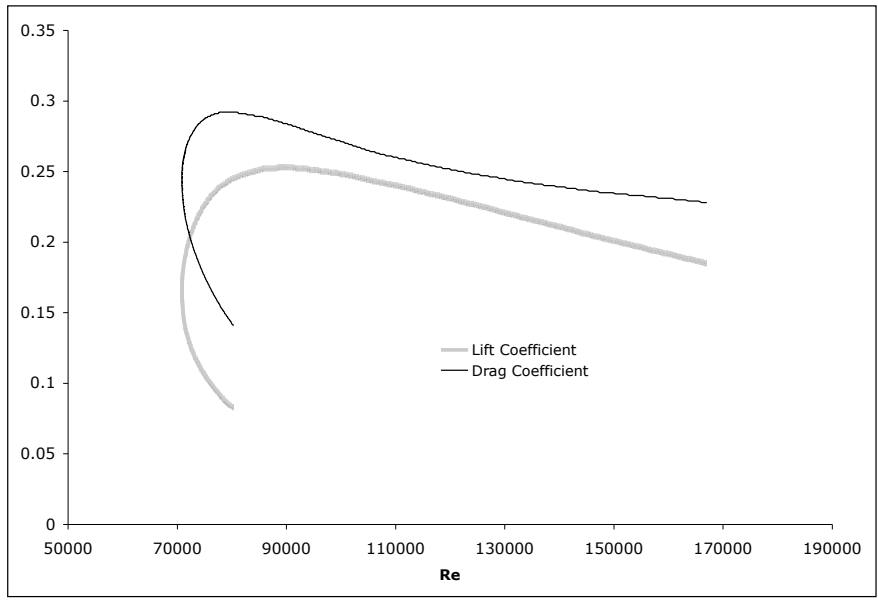

Figure 1.3: Flight coefficients vs Reynolds number. A graph of a typical golf ball's lift and drag coefficients vs Reynolds number. Based on radar data.

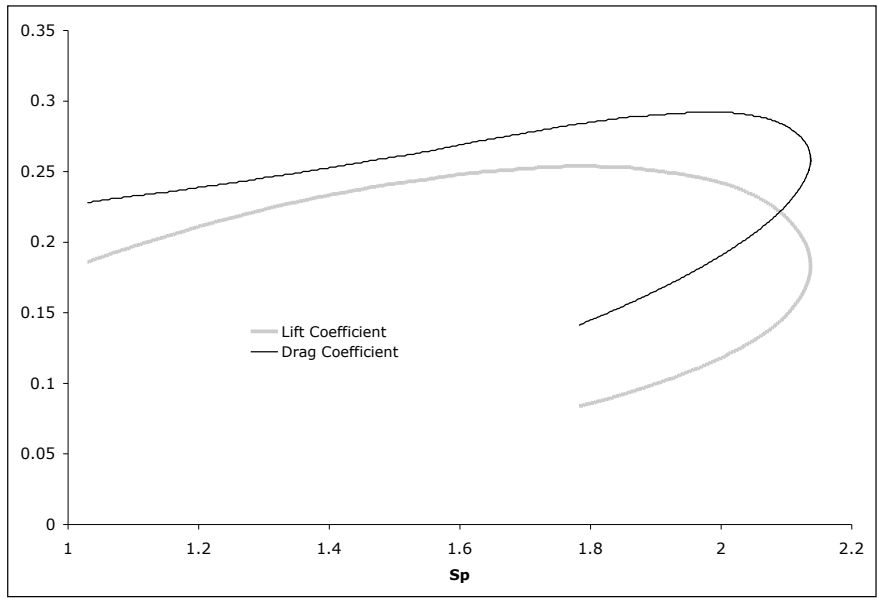

Figure 1.4: Flight coefficients vs spin number. A graph of a typical golf ball's lift and drag coefficients vs spin number. Based on radar data. 
curve on the drag-Reynold's graph to be "re-traced"; that is, for the aerodynamic state of the ball to move "left" until it starts to speed up again at which point the state will start to move to the right along the same curve. Our data shows, however, that as the ball begins to speed up its state does not follow the same curve as it was slowing. Instead, both the drag and lift coefficients continue to decrease as the ball accelerates. This finding has been confirmed independently by others who have studied golf ball trajectories [33]. Since the radar measures velocities, the numerical integrals and derivatives need to be calculated to obtain the position and acceleration of the ball, respectively. One method of obtaining the acceleration (to find the forces) is first-order difference method. This gives only a rough estimate, however, and a better method may be a slightly more complex equation. For example, the following finite difference approximation was used.

$$
\left.\frac{\partial u}{\partial x}\right)_{i, j}=\frac{3 u_{i, j}-4 u_{i-1, j}+u_{i-2, j}}{2 h}+O\left(h^{2}\right)
$$

where $u=u(x)$, and $h$ is the interval to be considered. This method leads to a smoother curve than the simpler approximation [31].

Currently, the largest source of trajectory data comes from the Indoor Test Range (ITR) at the United States Golf Association (USGA) Research and Test Center. The ITR uses light screens to determine the position of the ball with respect to time. From this information, the velocities, accelerations, etc. are easily obtained. The light screens are both vertical and angular, and are arranged over a 69 foot area. An iterative Newton-Raphson method on the equations of motion (see Eqs. 1.1, 1.2) is used to generate the required information. The nonfunctionality of the flight coefficients is also present in their data. It is typically 
truncated [27].

Once the trajectory data is available an empirical relationship is usually used to fit the data. The following functions are recommended by the USGA.

\begin{tabular}{|c|}
\hline $\ln (R e) /(R e)^{2}$ \\
\hline$e^{-R e}$ \\
\hline $1 / R e$ \\
\hline $1 /(R e)^{2}$ \\
\hline $1 /(R e)^{3}$ \\
\hline$R e$ \\
\hline$(R e \cdot S p)^{2}$ \\
\hline
\end{tabular}

Table 1.1: Independent functions suggested for curve fitting of golf balls' trajectories.

The USGA uses an algorithm to find the linear combination of these terms that yields the highest $R^{2}$-value. Quintavalla [26] used the following forms.

$$
\begin{aligned}
C_{L} & =\left[a_{1}+\frac{a_{2}}{R e^{5}}+\frac{a_{3}}{R e^{7}}\right]+\left[b_{1}+b_{2} \frac{\ln (R e)}{R e^{2}}+\frac{b_{3}}{R e^{2}}\right] \cdot \alpha \\
C_{D} & =\left[c_{1}+\frac{c_{2}}{R e^{3}}+\frac{c_{3}}{R e^{5}}+\frac{c_{4}}{R e^{7}}\right]+\left[d_{1}+d_{2} \frac{\ln (R e)}{R e^{2}} \cdot \alpha^{2}\right]
\end{aligned}
$$

Using his model, Quintavalla ran a numerical simulation to find the range of a drive differed from the test data by 0.3 meters [26]. These results are in good agreement with test data, but the equations are quite complex.

For our data, the golf balls were marked with reflective paint [18] and tracked using the radar system. Enough information was captured to accurately measure the spin rate of the ball throughout the first quarter of its flight. The data indicates that a ball's speed of rotation will decrease linearly with time. It is unclear from the literature what effect the initial speed of spin has on the ball. 
According to one study, a change in the initial spin speed of $\pm 50 \%$ will cause a change in the range of the ball of 1.7 yards [32].

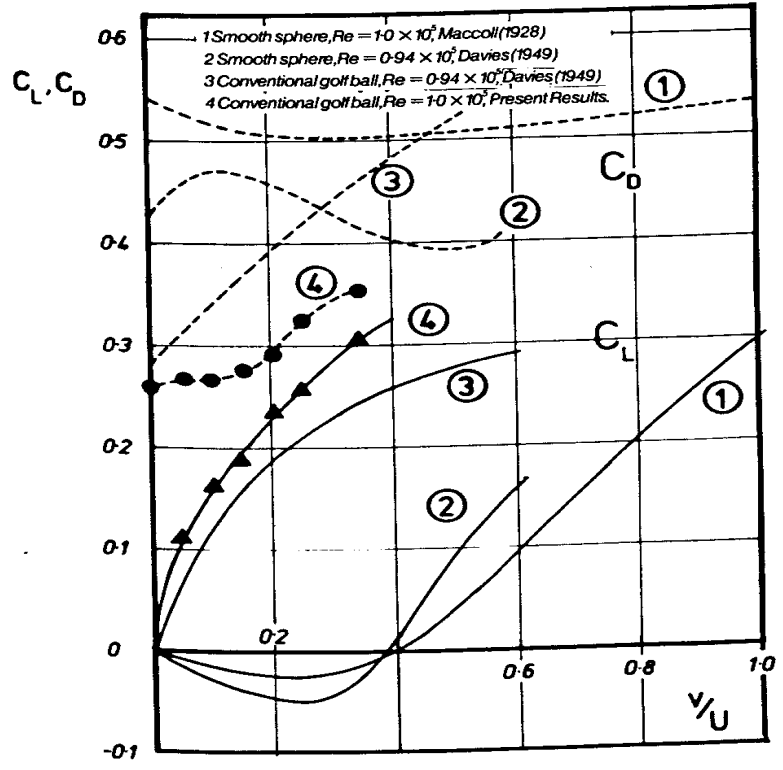

Figure 1.5: These results originally from Bearman and Harvey (1976) [5] show the discrepancy of the results of previous work performed by Davies [9].

\subsection{Background}

It was Sir Isaac Newton who first described a ball's trajectory being curved as a result of its spin. In 1672, he analyzed the motion of tennis balls, yet the same principles apply to golf balls today [9, 20]. Later, B. Robins published results on how a musket ball's spin produces the effect of a curved trajectory. Today it is known as the Magnus effect after the German engineer G. Magnus who described the lateral motion of cannon balls being fired from curved barrels which imparted spin onto the ball. A golf ball measuring, 1.68" in diameter, may spin at around 8,000 rpm during a drive [9]. This spin gives rise to a Magnus 
force that dramatically affects the trajectory of the ball. In fact, the Magnus force may be equal in magnitude to weight of the ball itself.

Magnus originally presumed that the force due to spinning was solely due to a pressure imbalance about the ball $[30,12]$. More modern theories suggest that this is only part of the explanation, and that boundary layer separation also plays an important role [20]. Magnus suggested that a ball rotating clockwise moving to the left, for example, would have air moving faster over its top side, and slower over its bottom side. From Bernoulli's equation (see Appendix B.1, we know that the faster air moving over the top of the ball has a lower pressure than the slower air underneath the ball. This gives rise to a lift force pushing the ball upwards. This analysis is not entirely incorrect, but it is incomplete. The faster air moving over the top of the ball has a lower relative velocity with respect to the ball surface than the air moving under the ball. The upper boundary layer undergoes a delayed separation while the lower boundary layer undergoes an early separation. This causes the wake of the ball to shift downward imparting some upward momentum to the ball. In the laminar regime, the separation points are around 90 degrees from the stagnation point. After the transition to turbulence, the points move back to around 120 degrees away from the stagnation point [2].

The functional relationship between the flight coefficients and spin have never been determined to a very accurate extent [5]. The results from both Bearman and Harvey (1976) and Davies (1949) both rely on the assumption of a constant lateral force during flight. These authors have employed the "ball-drop" method, where the spinning ball is dropped through the wind tunnel, and the deflection is measured. From this deflection, the average force on the ball is obtained. Only the assumption of constant force will allow the calculation of an instantaneous force. This assumption does not need to be made for the type of experiments 
presented in this paper. With the current system, the lateral force should be measurable.

Pressure taps have also been used to study golf balls in the past. The pressure distribution appears similar to a smooth sphere [1]. Aoki et al. (1998) even used a spark tracing method to visualize the separation points around a golf ball. It was found that the transition to turbulence occurs at roughly $70 \mathrm{ft} / \mathrm{s}(21.3 \mathrm{~m} / \mathrm{s})$, or at a Reynolds number of $6 \times 10^{4}$.

As for the theory used to analyze these types of flows, it is usually assumed that the gradients of flow variables (i.e. $\rho, \mathbf{U}$ ) are much smaller than the gradients of fluctuations (i.e. $\tilde{\rho}$, $\tilde{\mathbf{U}})[16]$. Mathematically, this means the Navier-Stokes equations,

$$
\begin{gathered}
\frac{\partial \mathbf{u}}{\partial t}+\mathbf{u} \cdot \nabla \mathbf{u}=-\frac{1}{\rho} \nabla p+\nu \nabla^{2} \mathbf{u} \\
\nabla \cdot \mathbf{u}=0
\end{gathered}
$$

may be simplified into a linear form. Note that in Eq. 1.10, $\mathbf{u}$ is the velocity vector, $p$ is the pressure, $\rho$ is the density, $\nu$ is the kinematic visocity, and $t$ is time. For linear stability, we decompose the motion into a mean part and a fluctuating part.

$$
\begin{aligned}
\mathbf{u}(x, y, z, t) & =\mathbf{U}(x, y, z)+\tilde{\mathbf{u}}(x, y, z, t) \\
p(x, y, z, t) & =P(x, y, z)+\tilde{p}(x, y, z, t)
\end{aligned}
$$

The variables in capital letters represent the mean velocity and pressure. These expressions derive from the assumption that the flow can be represented by a linear combination of a steady mean value and an instantaneous perturbation. Substituting Equations 1.12 and 1.13 into Eq. 1.10 produces the linearized per- 
turbation equations.

$$
\begin{gathered}
\frac{\partial \tilde{\mathbf{u}}}{\partial t}+\mathbf{U} \cdot \nabla \tilde{\mathbf{u}}+\tilde{\mathbf{u}} \cdot \nabla \mathbf{U}=-\nabla \tilde{p}+\frac{1}{R e} \nabla^{2} \tilde{\mathbf{u}} \\
\nabla \cdot \tilde{\mathbf{u}}=0
\end{gathered}
$$

where Re is, of course, the Reynolds number. In order to understand exactly what is happening with the flow around a golf ball, a knowledge of what causes turbulence is needed. Although a good deal is known on the subject, the study of turbulence continues to evolve. Fundamental questions remain as to how turbulence should even be described [14]. The linearized perturbation equations are useful because they do a good job of predicting flow conditions in this regime [17], yet are simple enough to be of use in numerical simulations. They therefore provide some (numerically) testable predictions for the Navier-Stokes equations. These equations main use is in modeling the stability of boundary layers, which is crucial to understanding the flow around a golf ball.

One of the main uses of these equations is in deriving an ordinary differential equation known as the Orr-Sommerfield equation. In a non-dimensional form, it is

$$
\frac{1}{i \beta R e}\left(\frac{d^{2}}{d z^{2}}-\beta^{2}\right)^{2} \phi=(U-c)\left(\frac{d^{2}}{d z^{2}}-\beta^{2}\right) \phi-U^{\prime \prime} \phi
$$

where $\beta$ is a constant, $U(z)$ is the velocity profile, $\phi$ is a stream function, and $c$ is the wave speed in the fluid. This equation is derived for Poiseuille flow, then expanded for other boundary conditions. In general, the solutions are found numerically for all but very simple circumstances. By studying solutions for basic problems, something can be learned qualitatively about more complex flows such as around a sphere. From applying the equation to Poiseuille flow, it can be calculated that the boundary layer becomes unstable at around $R e=5,800$. Although it is not easy to make a similar calculation for a sphere, it does seem 
that there will be a point on the sphere where the boundary layer will become unstable and separate [23].

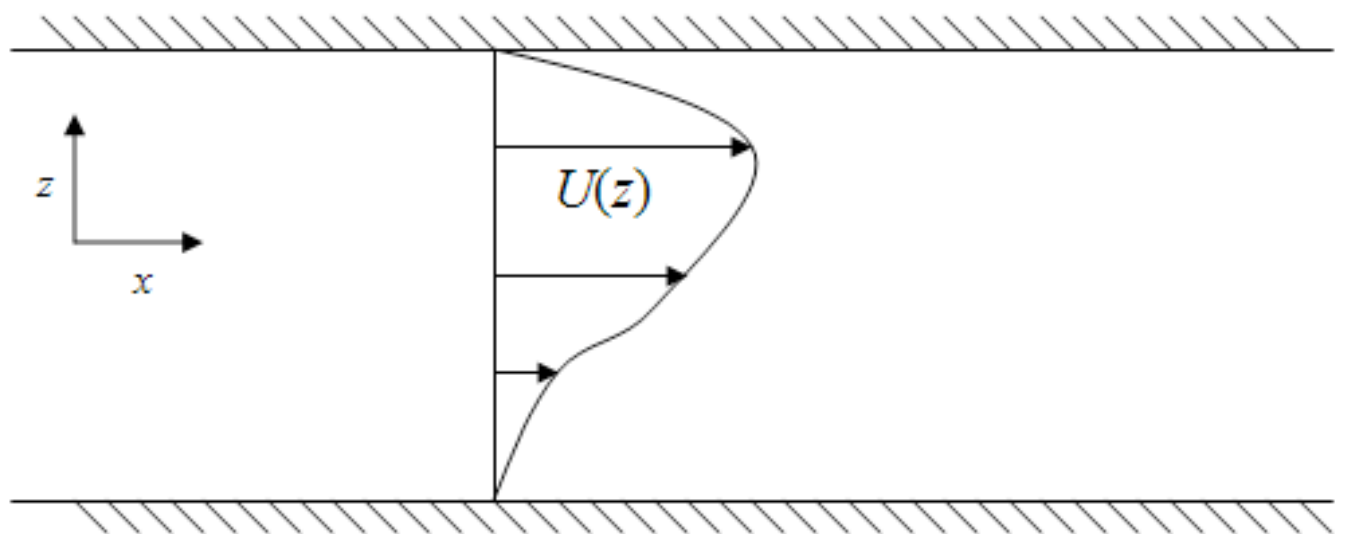

Figure 1.6: Diagram of the velocity profile of a perturbed Poiseuille flow illustrating instability [22].

The Orr-Sommerfeld equation suggests that certain velocity profiles are unstable and that boundary layers will naturally separate from the object in the flow. This is important because it provides insight into separation occurs at all, and that it is a natural part of the mechanics of fluid flow. Also, it is assumed that the perturbation velocity has wave-like characteristics, consistent with observation. Strictly speaking, the boundary layer separation and wave-like characteristics of the flow do not constitute turbulence. However, stability and turbulence are intimately related, and there is great interest in experimentation into the nature of turbulent boundary layers [28]. Any calculations made using this equation should be testable given suitable equipment. Therefore, the development of new testing procedures and apparatus is important for research in the area of fluid mechanics. 


\section{Chapter 2}

\section{Experimental Apparatus}

The test equipment used to conduct the experiments was composed of a wind tunnel, a fixture, and a data acquisition system (DAQ) at the Cal Poly Mechanical Engineering Department. In order to observe the effects of a translating and rotating golf ball under laboratory conditions, an apparatus was created to spin the golf ball within a wind tunnel. The system presented here is original, in that all other wind tunnel work on golf balls have used different techniques (see Chapter 1. This system was created with the intent of being the first device to spin a golf ball in the wind tunnel using a solid axle and force transducers.

\subsection{Wind Tunnel}

The Department of Mechanical Engineering owns and operates a wind tunnel primarily used for education (Fig. 2.1). Much of the research conducted for this thesis hinged on the operation of the wind tunnel. The wind tunnel is of the open-circuit type and is manufactured and assembled by ELD, Inc. model 406(B). The advantage of using an open-circuit wind tunnel is more uniformity 
of the flow and less free-stream turbulence [25]. The alternative, a closed-circuit tunnel, will propagate disturbances from the fan (located after the test section) around the loop back into the test section. The prime mover is a fan connected to a variable frequency drive and three phase motor. The drive frequency may be varied from $0.0-60.0 \mathrm{~Hz}$ in $0.1 \mathrm{~Hz}$ increments [15]. At low speeds the wind tunnel contributes minimum noise to the system. At higher speeds, however, some large amplitude vibrations develop which generally increases the spread of data taken by the force transducers.

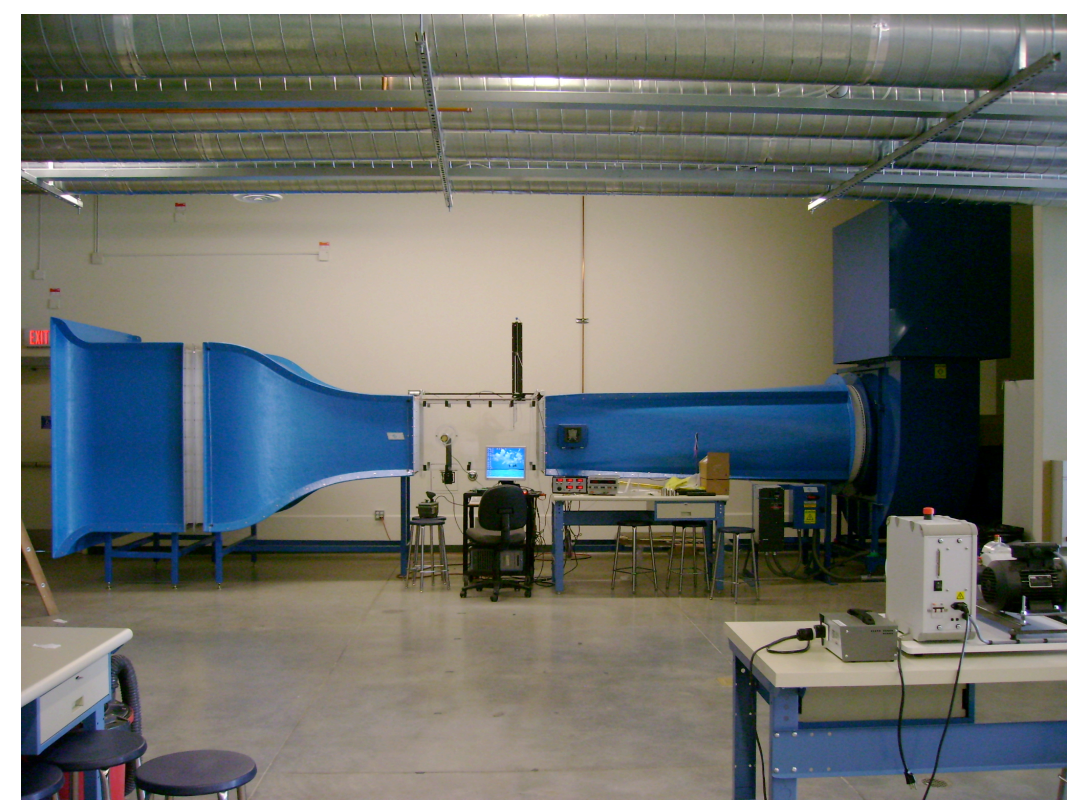

Figure 2.1: The wind tunnel used for testing.

The velocity profile of the flow in the tunnel [29] was ascertained by measuring the pressure variation in the z-direction (vertical direction). The given profile was measured along an axis running through the center of the test section, at 60 $\mathrm{Hz}$, just before the end of the test section (Fig. 2.2). The pressure and therefore velocity distribution is relatively uniform throughout, with a very small boundary layer on the bottom surface and a larger one on the upper surface. The large 


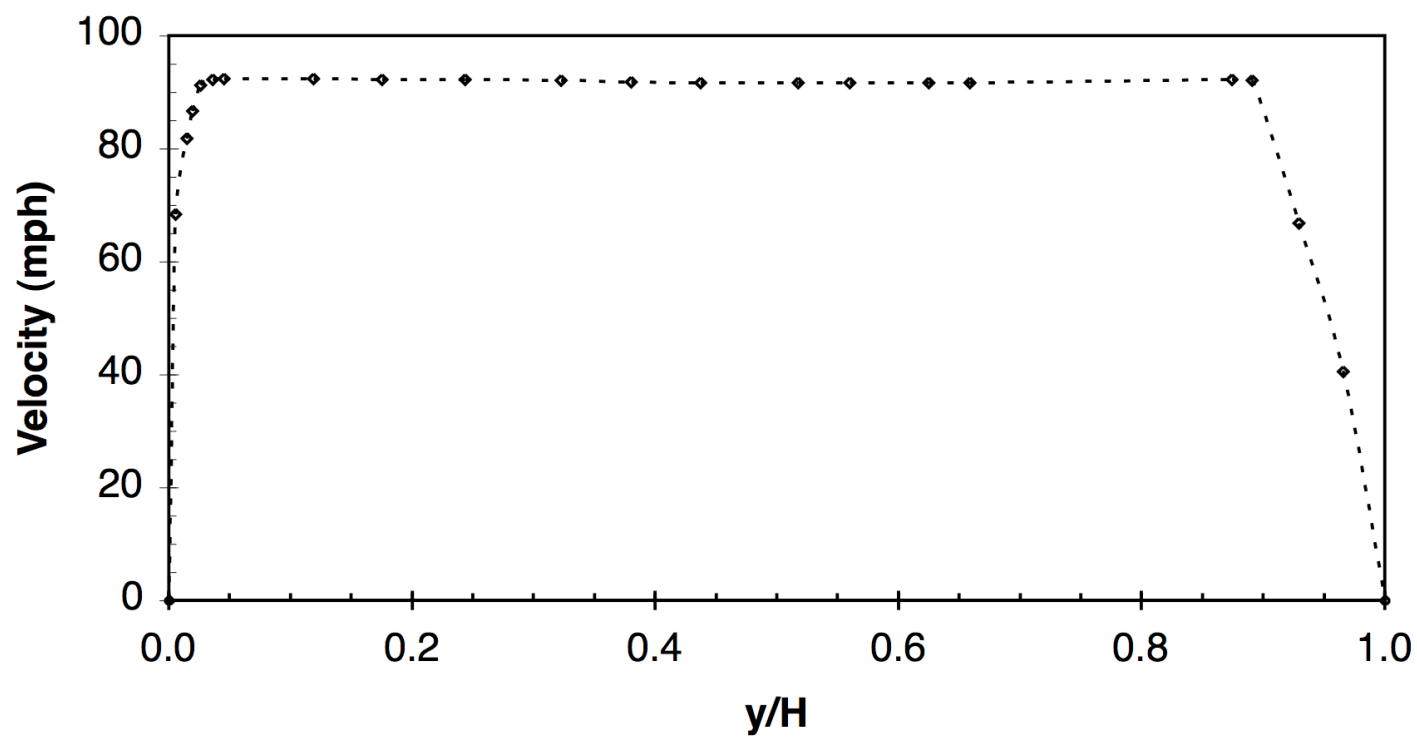

Figure 2.2: Graph of air speed versus normalized height of test section.

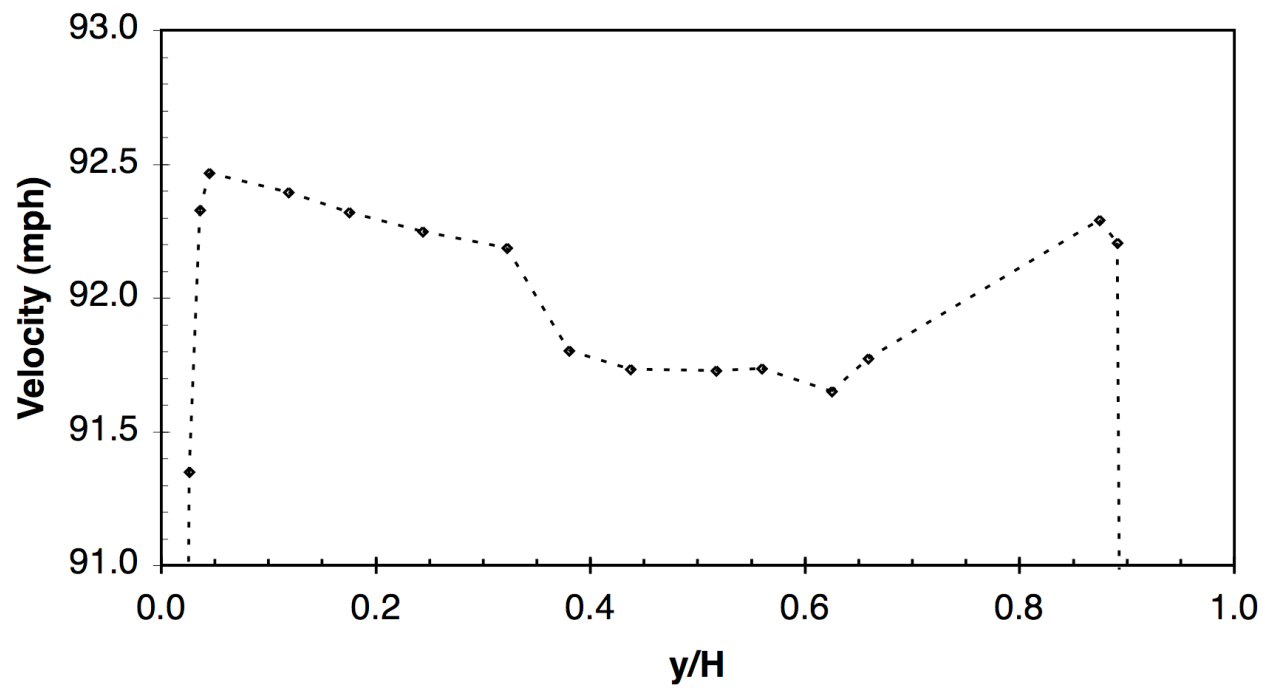

Figure 2.3: Graph of air speed versus normalized height of test section emphasizing that the total variation is just $1 \mathrm{mph}$. 
upper boundary layer is due to a slit which the pitot tube probe enters the tunnel from its mount outside the tunnel. On closer inspection, the velocity profile for the bulk of the cross sectional area shows a dip near the central area. The total variation of the air speed is $1.0 \%$ of the average speed. Noting that the the only deviation from a very flat profile is due to the slit on the upper surface of the wind tunnel, it is assumed that the transverse (y-direction) velocity profile should look very flat with a small boundary layer on either side wall. With this assumption, it is concluded that the same test results should be obtained whether the model is placed at the geometric center of the cross section, or whether it is placed, say, halfway between a side wall and the center in the transverse direction. This is exactly what was done for the test setup.

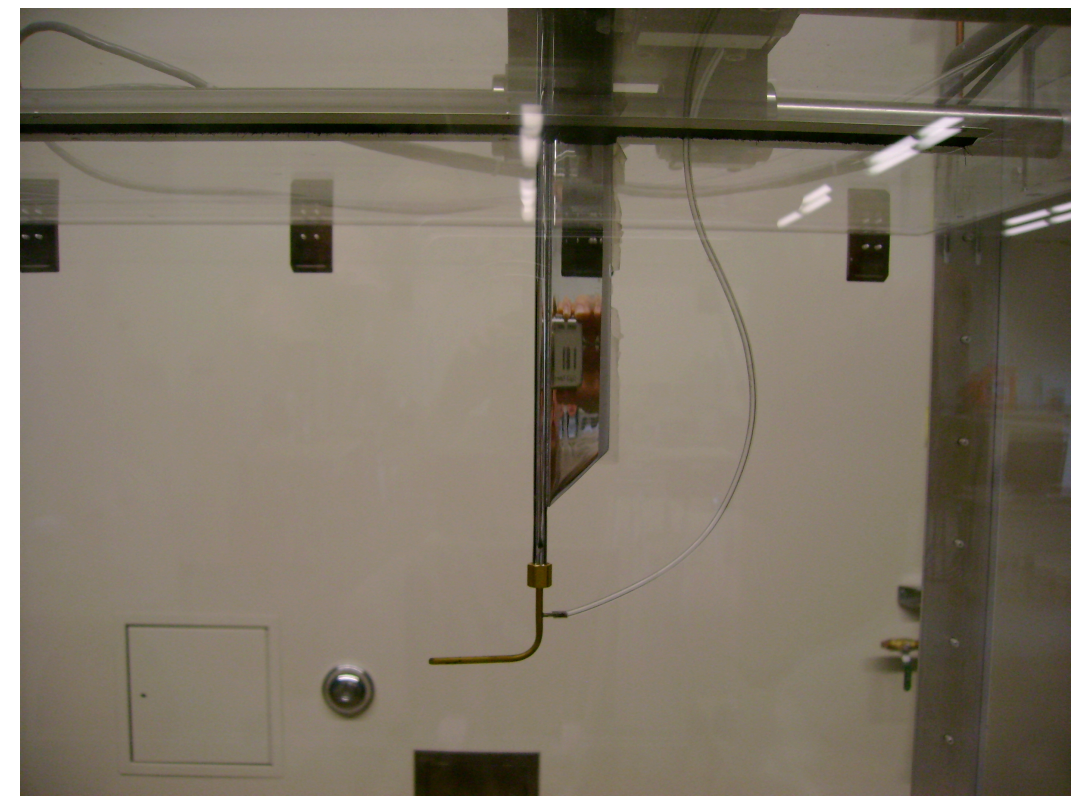

Figure 2.4: Photograph of the Pitot tube used to obtain pressure measurements of the free stream flow. 


\subsection{Sting}

The ball spinning fixture (described below) was attached to a steel bar that protrudes into the test section from the underside of the wind tunnel known as a sting. It is surrounded by a fairing (shroud to reduce drag) in the shape of an airfoil that protects the sting from the freestream air. The sting connects to a force transduction housing mounted to the underside of the wind tunnel. The housing is made of aluminum and uses two linear variable differential transformers (LVDT), one for drag and one for lift.

LVDTs work by running an AC current through a primary coil which circumscribes a ferromagnetic core attached to the object to be measured. As the core moves, a voltage is induced in the two secondary coils set on either side of the primary coil. This voltage is linearly dependent on the position of the core. A diagram of an LVDT appears in Figure 2.5. The LVDT that determines the lift force is connected directly to the base plate. The drag LVDT is actually supported in an inner carriage making it less susceptible to vibration from the fan motor.

The sting itself is a steel bar with one hole containing $1 / 4 "-20$ thread. A fairing (aerodynamic shroud) protects the sting from registering a force due to the free-stream air. For more information on the fairing, see sections 4.4.1 and 5.4. The sting only reads the forces in the $\mathrm{x}$ - and z-directions. In order to measure a moment, two stings are generally needed [25]. In order to ensure that the lift and drag are actually measured separately, a series of test were done. Based on these results it was concluded that the two measurement channels were completely independent.

An ideal fairing will act as an aerodynamic shroud, shielding the sting from the 


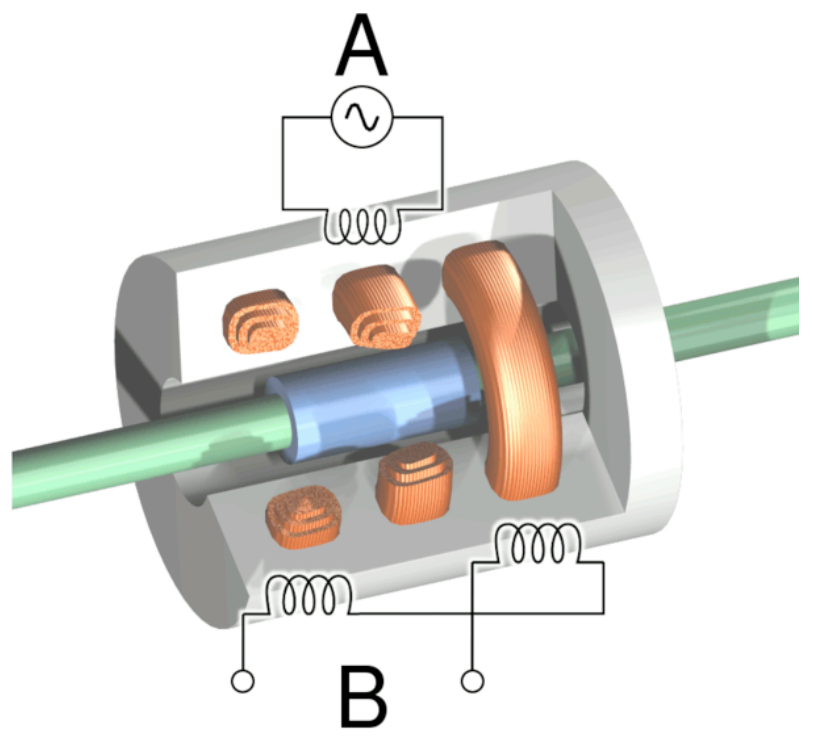

Figure 2.5: A cutaway drawing of an LVDT showing the primary and secondary coils and the ferromagnetic core [24].

free-stream air. However, in our case, there was significant interaction between the fairing and sting. Although the fairing never contacts the sting, it will still transmit some force. Presumably, forces acting on the fairing deform the platform to which all other force measuring apparatus are attached, causing a false force reading. A force imparted on the fairing in the positive drag direction causes a positive lift reading; a force imparted in the positive lift direction also causes a positive lift reading.

\section{$2.3 \quad$ Fixture}

The apparatus that actually holds and spins the ball will be referred to as the fixture. The fixture is of custom design and the main development of the thesis. It is composed of a shaft supported at both ends by bearings. On one 


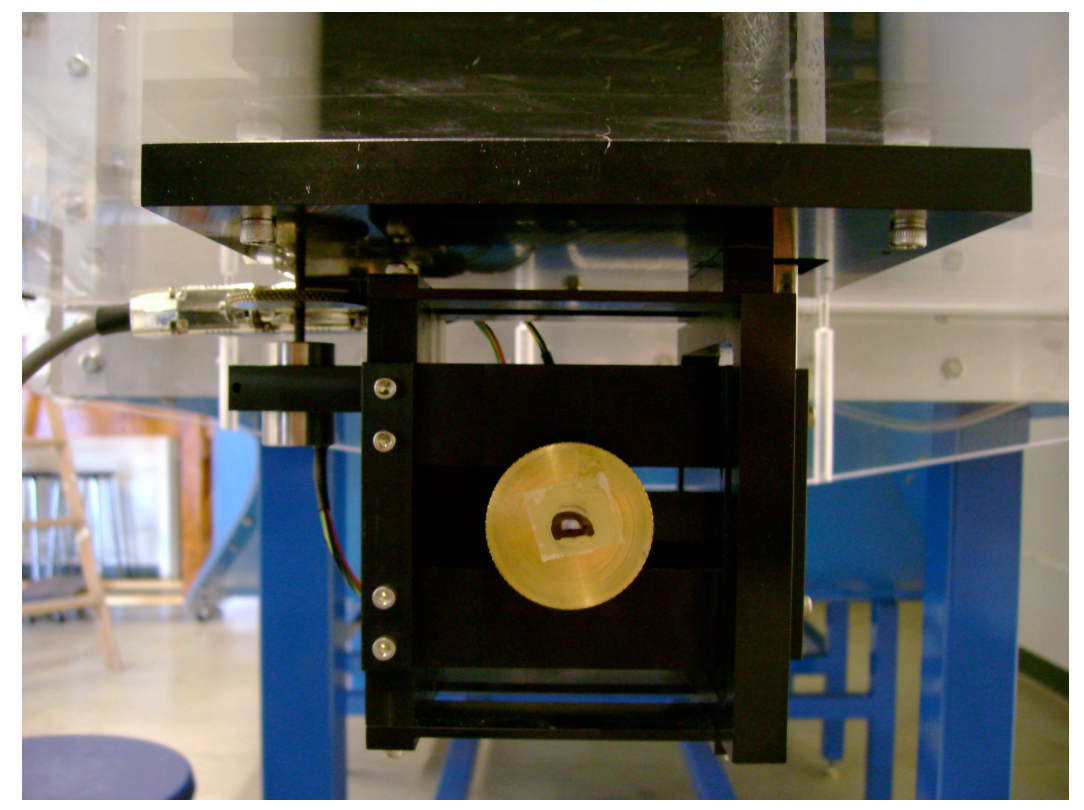

Figure 2.6: A photograph of the drag calibration dial of the sting fixture. It is marked with a "D" for drag.

side the bearing was housed in a piece connected to the sting, and on the other, the bearing was pressed into a plate fit into the side wall of the wind tunnel.

The choice of bearings and couplings was determined by testing various setups and determining which gives the lowest amplitude of vibration for the entire spectrum of testing which ranges from 1,000 rpm to 8,600 rpm. The types of bearings tried were ball bearings, plastic and brass collar bearings, and ball joint swivel (BJS) bearings. The types of couplings tried were flexible with solid spider, helical beam, high-offset slotted-disc, and rigid.

The ball may be spun up to a maximum rotational speed of 8,600 rpm $(\omega=900 \mathrm{rad} / \mathrm{s})$ given the gear ratio used in the transmission. The transmission consists of a pair of nylon gears of ratio $3.25: 1$. The large gear was attached to the motor shaft by a cyanoacrylate adhesive. The small gear was also attached by a cyanoacrylate to the small gear which in turn is pressed through a bear- 


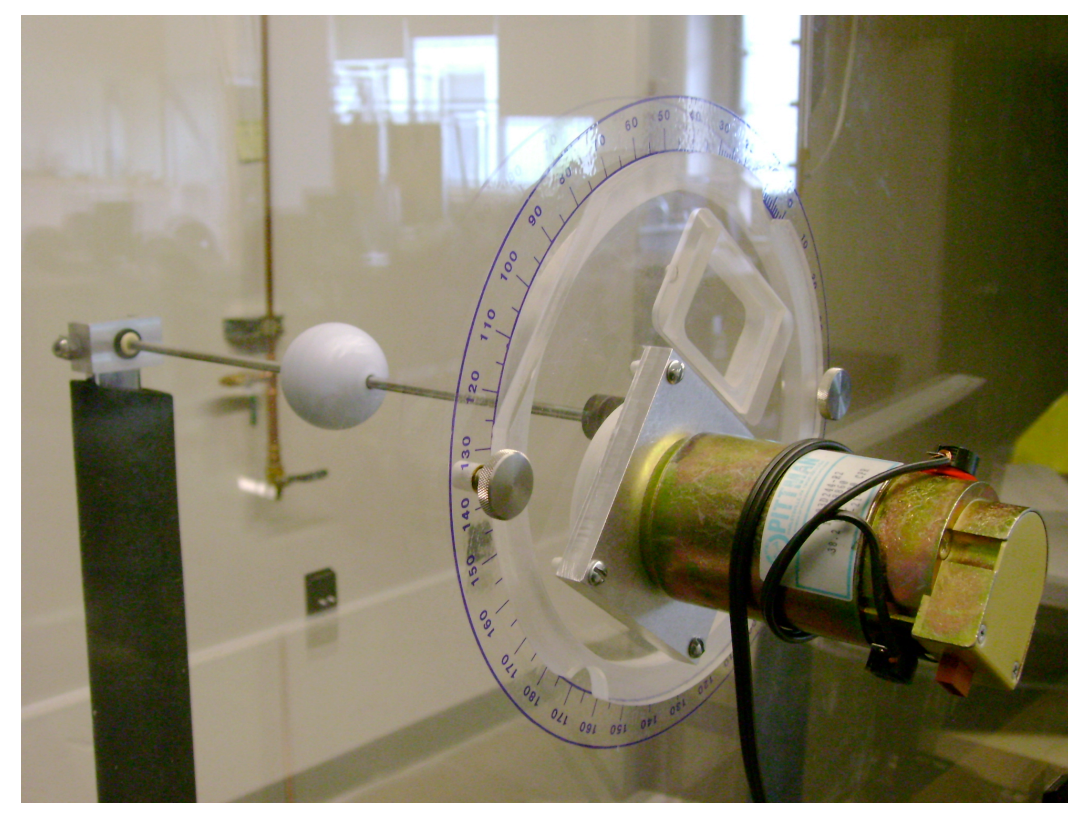

Figure 2.7: The sting can be seen on the left and the motor on the right. The ball is being spun on the shaft in the center.

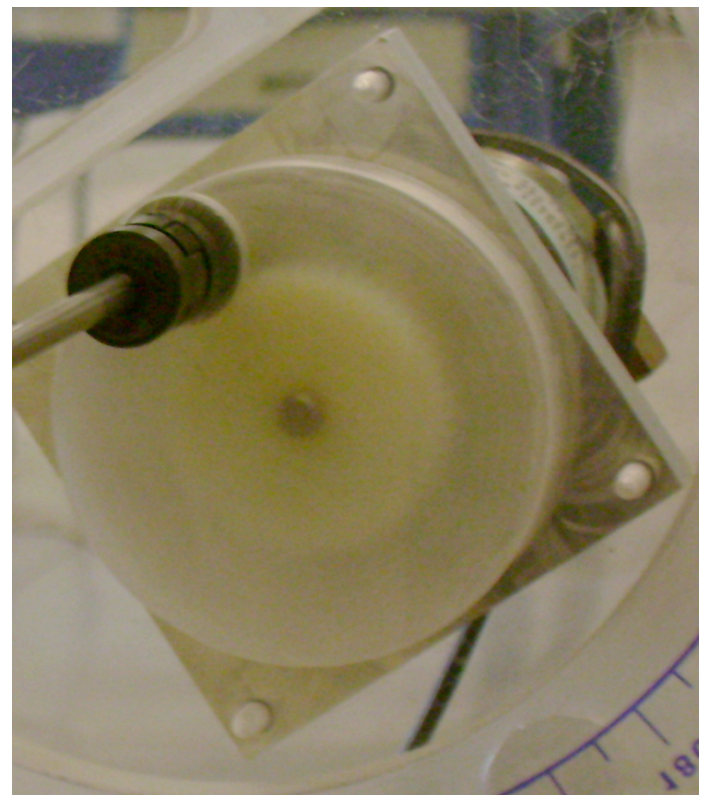

Figure 2.8: Photograph of the drive gear seen through the clear gearbox. 
ing. Initially, a different design that supported the ball in between two posts was going to be used. This design, shown in Appendix A.2, had the motor inside the wind tunnel. Some advantages of this design include a shorter and better supported axle and no contact with the wind tunnel side wall. The drawbacks include a large cross sectional area, the possibility of large amplitude vibration, and supplying power to the motor. After a full 3-D model was constructed, it was decided that a new design was necessary.

The final design houses the motor outside the test section, and solves the problem of a long rotational shaft by the use of appropriate bearings and couplings. The combination that proves most stable is to use a spider bearing at the motor-output side of the shaft, and a plane plastic bearing at the sting end of the shaft. Through experimentation, it was discovered that the most stable configurations of the shaft were ones that used a flexible coupling on the motor-out side and a rigid bearing system on the sting side. Many of the parts were purchased off the shelf (McMaster-Carr), in which case a part number is listed in the bill of materials, presented in Table 2.1 .

As mentioned in the previous section, the ball was placed halfway between the side wall and the center of the wind tunnel. This distance was six inches. Since the ball was not directly attached to the sting, there was a transmitted force and moment from the ball to the force transducer that needed to be accounted for. A simple model was constructed and is shown in figure 2.9

The model assumes the forces imparted on the ball act on the center of the shaft as a concentrated load halfway between the supports. At one end, the shaft was attached to a flexible coupling, which permited some rotation provided that it was small. At the other end, the shaft sat in a plastic bearing that was free to rotate. Therefore, the model used in the thesis assumes that the shaft was 


\begin{tabular}{|c|l|c|c|c|}
\hline No. & Description & Part Number & Material & Quantity \\
\hline \hline 1. & Bearing Housing & - & Aluminum & 1 \\
\hline 2. & $\begin{array}{l}\text { Ball Joint Swivel Bear- } \\
\text { ing }\end{array}$ & $1071 \mathrm{~K} 11$ & Nylon Composite & 1 \\
\hline 3. & $\begin{array}{l}1 / 4-20 \text { Standard Hex } \\
\text { Cap Screw }\end{array}$ & $92865 \mathrm{~A} 546$ & Steel & 1 \\
\hline 4. & $1 / 4-20$ Acorn Nut & $91875 \mathrm{~A} 130$ & Steel & 1 \\
\hline 5. & $1 / 4 "$ Washer & $95647 \mathrm{~A} 125$ & Delrin & 4 \\
\hline 6. & Precision Shaft & $1327 \mathrm{~K} 53$ & Steel & 1 \\
\hline 7. & $\begin{array}{l}\text { 3/16" Flexible Spider } \\
\text { Shaft Coupling }\end{array}$ & $9845 \mathrm{~T} 1$ & Aluminum & 2 \\
\hline 8. & Spider & $9845 \mathrm{~T} 11$ & Urethane & 1 \\
\hline 9. & Short Shaft & - & Steel & 2 \\
\hline 10. & Small Gear & $57655 \mathrm{~K} 34$ & Nylon & 1 \\
\hline 11. & Large Gear & $57655 \mathrm{~K} 48$ & Nylon & 1 \\
\hline 12. & 38.2 VDC Motor & - & - & 1 \\
\hline 13. & Motor Mount & - & Acrylic & 1 \\
\hline 14. & Plug & - & Acrylic & 1 \\
\hline 15. & 8-32 Machine Screws & - & Stainless Steel & 8 \\
\hline
\end{tabular}

Table 2.1: Bill of materials for the final design of the test apparatus.

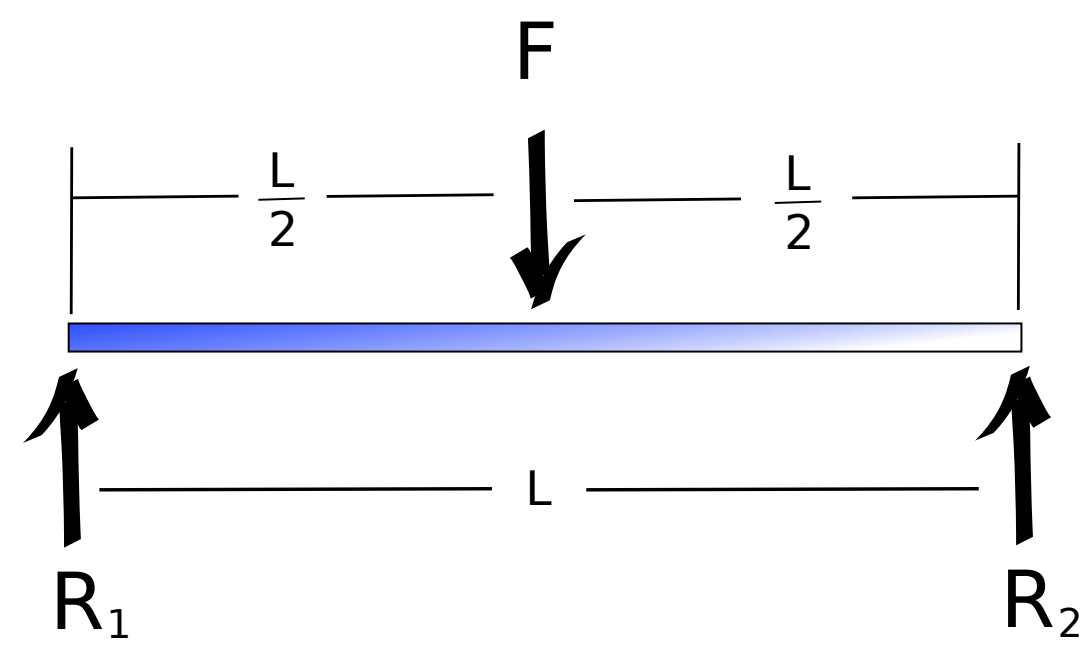

Figure 2.9: Diagram of force balance on the axle of rotation. 
simply supported. By summing the forces acting on the shaft, a set of equations was obtained.

$$
\begin{aligned}
& F=R_{1}+R_{2} \\
& \frac{F l}{2}-R_{2} l=0 \\
& F=2 R_{2} \\
& 2 R_{2}-R_{2}=R_{1}
\end{aligned}
$$

After combining equations we have the desired relationship.

$$
F=2 R_{2}=2 R_{1}
$$

So, the force read by the force transducer $\left(R_{1}\right)$ was half that of the aerodynamic force on the ball. The DAQ was programed to incorporate this factor.

\subsection{Data Acquisition System}

National Instruments LabVIEW software version 8.0 was utilized for data acquisition (details provided in App. C. The flow of data can be described as follows. The strain gages transduce the aerodynamic forces on the model into an voltage amplitude modulation direct current signal. The signal is fed into the receiver where values are amplified. Connected to the receiver is a National Instruments PCI card (NI PCI-6036E). The PCI card reads the analog signal with an amplitude range of $\pm 10 \mathrm{~V}$. A custom LabVIEW program then reads and writes the data to a spreadsheet file where it can be analyzed. 
The LabVIEW program was used to collect data at rates from $50 \mathrm{~Hz}$ to 20, $000 \mathrm{~Hz}$. Different frequencies were used under the same conditions in order to verify that the sampling frequency did not affect the reading. Testing verified that similar results were obtained on all channels regardless of the sampling frequency. Also, channels other than 0,1 , and 2 were tested to verify that the DAQ channels actually utilized for data processing were not unique in any way.

Data was taken at up to 20,000 points per second so that the sampling frequency was over twice the rotational frequency of the ball at its maximum speed. This is important so that the force due to an eccentric load on the rotating shaft can be measured and accounted for. Using a Haas CNC lathe to drill the center hole in the golf ball ensures that the rotational axis of the ball very nearly passes through the center of mass. The noise level of the signal is well measured by the standard deviation of the mean. The standard deviation for the signal at zero velocity and zero spin is $0.005 \mathrm{~V}$. The standard deviation for zero velocity and a spin of 8,600 rpm is $0.029 \mathrm{~V}$. Therefore, the added noise that comes from spinning the ball is $0.024 \mathrm{~V}$. This number is quite small, and represents an acceptable level of noise in the signal. A different trend is evident at higher wind speeds, however. Averaging several readings, a non-rotating ball operating at the maximum speed the wind tunnel allows, $\sim 160 \mathrm{ft} / \mathrm{s}(\sim 48.8 \mathrm{~m} / \mathrm{s})$, gives a standard deviation of $0.204 \mathrm{~V}$. Interestingly, when the ball is spun at the maximum spin rate allowable with the current system $(\sim 8,600 \mathrm{rpm})$, the standard deviation has been measured at $0.181 \mathrm{~V}$. It is unclear whether the spinning of the ball has a sort of stabilizing effect, but it seems that a rotation of the ball does not add any statistically significant noise to the system.

It is worth noting that the highest rotational speed was not associated with the highest standard deviation of results. In fact, there were some natural fre- 


\begin{tabular}{|c|c|c|}
\hline Wind Velocity & Spin Velocity & Standard Deviation \\
\hline \hline $0 \mathrm{ft} / \mathrm{s}$ & $0 \mathrm{rpm}$ & 0.005 \\
\hline $0 \mathrm{ft} / \mathrm{s}$ & $8500 \mathrm{rpm}$ & 0.029 \\
\hline $160 \mathrm{ft} / \mathrm{s}(48.8 \mathrm{~m} / \mathrm{s})$ & $8500 \mathrm{rpm}$ & 0.181 \\
\hline $160 \mathrm{ft} / \mathrm{s}(48.8 \mathrm{~m} / \mathrm{s})$ & $0 \mathrm{rpm}$ & 0.204 \\
\hline
\end{tabular}

Table 2.2: Comparison of standard deviations for some typical conditions.

quencies encountered during testing. The largest amplitude vibrations occurred at around 4,000 rpm. The actual speed of highest amplitude was not, in general, repeatable. This indicates that the speed of highest amplitude might be a function of temperature, humidity, or some other uncontrolled variable. It is thought that the bearing-shaft interaction, which is thought to be responsible for much of the instability of the rotation, is highly dependent on these variables of weather.

The National Instruments CB-68LP board was used to transfer the signal to the computer. The board is recognized by the program using National Instruments software, the correct board was picked from a drop-down menu so that LabVIEW may configure itself accordingly. There is a diagram which visually displays the correspondence between pins on the breadboard and channels on the PCI. The channels may then separately named and used in a LabVIEW program.

The rotational speed of the ball was measured using a strobe tachometer called the Strobotac, type 1531, General Radio Company. The Strobotac can measure speeds between $100 \mathrm{rpm}$ to $25,000 \mathrm{rpm}$. It has not been explicitly calibrated prior to being used, but a different Strobotac of the same model type was used to verify the results of the first. 


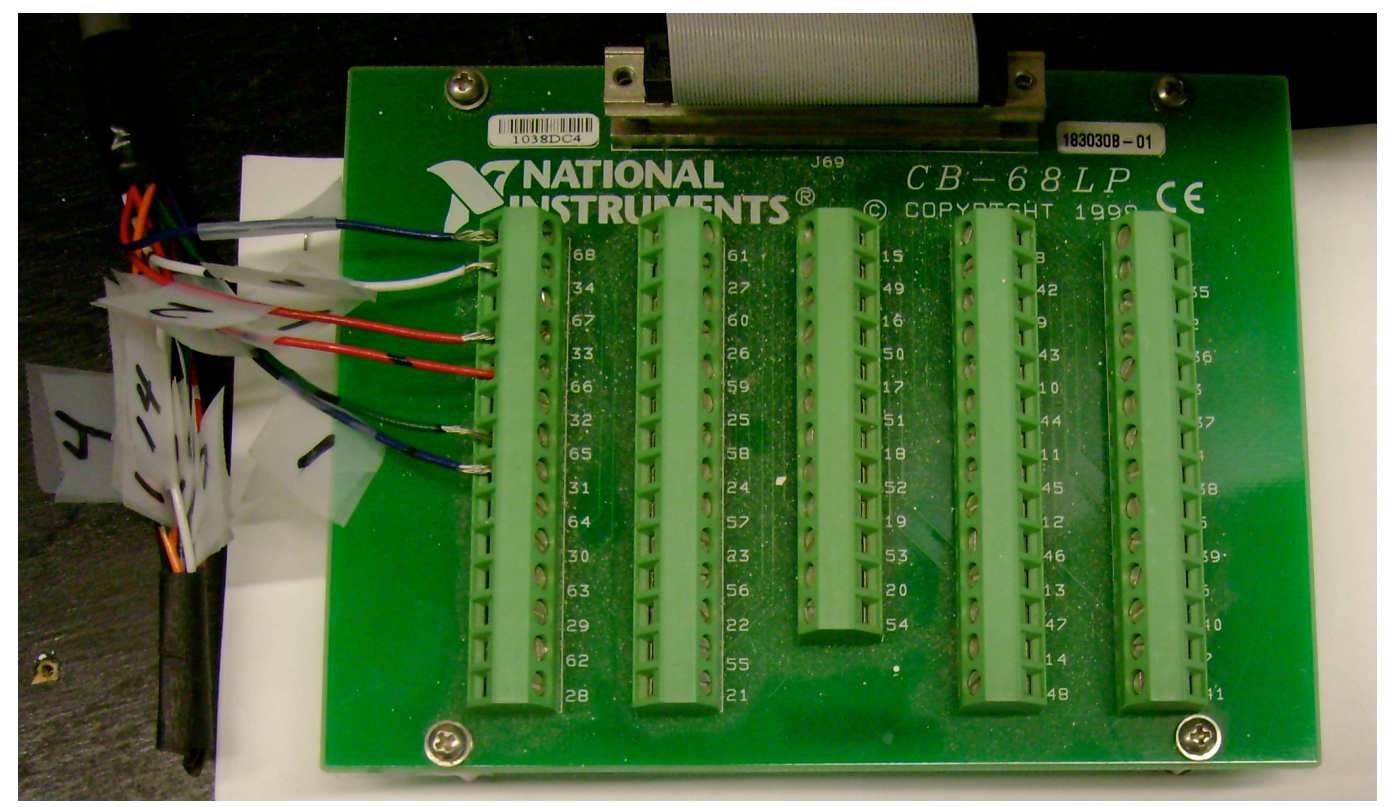

Figure 2.10: The CB-68LP breadboard was used to link the signal from the output box to the computer.

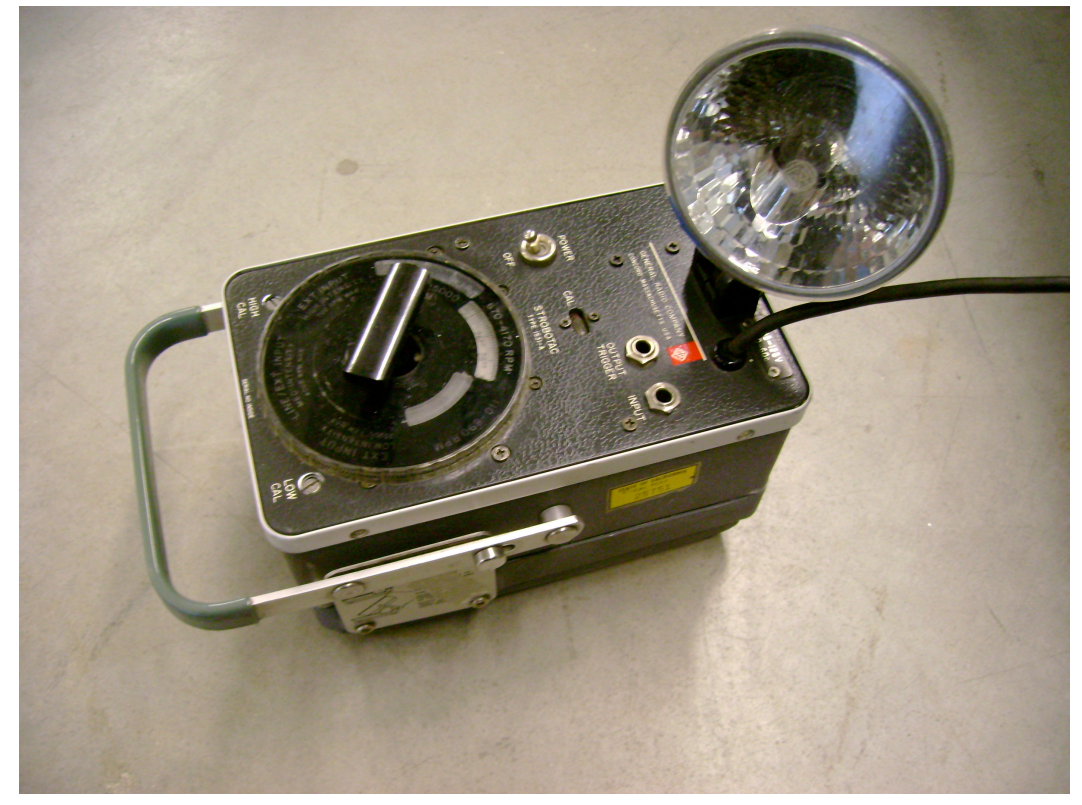

Figure 2.11: The Strobotac was used to measure the spin speed of the golf ball. 


\section{Chapter 3}

\section{Experimental Procedure}

The calibration of the force transducers was done using weights of $100 \mathrm{~g}, 200 \mathrm{~g}$, $500 \mathrm{~g}$, and $1000 \mathrm{~g}$. It should be noted that the calibration was performed using values from the DAQ program and not the display box that directly attaches to the LVDTs via a cable. The DAQ reads different values and, in particular, different calibration slopes. Also, time averaging was used to get a much more accurate reading than can be obtained by reading off values from the display box alone. During calibration, the weights were placed in random order on the sting in the drag direction and the lift direction. A clamp was used to minimize the possibility of movement during calibration. See Figure 3.1.

The calibration readings were graphed and fitted to a linear curve using Microsoft Excel. The results were very repeatable, and the lift and $\operatorname{drag} R^{2}$ values were found to be greater than $R^{2}=0.9999$ in both cases. The calibration curves are presented in Appendix D.

While recording data, two LabVIEW programs ran simultaneously. The first program, "recording program," reads, manipulates, and writes data. The sec- 


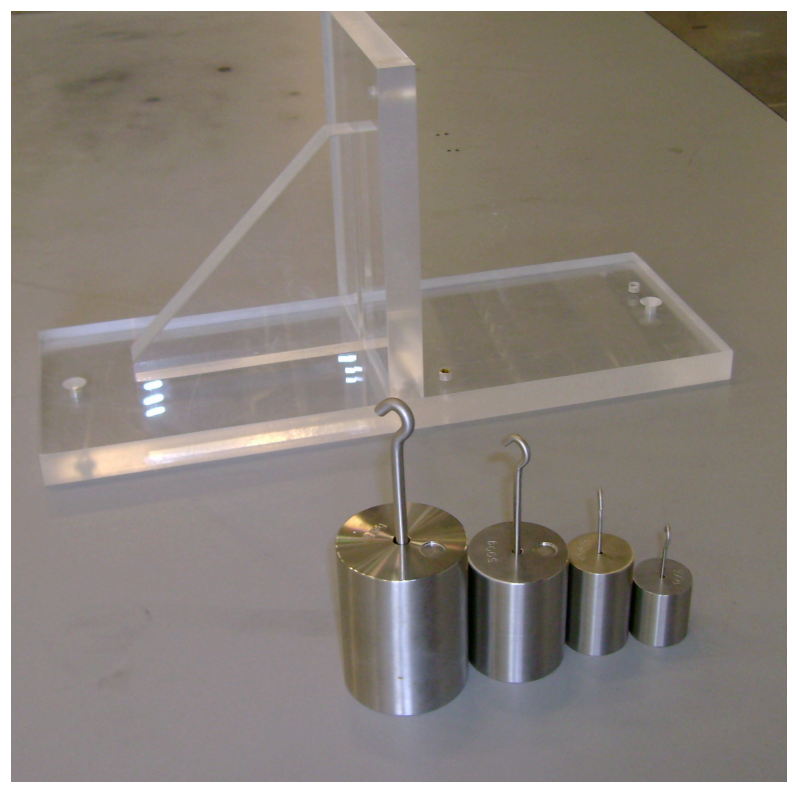

Figure 3.1: Calibration Equipment. The fixture was used to hold the sting unit secure while the weights were hung from it.

ond program, "streaming program," displays instantaneous values on a graph. The recording program samples data from three channels (see Table 3.1) and displays the title "Wind Tunnel DAQ." Ambient conditions such as temperature, humidity, and pressure were not recorded by the the DAQ. For screenshots of the programs mentioned in this section, see Appendix C.

\begin{tabular}{|c|c|}
\hline Channel & Data Stream \\
\hline \hline 0 & Drag \\
\hline 1 & Lift \\
\hline 2 & Pressure \\
\hline
\end{tabular}

Table 3.1: Input Channels.

The sampling rate was set by opening the National Instruments hardware control panel, and entering the desired frequency. The number of samples taken is specified in the recording program. Once these options were set, a selection needed to be made to tell the program whether to write a new file or append to 
an existing file. If the former option was chosen, the user is prompted for a file name. If the latter option was chosen, the filename must already be selected in the appropriate box appearing in the interface. Another feature of this program was a button displaying the text "Open Streaming Data?" If this option was selected the recording program would automatically open the streaming program after writing the first set of data to a file.

When the "run" button was clicked, the program began taking data. This was a manual trigger. To calculate how long the program would run, the number of samples to be taken was simply divided by the sampling frequency. After the data was taken, it was written to a file in the directory specified by the user. The program was immediately ready for another round of data collection. The data was collected via a subroutine called "Analog 2D DBL NChan NSamp." A 2D array of floating point numbers was outputted. The array was then broken up into three one-dimensional arrays corresponding to drag, lift, and pressure information. Next, each array was read by another subroutine that calculates the mean and standard deviation. The mean was not a running average, rather, the entire sample was distilled into one number (the mean); same with the standard deviation. The mean for each channel was displayed on the recording program GUI until the next sample was taken, where it was replaced with the more current measurements. Now the six pieces of information (3 means, 3 standard deviations) were concatenated into a new array; the time when the sampling began was also concatenated into the array.

The data flow then arrived at an if-then statement. If a new file was to be created, the data would be concatenated with an array that assigns labels to columns (Drag Mean, Drag Standard Deviation, Lift Mean, etc.). The data would then be written to a text file and saved. If the "append" option was selected, 
then the appropriate file would be opened, the data would be concatenated with it, and the file would be closed.

Meanwhile, the streaming program was running alongside the recording program. This allowed the measured variables to be observed graphically. Real-time streaming data is valuable because it allows the user to monitor the the signals before, during, and after recording. Sometimes the data lapsed into a "noisier" state during testing, rendering the entire test useless unless the noise level was brought down prior to writing the data to a file. The cause of this noise seemed to be related to the power supply of the display box. Any sudden change in the electrical power supply caused this noise. For example, turning any of the following devices on or off caused the data to become excessively noisy: strobotac, motor power supply and room lighting. When the circuit in the display unit was "tripped" into a state of excessive noise, the unit could simply be restarted using the power switch on the rear of the unit. It immediately reset and causes no problems.

The main advantage of having a LabVIEW program that manipulates data instead of just having it write to a spreadsheet, was the amount of time saved. Previous wind tunnel experimentation at Cal Poly has taken over eight hours to sift through in a normal test without the use of some sort of software like LabVIEW [21]. Once the data file was written, however, it may be opened using Microsoft Excel to be analyzed, manipulated, and graphed.

Prior to testing, a baseline was obtained by measuring the lift and drag without the golf ball in place. Then, the ball was placed in the wind tunnel and tested. The difference between these two values was taken to be the actual lift and drag forces. This is similar to what was done by Watts and Ferrer [34]. The following list of good testing procedures was derived from Pope (1954). 
1. Check all calibration curves before, during, and after each test.

2. Include a wide scope so there is no need to extrapolate data.

3. Find out how others do similar tests.

4. Be clear on data presented.

5. Have an acceptable level of calibrated zero-drift before beginning the test.

When possible, this list was used to guide the experimentation procedure. Pope (1954) is generally considered a definitive guide to low-speed wind tunnel testing which has been used for over 50 years. Other considerations that are mentioned are calculations for buoyancy, free stream turbulence, and blockage. The buoyancy and blockage of a golf ball were assumed to be negligible for this study; free stream turbulence may very well be important, but was not tested. See Section 5.8 for more information. 


\section{Chapter 4}

\section{Results}

Ideally, the flight coefficients would be obtained as functions of $S p$ and $R e$. These results could then be used to illuminate the problem with the existing data. Namely, why the flight coefficient curves seem to bend back on themselves. The apparatus was designed with the intent of providing accurate information in this regard.

\subsection{Lift and Drag Results}

The values of lift for Reynolds numbers ranging from 10,000 - 140, 000, corresponding to speeds of about $27-160 \mathrm{ft} / \mathrm{s},(8.2-48.8 \mathrm{~m} / \mathrm{s})$ were measured and are presented in Figure 4.1 and Figure 4.2. This graph was produced by measuring the lift for the system without the golf ball in place, then measuring lift with the ball in place, then taking the difference of the two sets of data. Sixty data points were taken at a $1.0 \mathrm{~Hz}$ interval. The data points for $R e<10,000$ were not included in the graph since the uncertainty for low Reynolds numbers becomes too high. Theoretically, the lift for the entire range of Reynolds numbers should 
be zero. The lift force due to the ball maintains a fairly constant value of $-0.2 l b_{f}$ $(-0.9 \mathrm{~N})$ until the the wind tunnel speed reaches $120 \mathrm{ft} / \mathrm{s}(36.6 \mathrm{~m} / \mathrm{s})$ at which point the force increases and changes sign. These results were not repeatable to within an acceptable margin; performing identical tests yielded results differing by up to $25 \%$.

In an effort to understand why this curve has this particular shape, the test was repeated under exactly the same conditions as well as with some minor changes (i.e. differently shaped fairing, allowing time for any "creep" effects to occur, etc.). The results were not in general repeatable, however, indicating a deeper problem. The possible problems, and some suggestions and solutions are outlined in Section 4.4 and Chapter 5. As a special note, no effects of creep were found. To test for this, a clock time was added to the DAQ program so that the relative time for each measurement was displayed in the output file. Several readings were taken under varying circumstances to test for any signs of creep, but none were found.

Once the force data was obtained, it was modified to produce dimensionless numbers (i.e. $C_{L}, C_{D}, R e, S p$ ). The following explicit equations were used to calculate the dimensionless parameters values'.

$$
\begin{aligned}
C_{L} & =\frac{F_{L}}{\frac{1}{2} \rho V^{2} A} \\
C_{D} & =\frac{F_{D}}{\frac{1}{2} \rho V^{2} A}
\end{aligned}
$$

The lift and drag coefficients were then plotted against the Reynolds number. Although the equipment was not able to produce useful results for measuring lift, the drag measurements are more informative. Compared with known information 


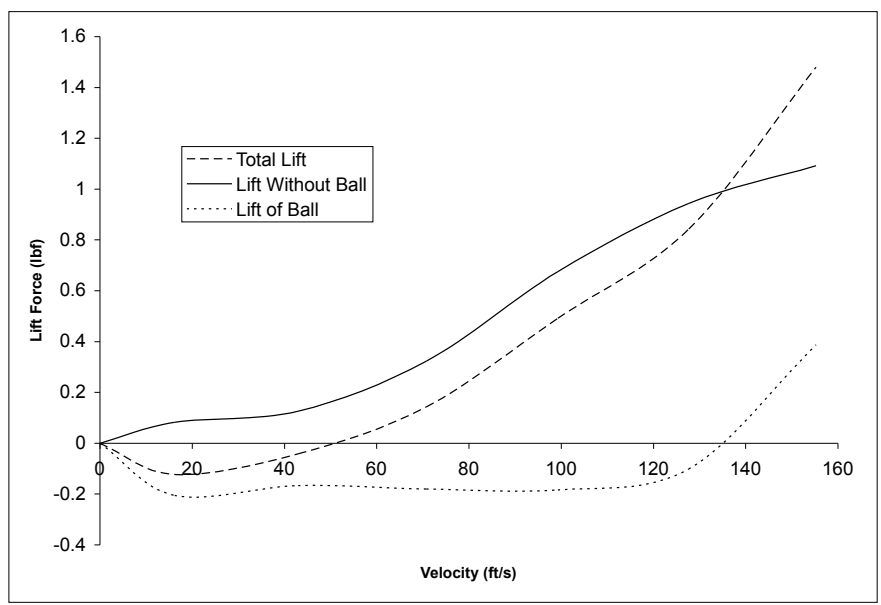

Figure 4.1: Graph of the lift force vs. wind tunnel speed for zero spin.

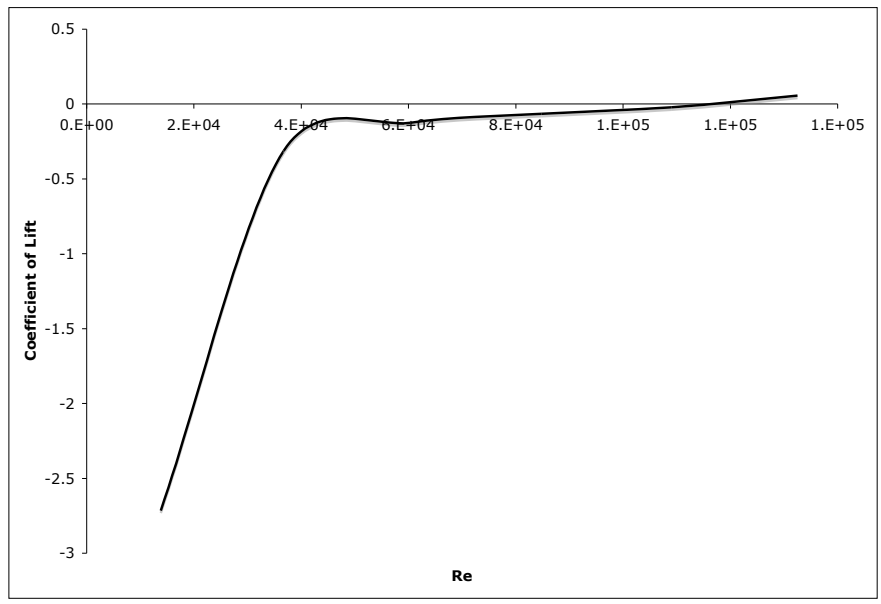

Figure 4.2: Graph of the coefficient of lift vs. Reynolds number for zero spin. 


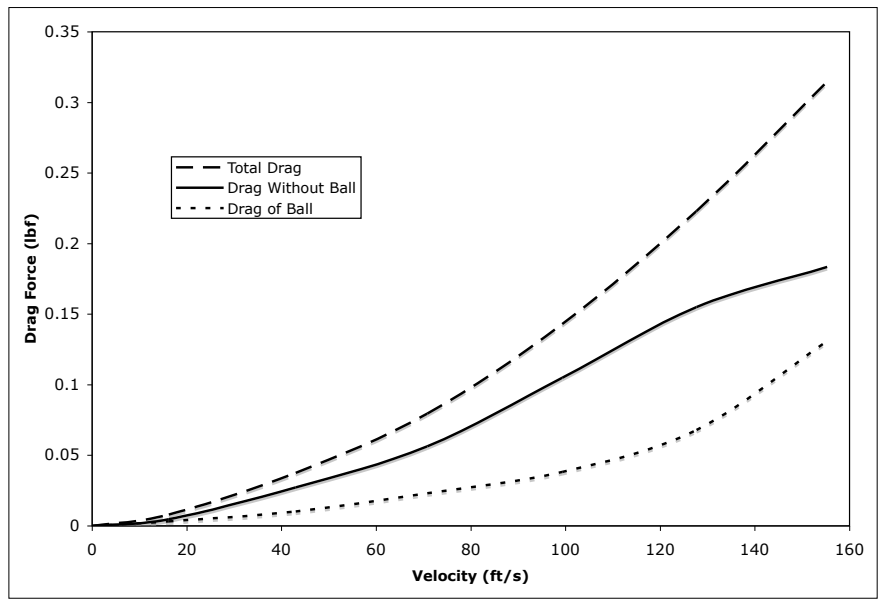

Figure 4.3: Graph of the drag force vs. wind tunnel velocity for zero spin.

on how a golf ball drag profile should look [13], the experimental profile is very similar in structure. As can be seen in Figure 4.4, the value for turbulent flow $(R e>40,000)$ is approximately 0.015 . Most other sources site a value of $C_{D}=$ 0.2. Therefore, it is assumed that the value presented here is erroneous. The measured value must suffer from the poor performance of the test equipment and should not be taken as accurate data. Unlike the lift data, the drag data is repeatable. The values have been consistently one order of magnitude lower than the expected value. More information on to why this is has been presented in Section 4.4 and Chapter 5 .

The proceeding data has been for a golf ball with no spin. When the same tests are done for a spinning ball, similar results are obtained. In full, the lift and drag forces for ten spin speeds $(0,1000,2000,3000,4100,5000,6000,7000,8000$, and $8600 \mathrm{rpm})$ and 60 free-stream velocities, $0-160 \mathrm{ft} / \mathrm{s}(0-48.8 \mathrm{~m} / \mathrm{s})$, at $2.7 \mathrm{ft} / \mathrm{s}$ 


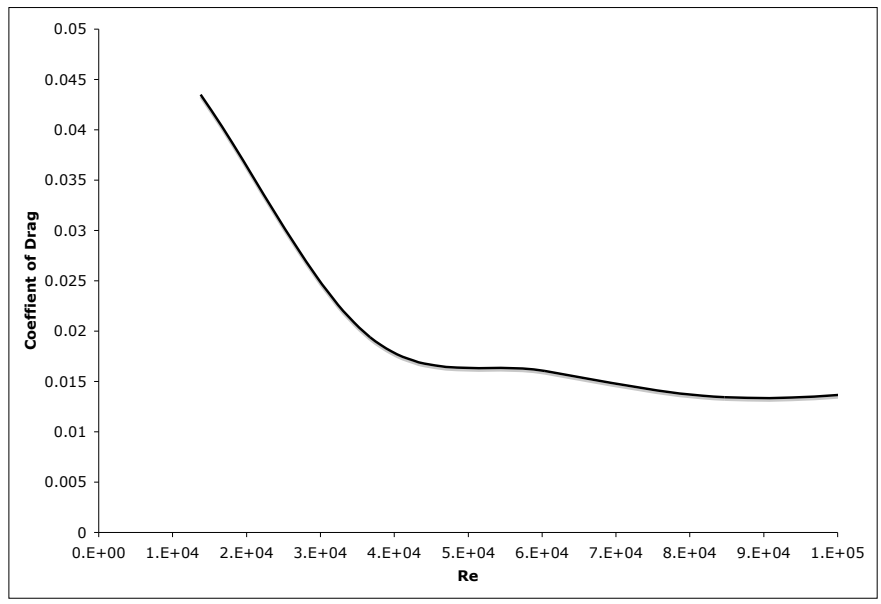

Figure 4.4: Graph of the coefficient of drag vs. Reynolds number for zero spin.

$(0.82 \mathrm{~m} / \mathrm{s})$ intervals were recorded for analysis. Notice that the testing was not done for 4,100 rpm. This is because there is a strong natural frequency at this speed. The shaft begins to visibly bend, and if the system is allowed to rotate at this speed for too long, there may be a very real possibility of failure of the shaft. At 4,100 rpm, however, the vibration dies down considerably, and it is safe to test at this speed. The intended result was that separate maps of $C_{D}$ and $C_{L}$ would be created using both $R e$ and $S p$ as abscissas. In light of the conclusion that only faulty values for lift and drag have been read, there is no benefit to presenting the data here.

\section{$4.2 \quad$ Vortex Shedding}

In the range of Reynolds numbers from 60 to 5,000 a body will cause vortices to shed from the flow $[11,10]$. This is due to the presence of the Kármán vortex 
street. The Strouhal number is a dimensionless parameter that characterizes the frequency of the vortex shedding, and it is a function of the Reynolds number for many bluff bodies [13]. The Strouhal number is defined by the following equation.

$$
S t=\frac{n D}{U}
$$

where $n$ is the frequency, $D$ is the diameter, and $U$ is the free-stream velocity. The Strouhal number takes on a constant value of $S t=0.21$ for $R e>10^{3}$ in the case of a circular cylinder. For the axle which spins the golf ball, the shedding frequency at $160 \mathrm{ft} / \mathrm{s}(48.8 \mathrm{~m} / \mathrm{s})$ is about $2,150 \mathrm{~Hz}$. This is a significant contributing factor to the noise of the measurement.

\subsection{Circulation}

One possible contributing factor to the change in flight coefficients is circulation. After the ball is hit, the velocity is initially much faster than the surface of the ball due to spinning. The ball's velocity decreases faster than its spin rate, however, and the circulation of air around the ball starts to contribute more and more to the overall action of the ball. If the ball spins fast enough, the flow becomes fully circulatory [4]. This critical condition is reached when $\kappa / a U=4 \pi$,

where $\kappa$ is the dipole strength, $a$ is the radius, and $U$ is the free-stream velocity. This condition is not met for the golf ball; the relevant data is presented in Figure 4.5 .

The dipole strength, $\kappa$, is not explicitly known for a golf ball. The expression for a smooth sphere is complex and may differ from that of a golf ball by a large margin [13]. Therefore, no attempt was made to calculate the critical value, but as can be seen from Figure 4.5, the tangential velocity of the golf ball's surface 


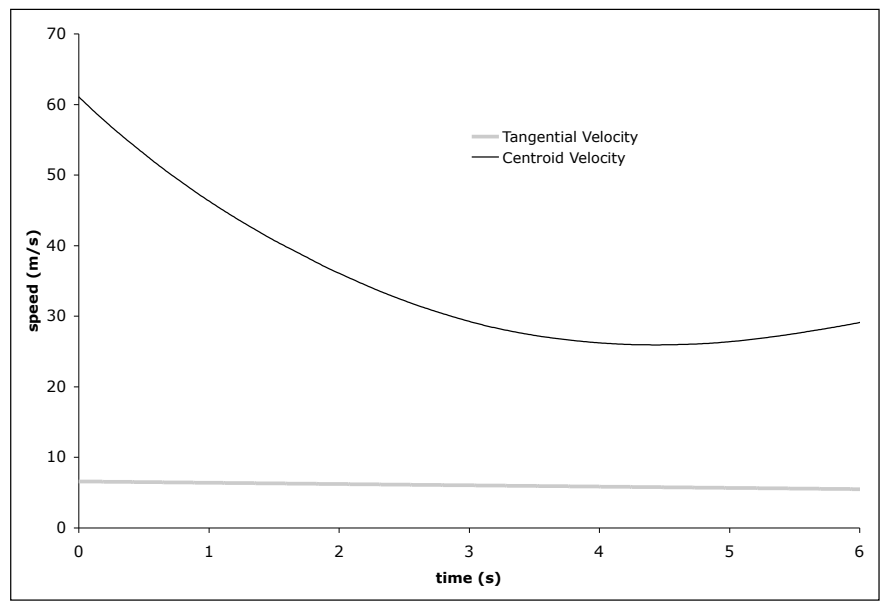

Figure 4.5: Graph of the velocity of the ball vs. the highest tangential velocity of the surface of the ball.

never even approaches the velocity of the free-stream flow. Still, this suggests that the circulation of the boundary layer may be a good place to start looking for the sudden transition of the lift and drag coefficients.

\subsection{Areas For Improvement}

While testing, a number of problems were encountered. Some were solved, others proved to be more complex. The root cause of all the problems seemed to be that the equipment being used for experimentation has limitations. The wind tunnel and its force transducers don't seem to allow for the precise nature of the experiments needed to measure lift and drag on a spinning golf ball. The two major reasons for failure to obtain precise data are highlighted here. 


\subsubsection{Fairing Gives False Readings}

Unexpected lift results were recorded after the initial testing. They were two orders of magnitude higher than what was expected. To shed some light on the problem, the sting was isolated and the tests were repeated. After completely enclosing the sting with the combination of the plastic fairing and a cardboard, airtight fairing, it became evident that the fairing itself was transmitting a measurable force.

The following test was conducted to investigate the problem. The entire force transducing fixture was secured into the calibration base. Instead of hanging weights from the sting, like one normally would, the weights were hung from the fairing itself. The results are shown in Figure 4.6 and Figure 4.7. When the weights were hung in the same direction the drag force would act, a force was measured by the transducer which would normally measure the lift of the sting. The measured force closely fir the equation $y=0.05 x^{2}$. Where $y$ is the measured number, and $x$ is the weight hung from the fairing.

The same test was also done to see if a "lift" force on the fairing would register any forces in the force transducer. This time a linear result was obtained. The equation $y_{0}=0.0067 x_{0}$ was found to approximate this relationship. Neither of the tests showed any correspondence between a force applied to the fairing and a reading on the transducer set to measure drag. In other words, any force applied to the fairing will read a false lift force, but will not register a false drag force.

These forces are on the same order of magnitude as the weight of the golf ball itself, making them impossible to ignore. Even though the forces have been characterized, it is very difficult to look at the measured data and account for this fairing interference by, say, subtracting these forces off from the total measured 


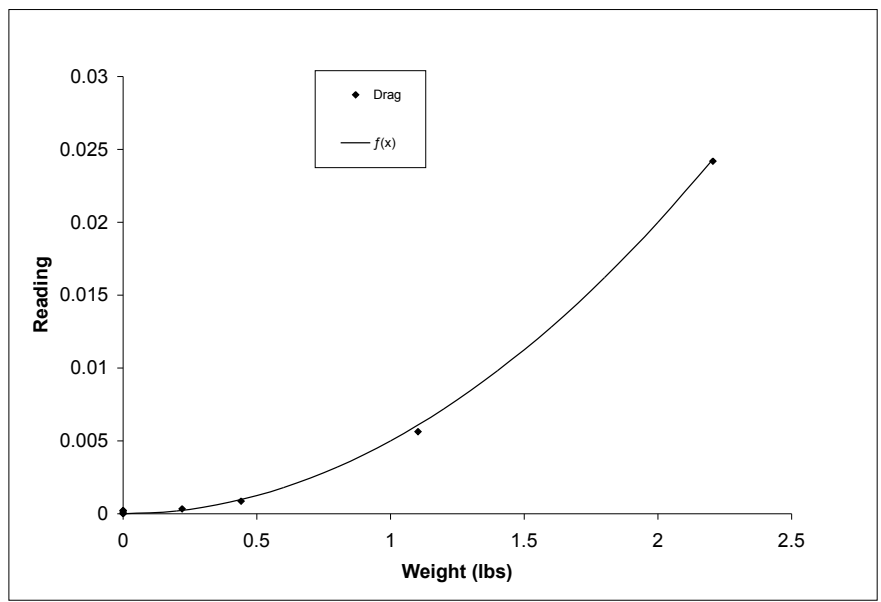

\section{Figure 4.6: Drag readings transmitted through the fairing.}

force. The reason is that even though these graphs clearly show that there is a relationship between a force reading and a fairing force, the fairing force cannot be correlated to a wind speed because the drag coefficient behavior for the fairing isn't known. It does not seem to be amenable to testing with this setup either, because the fairing is needed in place for accurate readings.

The source of this interference seems to be that the fairing is screwed directly into the base plate of the force transducing fixture. Within the fixture, there are two LVDTs. The LVDTs work by measuring the small displacement of a magnetic core relative to the coils that surround it. The LVDT that measures drag is "nestled" inside the fixture. but the LVDT that measures lift is directly attached to the fixture base, which in turn is directly attached to the wind tunnel test section. A force on the fairing causes bending in the fixture plate which in turn causes a displacement in the LVDT. 


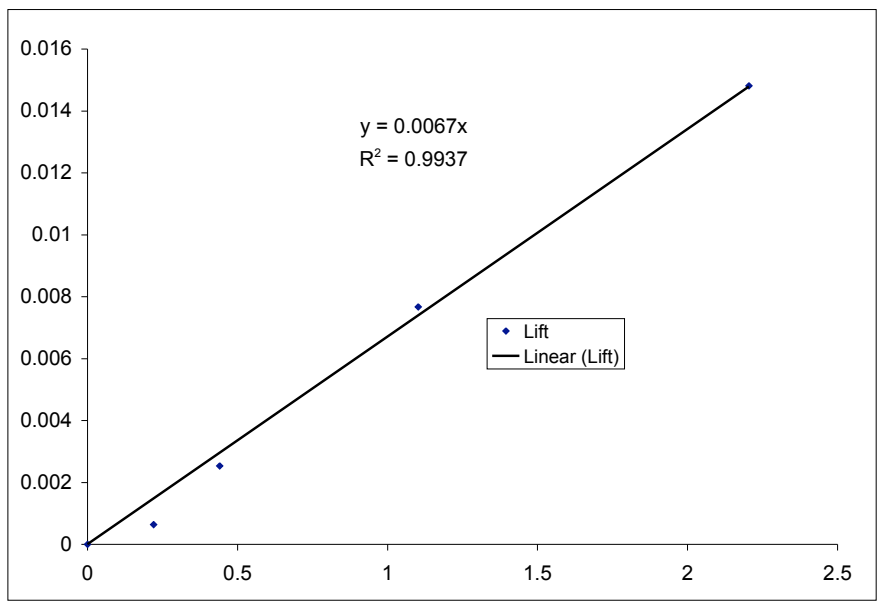

Figure 4.7: Lift readings transmitted through the fairing.

\subsubsection{Noise From the Fan Motor}

As mentioned in the above section, the magnetic core of the LVDT is screwed in to the fixture base, which is rigidly attached at the bottom of the wind tunnel test section. As the fan motor speed is increased, larger amplitude vibrations are transmitted through the entire structure of the wind tunnel. In particular, vibration in the test section starts to reach alarming levels above $30 \mathrm{~Hz}$. At low wind speeds, there is still some noise, but the amplitude of this signal is very small $(<0.01 \mathrm{~V})$. In steady-state, the signal read by the force transducers looks periodic with an amplitude much greater than the mean value of the signal. For example, while measuring a lift force, if $0.000 \mathrm{~V}$ (zero volts) is read at a wind speed of $0 \mathrm{ft} / \mathrm{s}$, a typical signal value at $100 \mathrm{ft} / \mathrm{s}(30.5 \mathrm{~m} / \mathrm{s})$ might be $0.200 \mathrm{~V}$. The amplitude of the signal, however, will be around $0.5 \mathrm{~V}$. So, at low speed a signal may have a mean of $0.010 \mathrm{~V}$ and an amplitude (due to unwanted noise) of $0.005 \mathrm{~V}$. At high speeds, the signal mean may be $0.150 \mathrm{~V}$, but an amplitude 
(still due to noise) may increase to $0.5 \mathrm{~V}$. The result is a very noisy signal.

Figure 4.8 shows an example of a typical signal recorded by the DAQ at $30 \mathrm{~Hz}$. The signal mean has moved from $0 \mathrm{~V}$ to about $-0.1 \mathrm{~V}$. But the maximum amplitude is about $0.4 \mathrm{~V}$. Surprisingly, some consistent results are still obtainable because the noise is due primarily to rotating machinery, which produces sinusoidal signals. Averaging at fast rates over long intervals tends to give mean values that at least appear to be correct. Any fine measurements, unfortunately, seem hopeless. To measure the lift and drag of a golf ball, as it turns out, fine measurements are necessary.

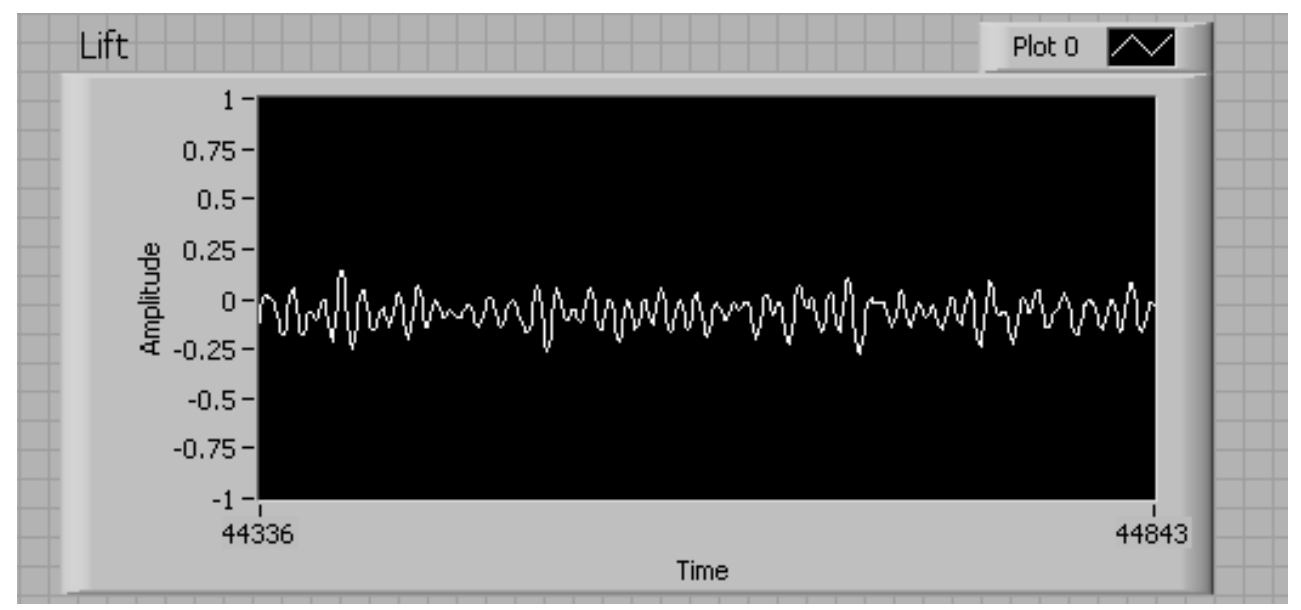

Figure 4.8: A typical signal showing a maximum-amplitude to mean ratio of greater than unity.

\subsubsection{Solutions}

To these problems, the following solutions might be suggested. A re-design of how the fairing attaches to the base. Currently, the fairing uses four screws to rigidly attach to the base of the transducer fixture. Some softer cushions (cardboard, felt, air gap) were inserted in between the two parts and tested, but 
this did not help. Ideally, the fairing needs to not be attached to the fixture at all. One solution would be to attach it directly to the test section's bottom wall. This requires drilling holes directly into the wind tunnel. As an alternative to drilling holes, the wind tunnel could be adhered to the wind tunnel. This brings up more questions, however, to the stability of such a design, damage to the wind tunnel, and more disturbance to the flow. For the current golf ball setup, this would raise the height of the fairing and would cause mechanical interference with the bearing housing.

As for the noise problem, generally isolating the fixture from the wind tunnel should work well. One solution would be to use a tripod to secure the fixture. It would have to be modified to securely hold the fixture. This would be expensive and would no doubt give rise to more problems. For example, now there would be an air gap in between the fixture and the test section. This would need to be blocked by something that would not transmit any vibration to the very sensitive LVDTs. Also, the tripod must be so rigid as to have negligible deformation (or rigid body displacement) so that all of the sting's motion is captured by the LVDTs.

There are many additional problems that have been outlined in the thesis (see Chapter 5), but they have either been solved, accounted for, or are so small that they do not pose a threat to the validity of the results. There is still hope of measuring the desired properties, but more time and funding is needed. The author estimated that another year of work would yield good results if the research were to be continued. 


\section{Chapter 5}

\section{Suggestions}

This chapter discusses the problems encountered with the use of the wind tunnel for measuring golf ball lift and drag forces. It should serve as a troubleshooting guide for anyone who is using the tunnel. The problems described herein have either been solved or a solution has been recommended but not implemented due to time or funding constraints. The logical continuation of this project would consist of solving many of the problems mentioned here.

\subsection{Pitot Tube Mount}

There is a Pitot tube mounted vertically onto the upper wall of the test section. The tube protrudes into the tunnel through a slit. A 2-axis guide rail system allows manual movement of the Pitot tube in the z-direction and the x-direction. Plastic tubes transmit the pressure from the Pitot tube to the transducer in the control box. During operation, the tube's support structure starts to vibrate which may affect the noise of the LVDTs and also the Pitot tube's pressure reading. There are plans to automate the Pitot tube's movement, 
and it is suggested that the support needs to be made stiffer.

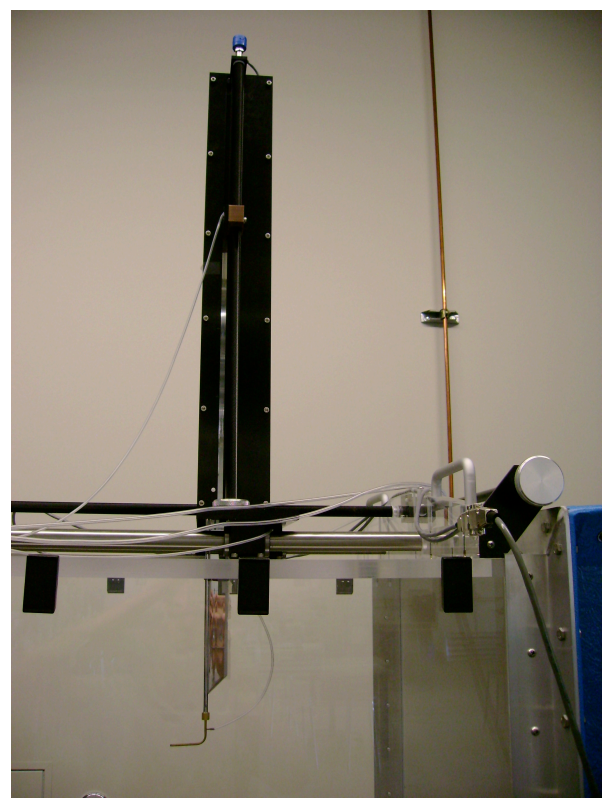

Figure 5.1: Photograph of the Pitot tube mount and Pitot tube extending into the wind tunnel.

\subsection{Pitot Tube Slit}

As mentioned in the previous paragraph, the Pitot tube enters the wind tunnel via a slit on the test section's upper surface. This slit measures approximately $1.25 \mathrm{~cm} \times 100 \mathrm{~cm}$. Recalling that the pressure inside the running wind tunnel is significantly lower than the ambient, air tends to be sucked into the test section. In an attempt to negate this flow, the slit is lined with bristles which block some of the flow while still allowing the Pitot tube to operate normally. Unfortunately, a large amount of fast moving air flows past the bristles and into the tunnel during normal operation. In order to block the air from entering the wind tunnel, a flat plastic piece of material was set over the slit. Figure 5.2 shows what happens to the measurements when the slit is blocked. In the figure, a typical lift curve is 
shown for the golf ball with and without blocking the silt. At low speed there is not much difference, but at speeds of around $115 \mathrm{ft} / \mathrm{s}(35 \mathrm{~m} / \mathrm{s})$ the difference in lift differs by $33 \%$.

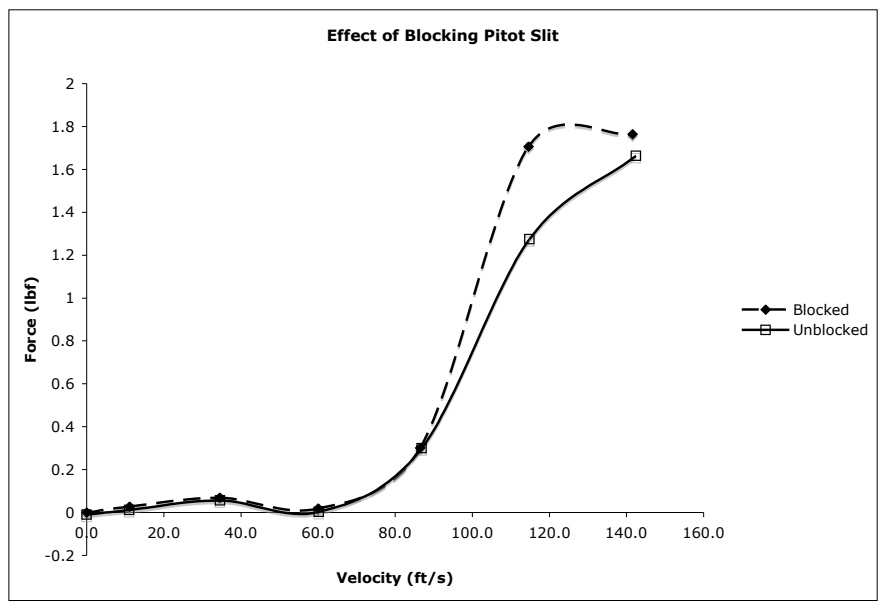

Figure 5.2: Graph of Lift vs. Velocity for an arbitrary object with the Pitot slit (a) unblocked, and (b) with the slit blocked so as to allow no air to enter through the top of the tunnel.

\subsection{Bernoulli Effect}

One interesting source of lift is a particular Bernoulli effect. When there is nothing on the sting, the top of the sting acts like a flat plate. The bottom of the sting is exposed to ambient conditions, while the top is exposed to the conditions inside the wind tunnel. Since air is passing over the sting with a high velocity, it has a lower-than-ambient pressure described by the following equation:

$$
\Delta p=\frac{1}{2} \rho V^{2}
$$


where $\Delta p$ is the pressure difference between the top and bottom surfaces of the sting, $\rho$ is the density of air and $V$ is the free stream velocity in the tunnel. We also know that the bernoulli force on the sting, $F_{b}$, is the pressure difference times the area, $A$, of the sting's top surface. Combining $F=\Delta p A$ with eq. 5.1, we obtain

$$
F_{b}=\frac{1}{2} \rho V^{2} A
$$

The Bernoulli force [8] is proportional to velocity squared, that is, $F_{b} \propto V^{2}$. Figure 5.3 compares the curve from Equation 5.2 alongside the measured result. The two curves are in close agreement, suggesting that the Bernoulli force is a major contributor to the total force on the sting. Still, the curves diverge at high speeds which implies that there are other effects not yet taken into account. The other important effects are the fairing interference and suction effects discussed below.

\section{$5.4 \quad$ Fairing}

The purpose of the fairing is to block the freestream air from contacting the bare sting and producing a force reading. As shown through testing, however, any force on the fairing does cause the lift LVDT to read a lift force. When no model is attached to the sting, the force transducer reads a negligible amount of drag, but a large amount of lift. There seems to be no way to compensate for this. Several different fairings were made to attach to the main fairing of the wind tunnel. The one pictured in Figure 5.4 is a small fairing that protects the bearing mount from being directly in the flow field. The intended effect was to 


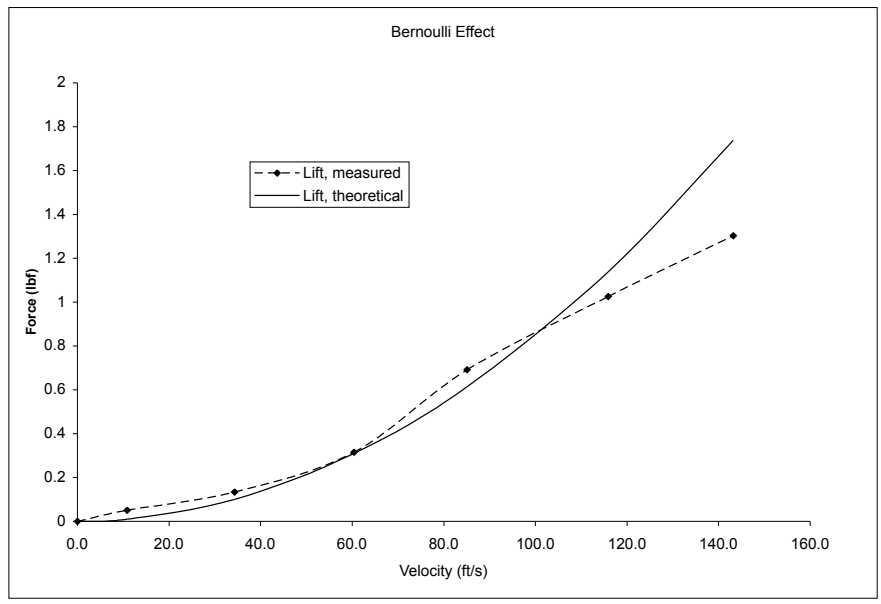

Figure 5.3: Graph of Lift vs. Velocity for the bare sting. The theoretical Bernoulli effect is shown alongside the measured data

cut down the drag of the apparatus to get a better baseline for testing. Although the additional fairing greatly reduced the drag, the lift actually become greater in magnitude. This is because the fairing itself registers a lift force (see Section 4.4.1. Another fairing that was used is shown in Figure 5.7. There seems to be no easy solution to the problem of fairing interference, but some solutions were presented in Section 4.4.3. This is the largest obstacle in the way of producing good results from the test equipment.

\subsection{Mechanical Interference}

The lift sensor will sometimes measure a negative lift for small Reynolds numbers $(<40,000)$ when it should register a positive lift. The reason is due to some backlash in the adjustment screws. There are screws that, on one end are attached to the LVDT cores, and on the other are screwed into the immobile 


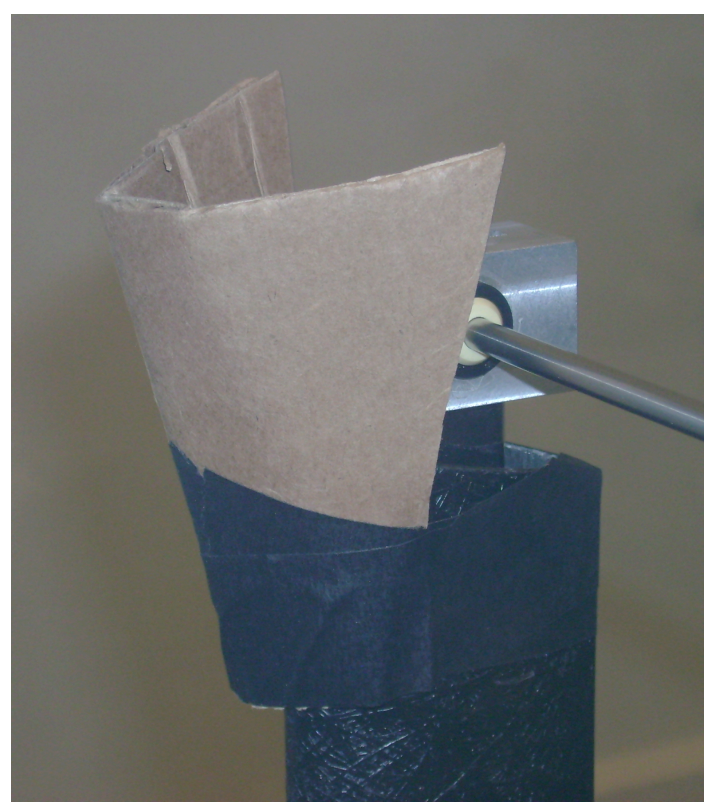

Figure 5.4: Photograph of a simple fairing made from cardboard to minimize disruption of the flow field.

platforms. There is also a plastic tightening screw for each LVDT so that the adjustment screw is not easily turned. This ensures a minimum amount of "zerodrift" during testing. The tightening screws are not perfect, however, and the adjustment screws have some wobble and backlash. By wobble, it is meant that while turning the adjustment screw, the core wobbles so as to not stay perfectly concentric with the outer coils. It is not known how this effects the reading.

\subsection{Suction Through Fairing}

During normal operation, air is drawn along the sting and inside the fairing from the ambient to the test section. The wall shear stress on the sting contributes to an upward lift force. As the air flows out of the top of the fairing it no doubt contacts whatever body is attached to the sting. In this case, it is the bearing mount that is bolted onto the sting. There must be some drag due to this flow, 


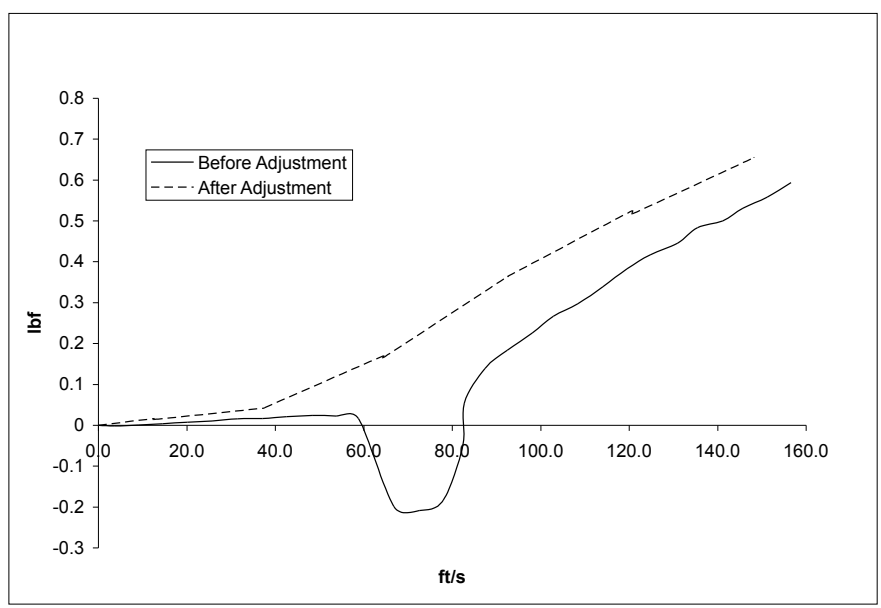

Figure 5.5: Mechanical Interference Comparison. The solid line was the recorded information originally. The dashed line is the recorded curve after the sting apparatus had been taken apart with tightened adjustment screws, shortened calibration screws, and re-positioned LVDTs 
and it is in general not easy to say how this sub-flow will effect the results of the test. A strip of latex (made from a standard balloon) was used as a barrier to keep out air from entering the fairing. While this system blocked most if not all the leaking air into the wind tunnel it is not clear what force was transmitted to the sting via the latex seal.

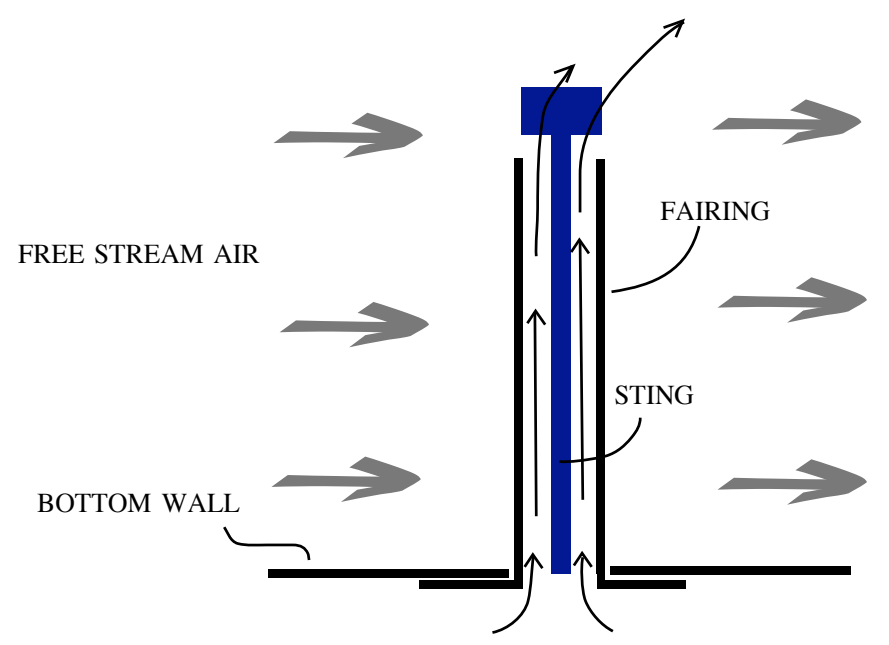

Figure 5.6: Illustration of the path that air takes as it is sucked through the fairing and into the test section. This causes an additional lift force that is difficult to measure.

In order to test the magnitude of this force, a fairing was constructed out of cardboard (see Figure 5.7) to completely enclose the exposed part of the sting, situated just above the fairing. A few small holes were created in the fairing so that the air could still pass through. Then, the holes were covered to make the fairing air-tight. These two lift and drag measurements were then compared to find the effect of the leak. It was found that the effect was rather small compared to the lift caused by the fairing; about an order of magnitude less. Still this effect is important to understand, especially if different fairings are to be used. 


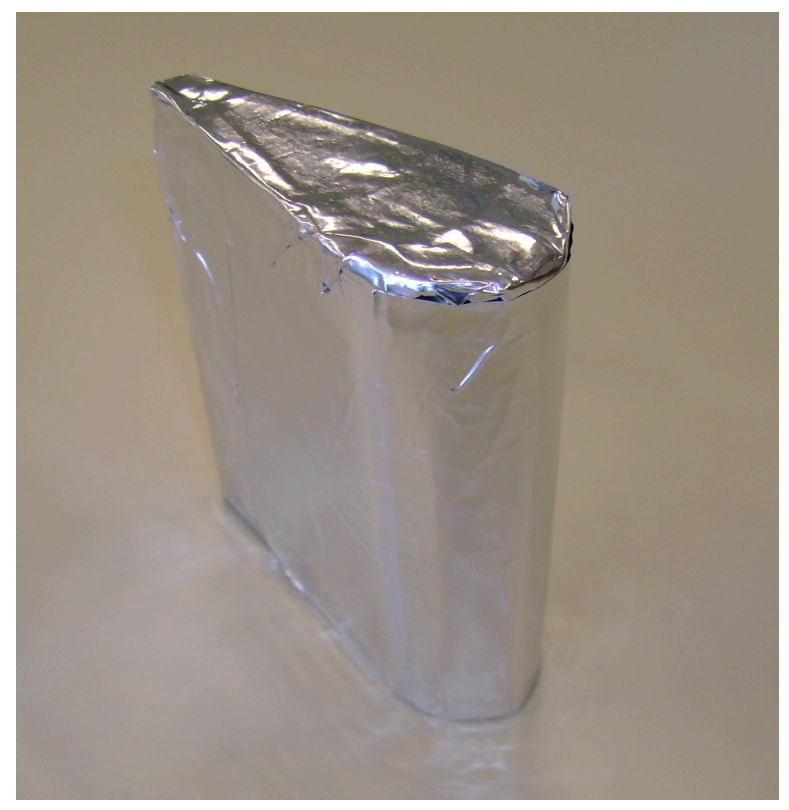

Figure 5.7: A photograph of a fairing made from cardboard with taped seems to prevent air from flowing into the wind tunnel through the sting body. Aluminum was used to give the body a smoother surface.

\subsection{Force Transducer Isolation}

The major source of noise of the wind tunnel was due to the vibration of the fan motor. Because of the geometry of the force transduction assembly, the lift LVDT was greatly affected by the fan vibration, and the drag LVDT was much more isolated. As shown in Figure 5.8, the standard deviation of the drag signal stayed fairly constant over the range of wind speeds. The lift signal, on the other hand, got very noisy as the the fan motor speed is increased. This can be seen as a steady increase in the standard deviation of the signal as the wind speed is increased. Currently, the whole force transduction unit is flush with the test section. Much of the vibration generated from the wind tunnel is being measured by this unit. If the system was instead mounted to, say, a tripod, the vibration produced by the wind tunnel would not be recorded. 


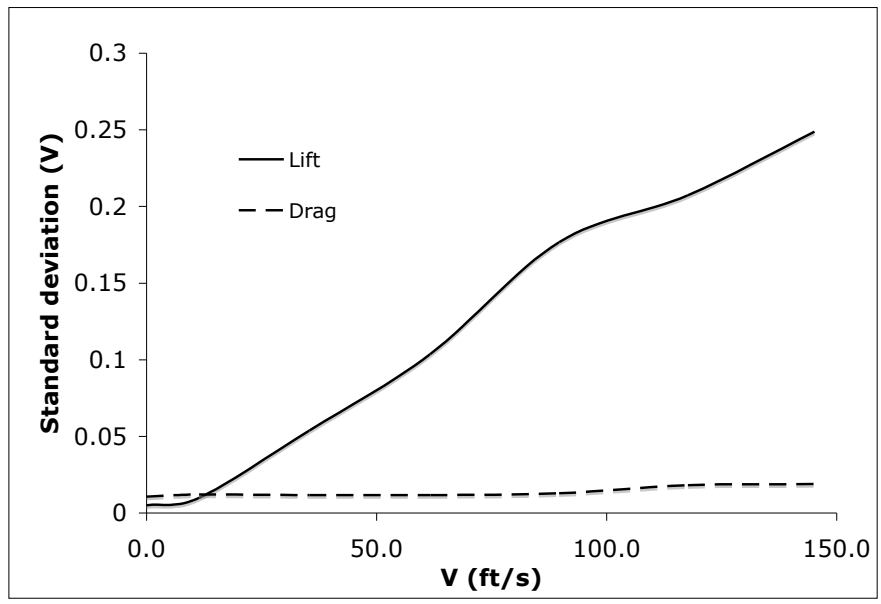

Figure 5.8: Standard Deviation Comparison. This graph plots the standard deviation of the signals on channels 0 and 1 (drag and lift, respectively).

\subsection{Hot Wire Anemometer}

A more accurate way to obtain velocity data would be to use hot wire anemometry. The Pitot tube being used cannot accurately characterize the turbulence in the wind tunnel; especially the free-stream turbulence. This type of measurement has become standard for much of the ongoing turbulence research [6]. This modern approach would bring many advantages.

1. The sensors are very small and less intrusive than a Pitot tube.

2. Measurements can be taken at up to $1 \mathrm{MHz}$.

3. All three components of velocity can be measured simultaneously.

4. Very good accuracy as long as proper calibration is done. 
5. Low noise amplitude. Usually less than $0.01 \%$ of the measured value.

6. Easy integration with the existing DAQ.

Additionally, this is the best method to check for the free-stream turbulence inherent to the wind tunnel's operation. The traditional method is to use a smooth sphere. The drag force on the sphere is measured for a range of Reynolds numbers. The Reynolds number at which the drag on the sphere is 0.3 is deemed the critical Reynolds number, $R e_{C}$ and is 385,000 in the ideal case of pure, laminar free-stream flow. If there is indeed some free-stream turbulence, then transition will occur at a lower Reynolds number. The turbulence factor is defined as $T F=385,000 / R e_{C}$. Turbulence factors range from about 1.0 to 3.0 with values over 1.4 indicating that the flow may contain too much turbulence for accurate results [25]. For the wind tunnel used in this study, a sphere with a diameter of at least $6 \mathrm{in}$. is needed for the correct Reynolds numbers. Unfortunately, the largest available sphere was only $3 \mathrm{in} .(7.6 \mathrm{~cm})$ in diameter. 


\section{Chapter 6}

\section{Conclusion}

In summary, the design-build aspects of this project were completed, but the research portion proved to be beyond the capabilities of the current wind tunnel. Mechanical hardware was designed using SolidWorks software, fabricated in the Cal Poly machine shop by the author and student employees of the shop, and assembled into the wind tunnel for the express purpose of measuring the aerodynamic properties of golf balls under laboratory conditions. Additionally, software was developed using LabVIEW to aid in data acquisition and analysis of results. The DAQ is capable of recording large files $(>20 M b)$ with a high sampling frequency $(>20,000 \mathrm{~Hz})$. Voltage was measured which corresponds to mechanical force and pressure.

The system can spin a golf ball at speeds up to 8,600 rpm while keeping vibration down to acceptable levels. During operation, the golf ball was clearly visible and the rotational speed may be measured using a strobing tachometer approach. The DAQ is robust and easy to use. There are safety features built in to the software so that the chance of accidently overwriting files is kept to a minimum. Besides logging data, the LabVIEW program displays real-time values 
for drag, lift, and pressure. Physically, the system was quick to set up and small enough to be taken apart and stored near the wind tunnel. All of the parts are robust enough so that taking down the system should not cause any damage to the functionality of the device.

All of the custom hardware, such as the motor plate, bearing mounts, supports, and axles met or exceeded expectations with regard to their functionality. The pre-existing hardware of the wind tunnel on the other hand caused some problems with the data acquisition process. The Mechanical Engineering wind tunnel is an adequate piece of equipment for use as a pedagogical tool, but it is not advanced enough for scientific research. It was found that forces produced by a golf ball in this situation are much smaller than the forces produced by the auxiliary equipment, making the accurate measurement of lift and drag a very difficult task. The results of the experiments left much to be desired. The ultimate goal would be to have the ability to calculate the lift and drag coefficients as functions of Reynolds and spin numbers to shed some light on this unsolved problem. At this point, however, several aspects of the system remain to be improved.

Several suggestions were mentioned with enough detail so that the project may be continued at a later time without much loss of continuity. Further experimentation could involve securing the Pitot tube mount, covering the Pitot tube slit, countering the sting's Bernoulli effect by creating a full fairing, completely replacing the way the fairing connects to the rest of the apparatus, a re-design of the force transducer housing to get rid of backlash, a non-invasive method for blocking air from traveling through the fairing into the wind tunnel, mounting the force transducer housing to an isolated structure, and finally, incorporating hot wire anemometers into the DAQ. 
Another future development may be flow visualization. Currently, some work is being done to outfit the wind tunnel with some means to visualize the flow field. This would prove very valuable to understanding golf ball aerodynamics, especially in lieu of good numerical data. This document has been written to serve as a manual for using the equipment with special emphasis on understanding what, exactly, is currently possible, and what may be possible with the listed improvements. 


\section{References}

[1] K. Aoki, Y. Nakayama, T. Hayasida, N. Yamaguti, and M. Sugiura. Flow characteristics of a golf ball using visualization techniques. In Science and Golf III: Proceedings of the World Scientific Congress of Golf, Champaign, Illinois, USA, 1998. Human Kinetics Publishers.

[2] T. Asai, K. Seo, O. Kobayashi, and R. Sakashita. Fundamental aerodynamics of the soccer ball. Sports Engineering, 2007.

[3] S. Barber, S. Haake, and M. Carré. Using CFD to understand the effects of seam geometry on soccer ball aerodynamics. American Journal of Physics, 2006.

[4] G. K. Batchelor. An Introduction to Fluid Dynamics. Cambridge University Press, New York, NY, USA, 1967.

[5] P. W. Bearman and J. K. Harvey. Golf ball aerodynamics. The Aeronautical Quarterly, 1976.

[6] N. P. Cheremisinoff and P. N. Cheremsisnoff. Flow Measurement for Engineers and Scientists. Marcel Dekker, Inc., New York, NY, USA, 1988.

[7] H. W. Coleman and S. E. W. Glenn Steele. Experimentation and Uncertainty 
Analysis for Engineers. John Wiley and Sons, Inc., New York, NY, USA, 1999.

[8] C. T. Crow, D. F. Elger, and J. A. Roberson. Engineering Fluid Mechanics. John Wiley and Sons, Inc., New York, NY, USA, 2001.

[9] J. M. Davies. Aerodynamics of golf balls. Journal of Applied Physics, 1949.

[10] B. Fornberg. Steady viscous flow past a cylinder and a sphere at high reynolds numbers. In Boundary-Layer Separation, Berlin, Heidelberg, Germany, 1986. Springer-Verlag.

[11] R. J. Goldstein, editor. Fluid Mechanics Measurements. Hemisphere Publishing Corporation, New York, NY, USA, 1983.

[12] G. L. Heinrich. Evaluation of the Magnus Effect. California Polytechnic University, San Luis Obispo, 1973. Senior Project.

[13] E. L. Houghton and P. W. Carpenter. Aerodynamics for Engineering Students, Fifth Edition. Butterworth-Heinemann, Oxford, England, 2003.

[14] J. C. R. Hunt, J. C. Vassilicos, and N. K. R. Kevlahan. Turbulence: A state of nature or a collection of phenomena? In Progress in Turbulence Research, Washington, DC, USA, 1994. American Institute of Aeronautics and Astronautics, Inc.

[15] E. L. D. Inc. Wind Tunnel Manual. Engineering Laboratory Design Inc., 2007.

[16] M. R. Malik. Stability theory for laminar flow control design. In Viscous Drag Reduction in Boundary Layers, Washington, DC, USA, 1990. American Institute of Aeronautics and Astronautics, Inc. 
[17] D. D. Marshall. Modern techniques in computational fluid dynamics. Associate Professor, Aerospace Engineering, ddmarsha@calpoly.edu.

[18] D. T. Mase. Private conversation. Associate Professor, Mechanical Engineering,gmase@calpoly.edu.

[19] T. Mase. Radar data. Associate Professor, Mechanical Engineering, gmase@calpoly.edu.

[20] R. D. Mehta. Aerodynamics of sports balls. Annual Review of Fluid Mechanics, 1985.

[21] R. Ogunyoku. Evaluation of the Magnus Effect. California Polytechnic University, San Luis Obispo, 1998. Senior Project.

[22] Onaraighl. Os_schematic.png. Public Domain. Obtained from wikipedia.org.

[23] S. Patton. Orr-sommerfeld linear stability equations. Notes from a graduate level fluids class (Viscous Flow) with Scott Patton, PhD.

[24] E. Pierce. Lvdt.png. GNU Free Documentation License. Obtained from wikipedia.org.

[25] A. Pope. Wind-Tunnel Testing. John Wiley and Sons, Inc., New York, NY, USA, 1947.

[26] S. J. Quintavalla. A generally applicable model for the aerodynamic behavior of golf balls. 2002 .

[27] U. S. G. A. Research and T. Center. The Indoor Test Range (ITR) Tedhnical Description and Operation Manual. United States Golf Association Research and Test Center, 2001. 
[28] G. S. Shairer. Some opportunities for progress in aircraft performance 27th wright brothers lecture. Journal of Aircraft, 1964.

[29] K. Shollenberger. Wind tunnel data. Dr. Shollenberger, Cal Poly San Luis Obispo, has found the vertical velocity profile within the wind tunnel. Also, she has correlated wind speed with motor drive frequency. Associate Professor, Mechanical Engineering, kshollen@calpoly.edu.

[30] G. C. Symons. An Investigation of Static Pressure Around a Rotating Cylinder (Magnus Effect). California Polytechnic University, San Luis Obispo, 1958. Senior Project.

[31] J. C. Tannehill, D. A. Anderson, and R. H. Pletcher. Computational Fluid Mechanics and Heat Transfer. Taylor and Francis, Ltd., Philadelphia, PA, USA, 1997.

[32] G. Tavares, K. Shannon, and T. Melvin. Golf ball spin decay model based on radar measurements. Science and Golf III: Proceedings of the 1998 World Scientific Congress of Golf, 1998.

[33] USGA. The rules of golf. For public use. Obtained from http://www.usga.org/equipment/guide/book/appendix3ball.html.

[34] R. G. Watts and R. Ferrer. Lateral force on a spinning sphere: Aerodynamics of a curveball. American Journal of Physics, 1987. 


\section{Index}

ball-drop method, 13

bearing, 30

bearings, 23

Bernoulli effect, 51

boundary layer, 4, 15, 18, 20, 43

buoyancy, 36

calibration, 32

CFD, 4

channel, 22

channels, 29

circulation, 42,43

coupling, 27

creep, 38

cyanoacrylate, 24

DAQ, 17, 28, 32, 60

Data Acquisition System, see DAQ

drag, $7,8,40,41$

fairing, 21, 22, 47, 53

force transducer, 18, 44

frequency, sampling, 34

golf ball flow around, 14, 40, 49

spinning of, 29, 42

GUI, 34

hot wire anemometer, 58

ITR, 10

Kármán vortex street, 42

LabVIEW, 28, 30, 35, 60

lift, 7, 41, 44

linear variable differential transformers, see LVDT

linearized perturbation equation, 14

LVDT, 21, 45, 46, 52, 54

Magnus effect, 11

National Instruments, 28, 30, 33

natural frequency, 29

Navier-Stokes equations, 14, 15

Newton-Raphson, 10

Orr-Sommerfeld equation, 15

Pitot tube, 49 
Poiseuille flow, 15

pressure tap, 13

program

recording, 33

streaming, 35

radar, 8, 11

Reynolds number, 7, 14, 37, 41

uncertainty of, 37

separation, 13, 15

shaft, $25,27,30$

shear stress, wall, 54

SolidWorks, 60

sphere, 4, 8, 15

spin number, 4, 7, 37, 41

stability, 14

stagnation point, 13

standard deviation, 29

Strobotac, 30

Strouhal number, 42

test section, 46

trajectory, 7

turbulence, 4, 14, 15, 36, 59

transition, 13

USGA, 10

variable frequency drive, 18 vibration, 46

visualization, 62

wind tunnel, 17, 21, 27, 36, 61 


\section{Appendix A}

\section{Drawings}

\section{A.1 Final Concept}

These drawings are what was used to build the parts needed for experimentation. The actual parts deviate from the original drawings due to the iterative nature of this work. Examples include different size screws specified, tolerances, and even materials. These parts were made either by hand using a mill, or by a CNC mill. The stock acrylic material was purchased from McMaster-Carr. 


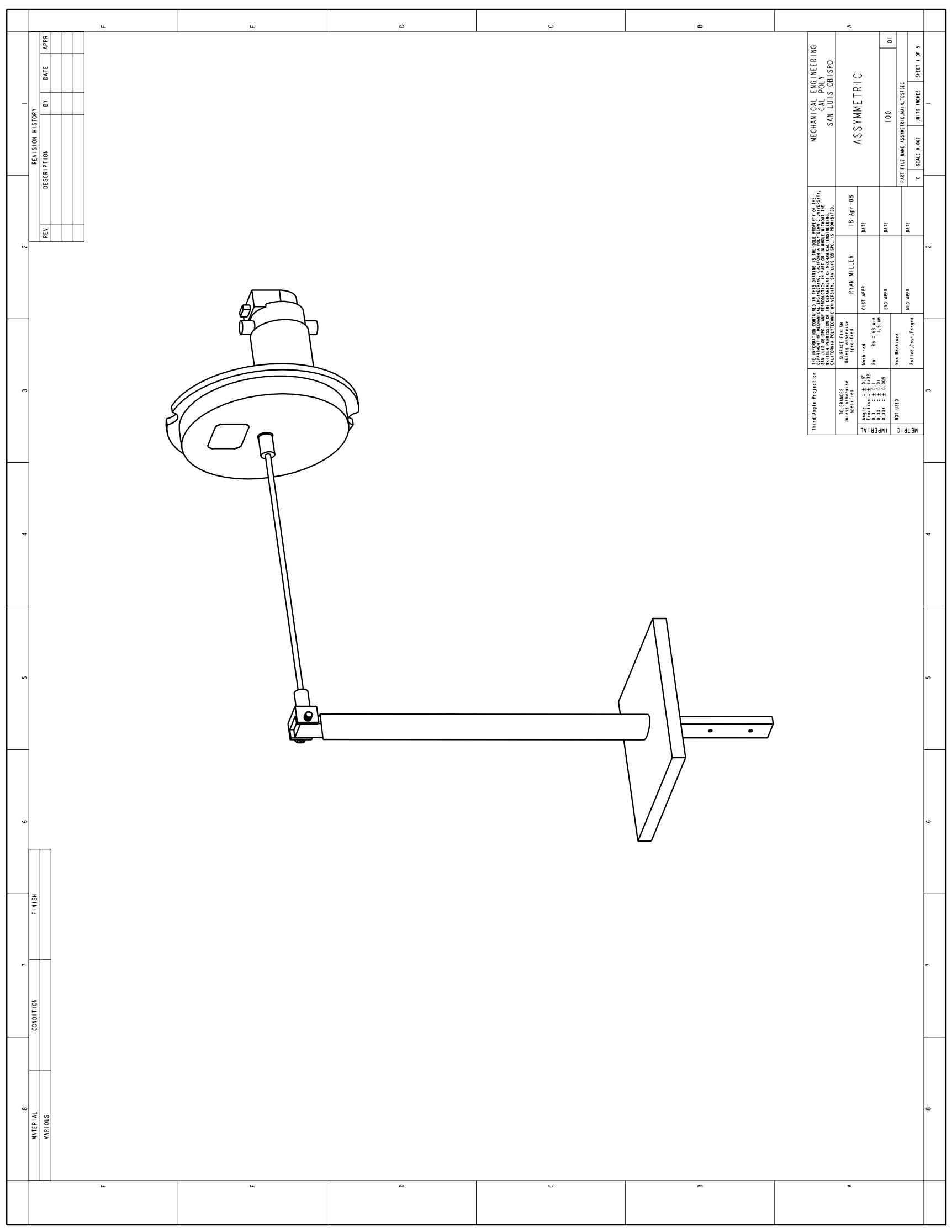




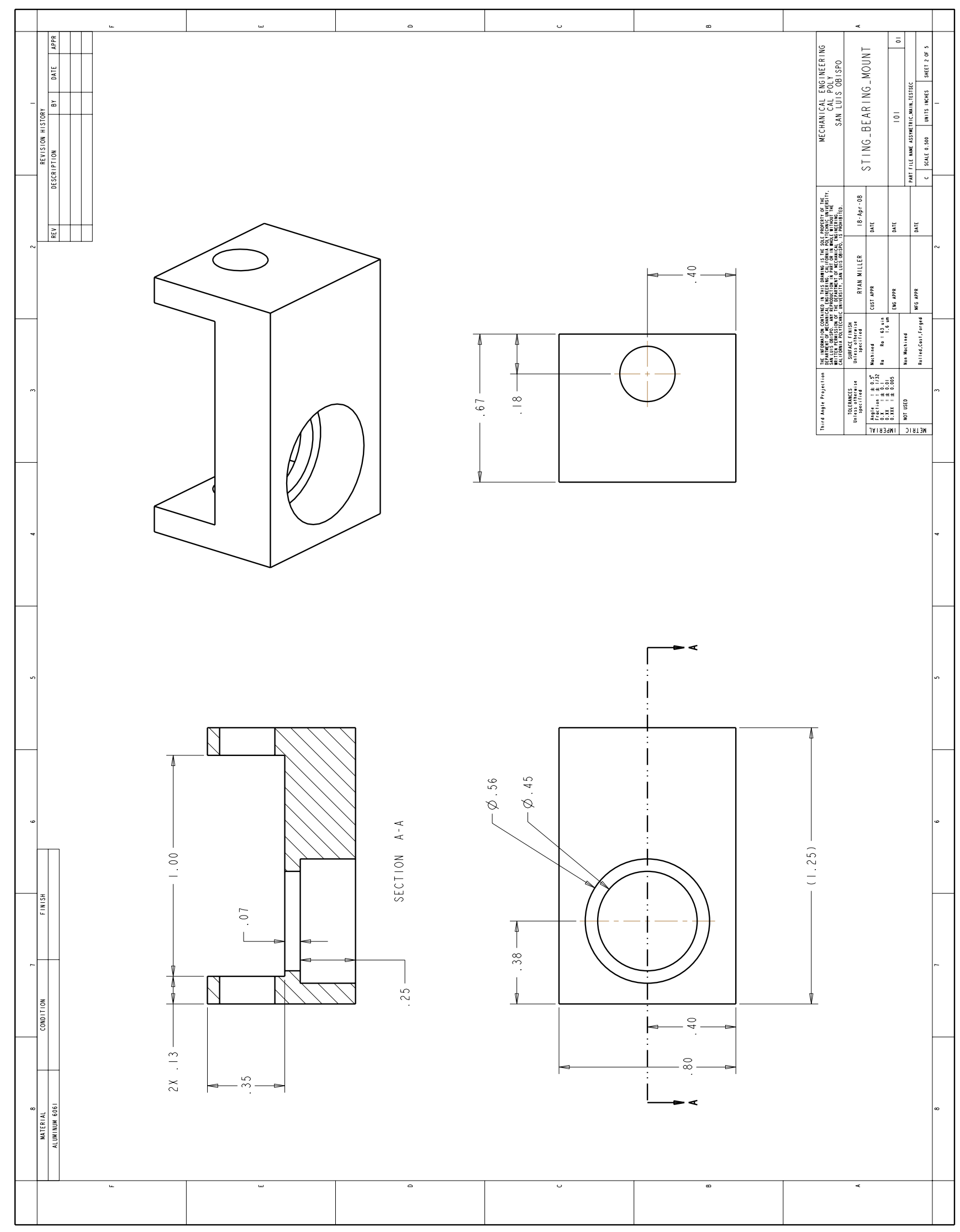




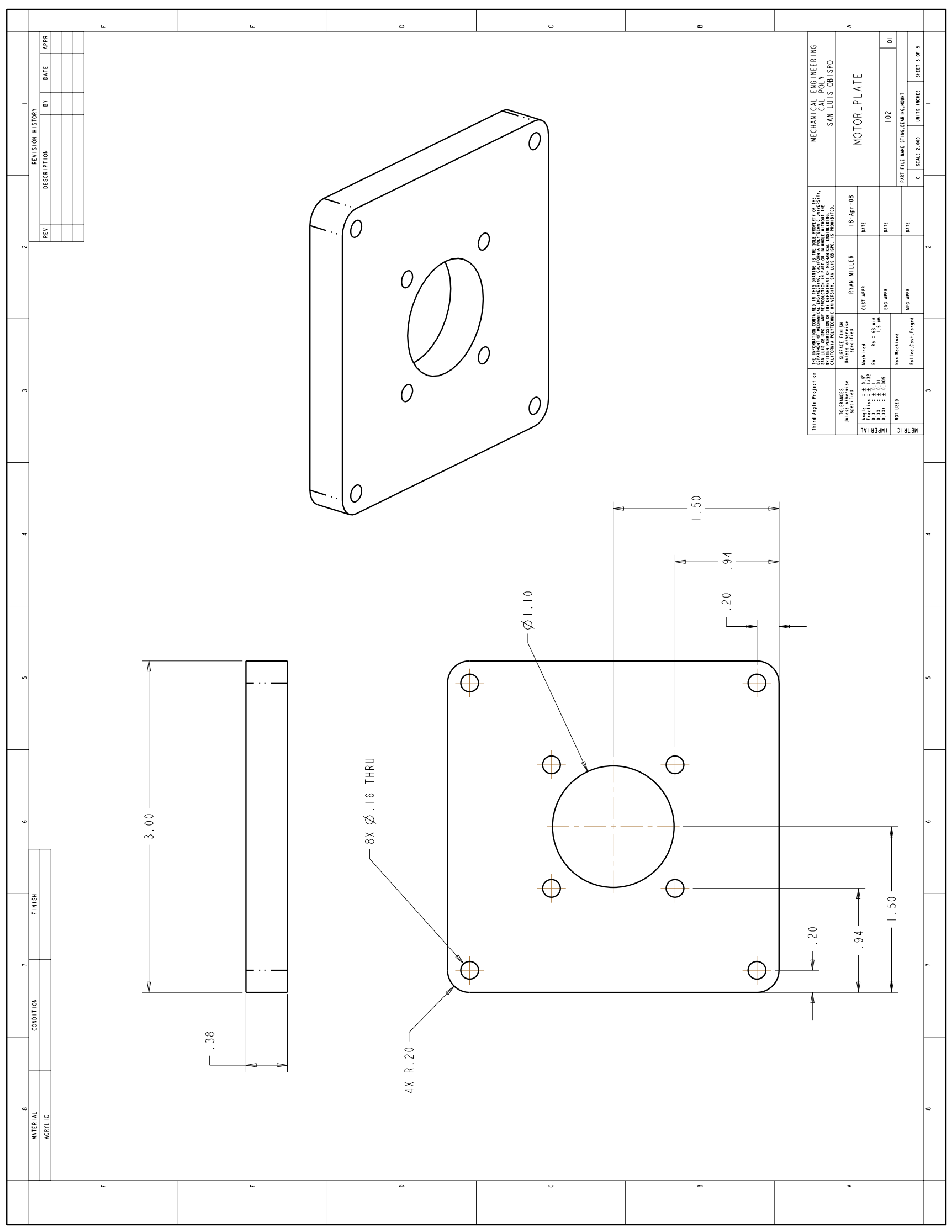




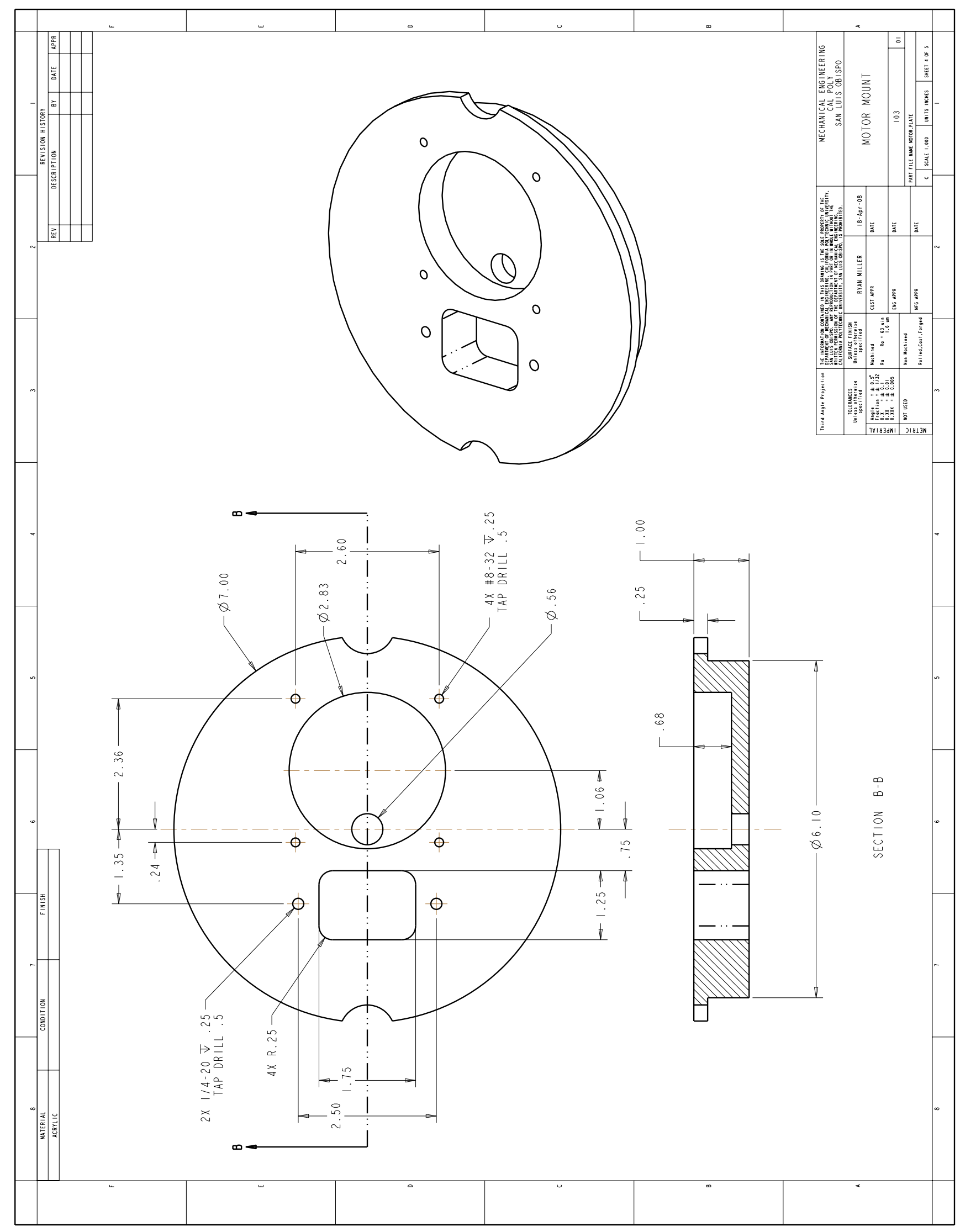




\section{A.2 Initial Concept}

This is the first concept that was developed. These parts were never made;

however, it might be worth exploring in the future. For more information on this design, see Section 2.3. 

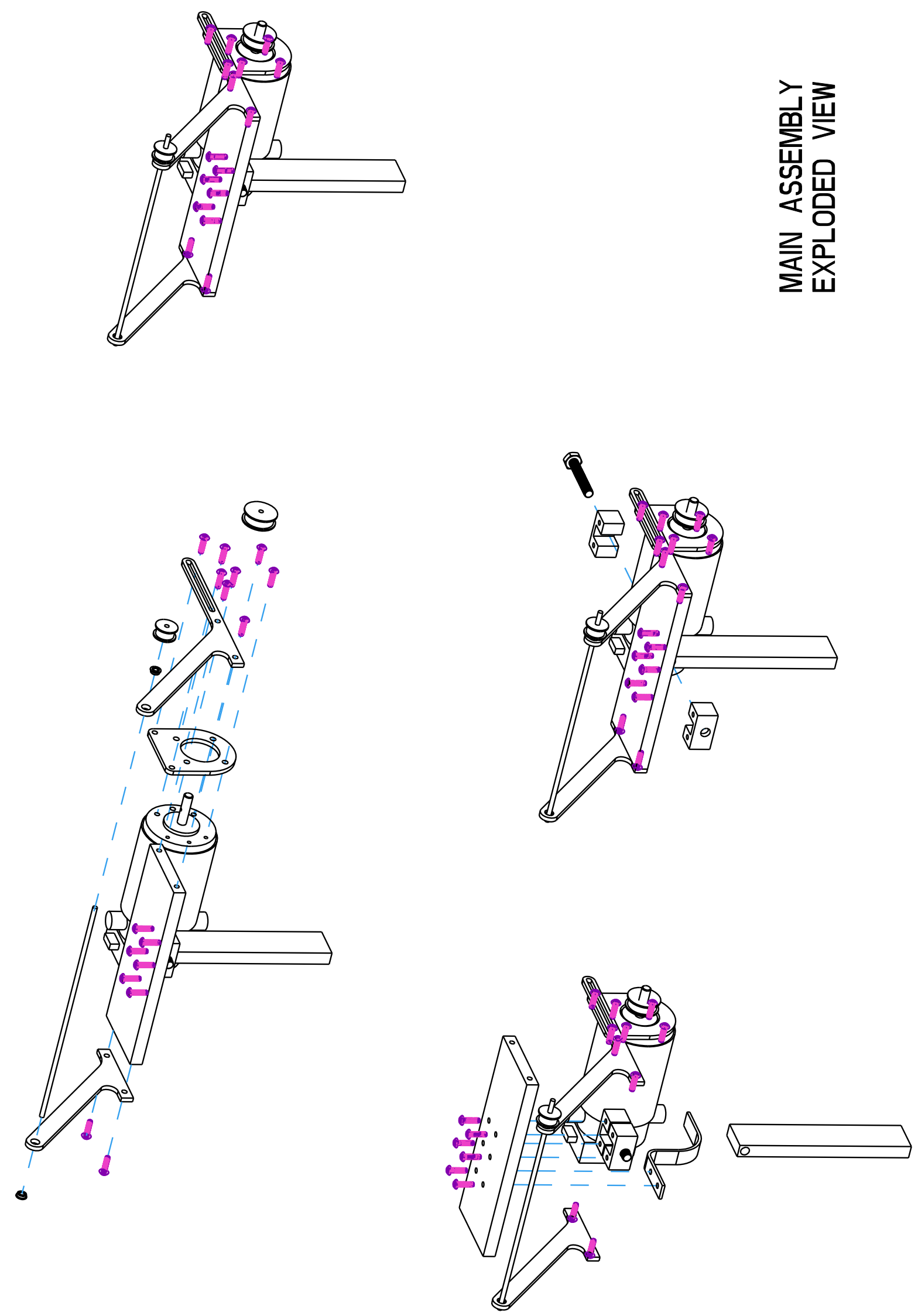


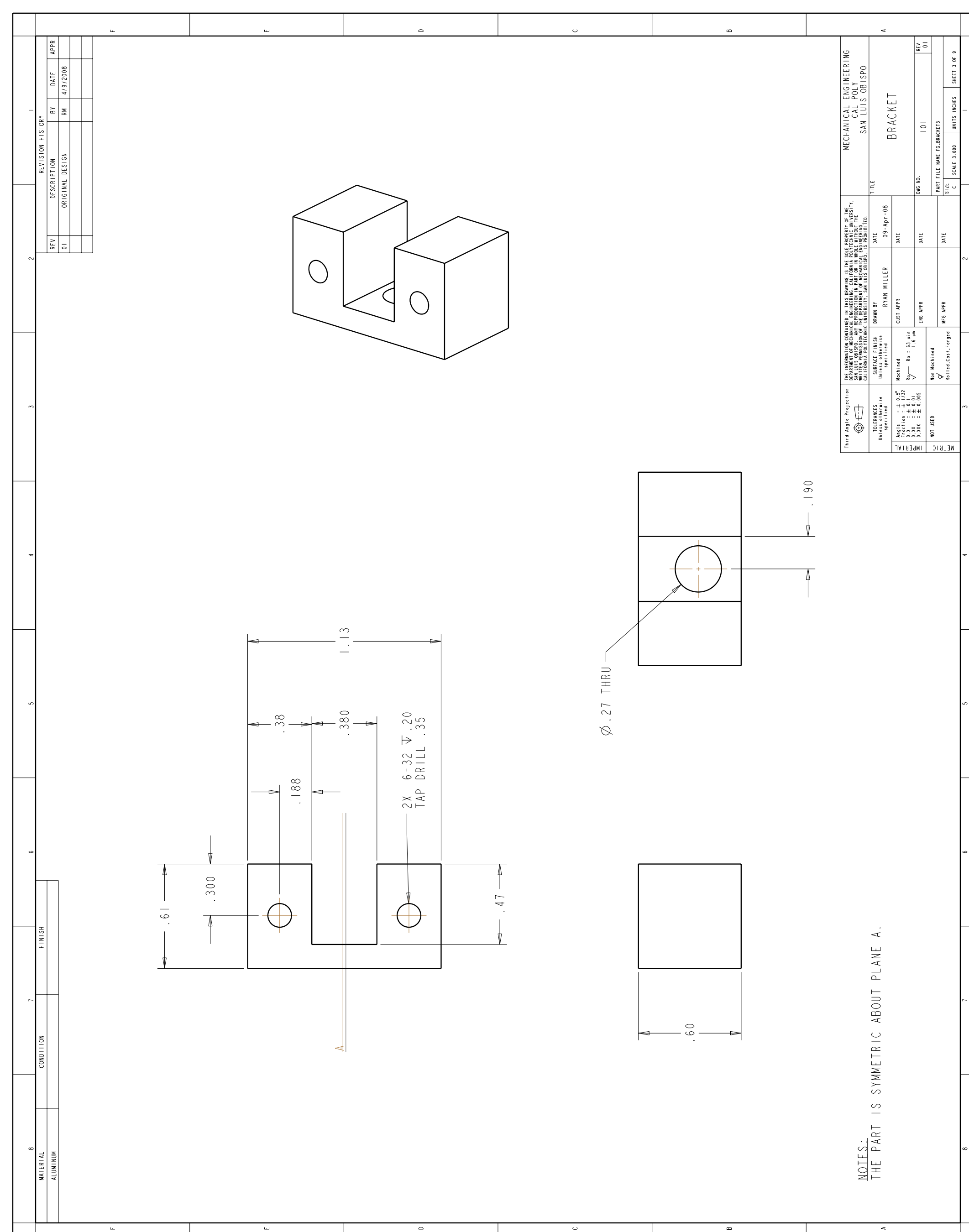





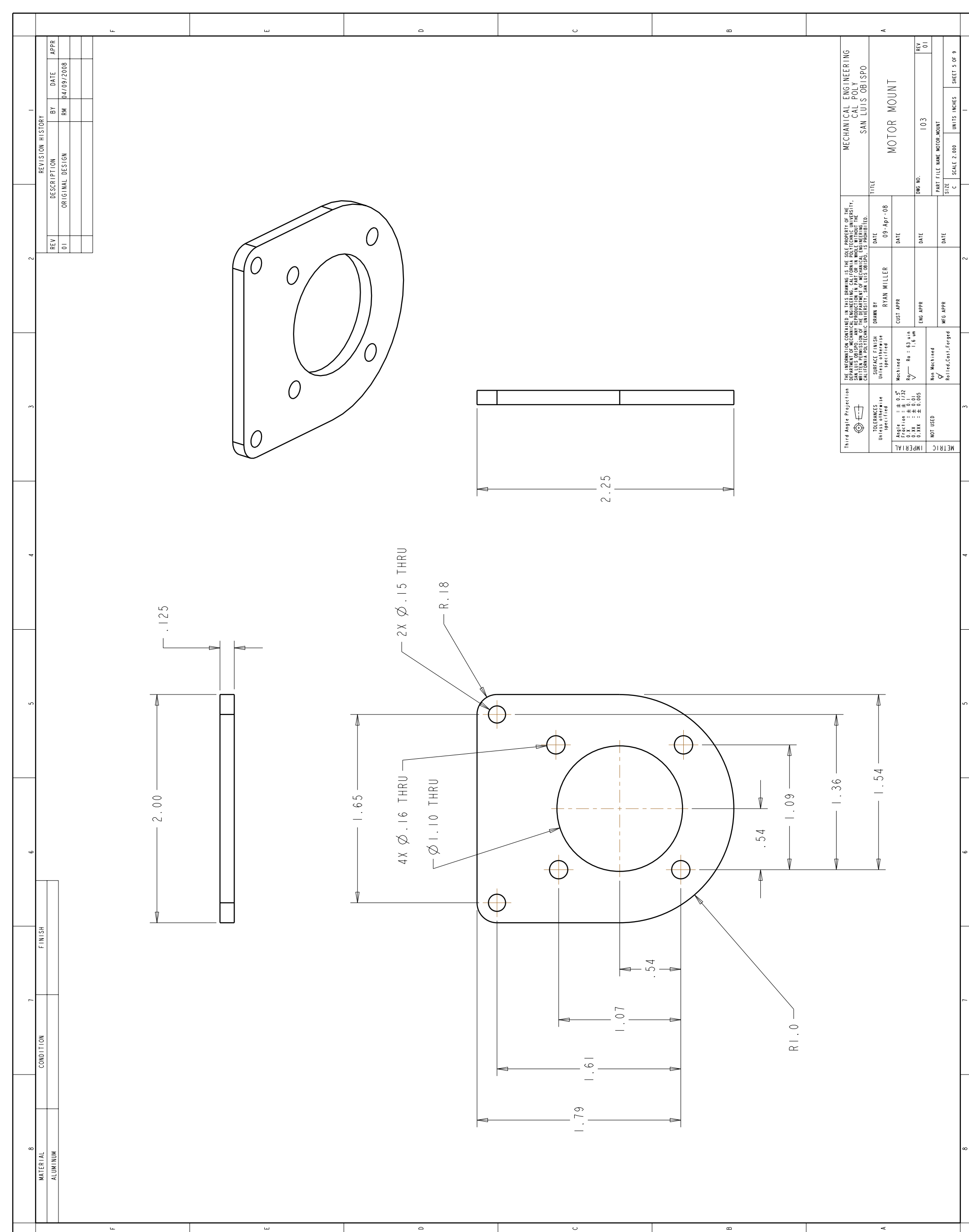




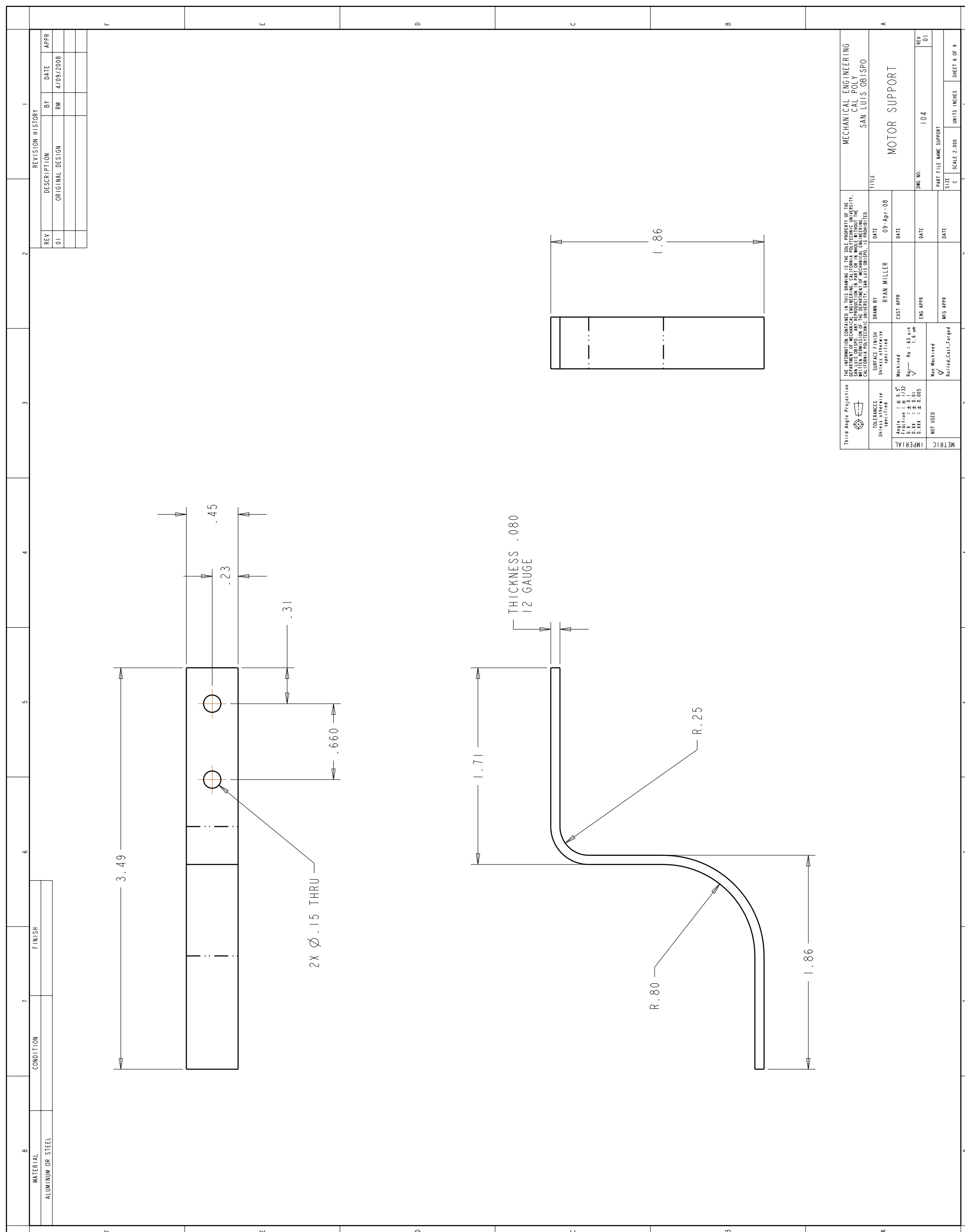




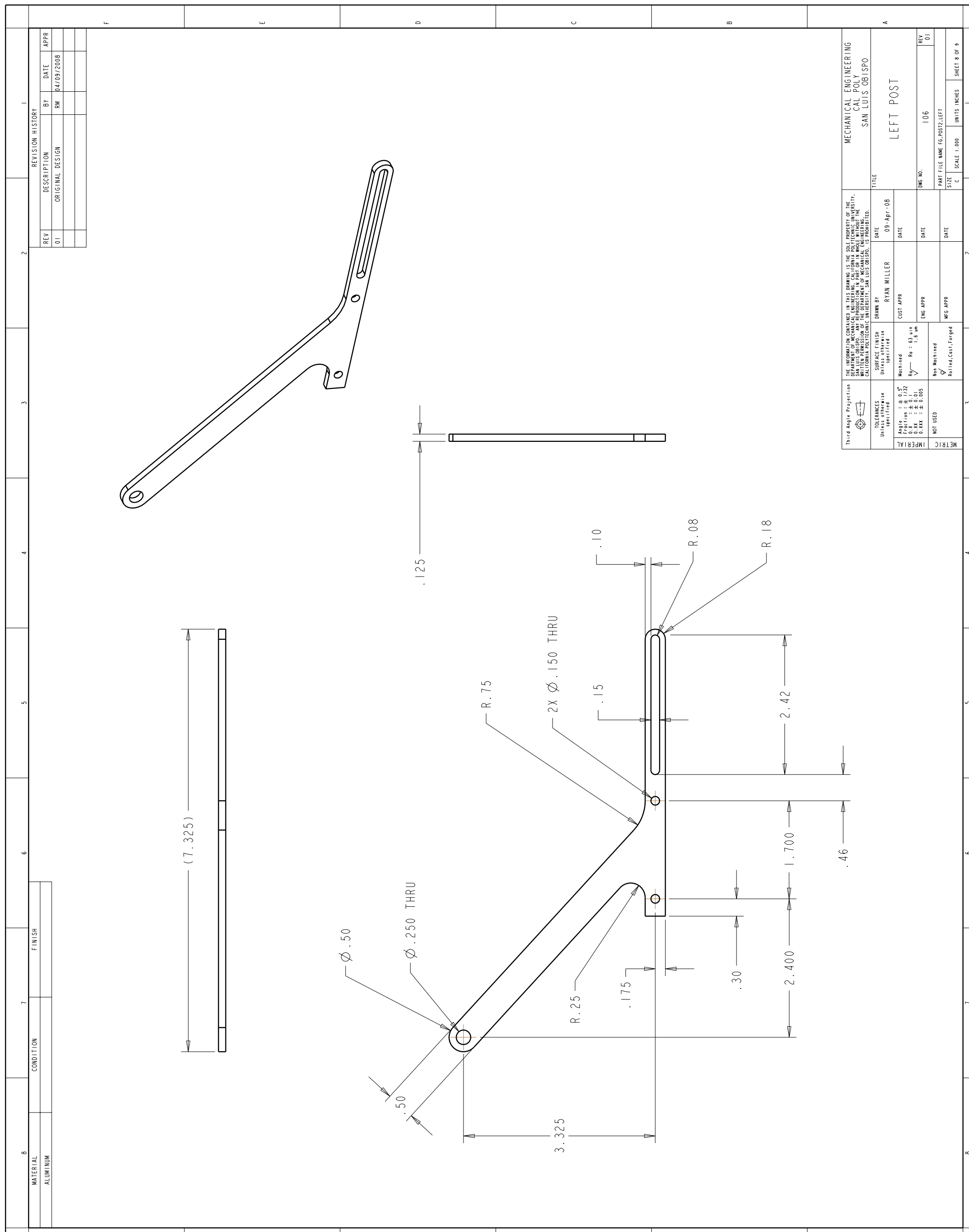




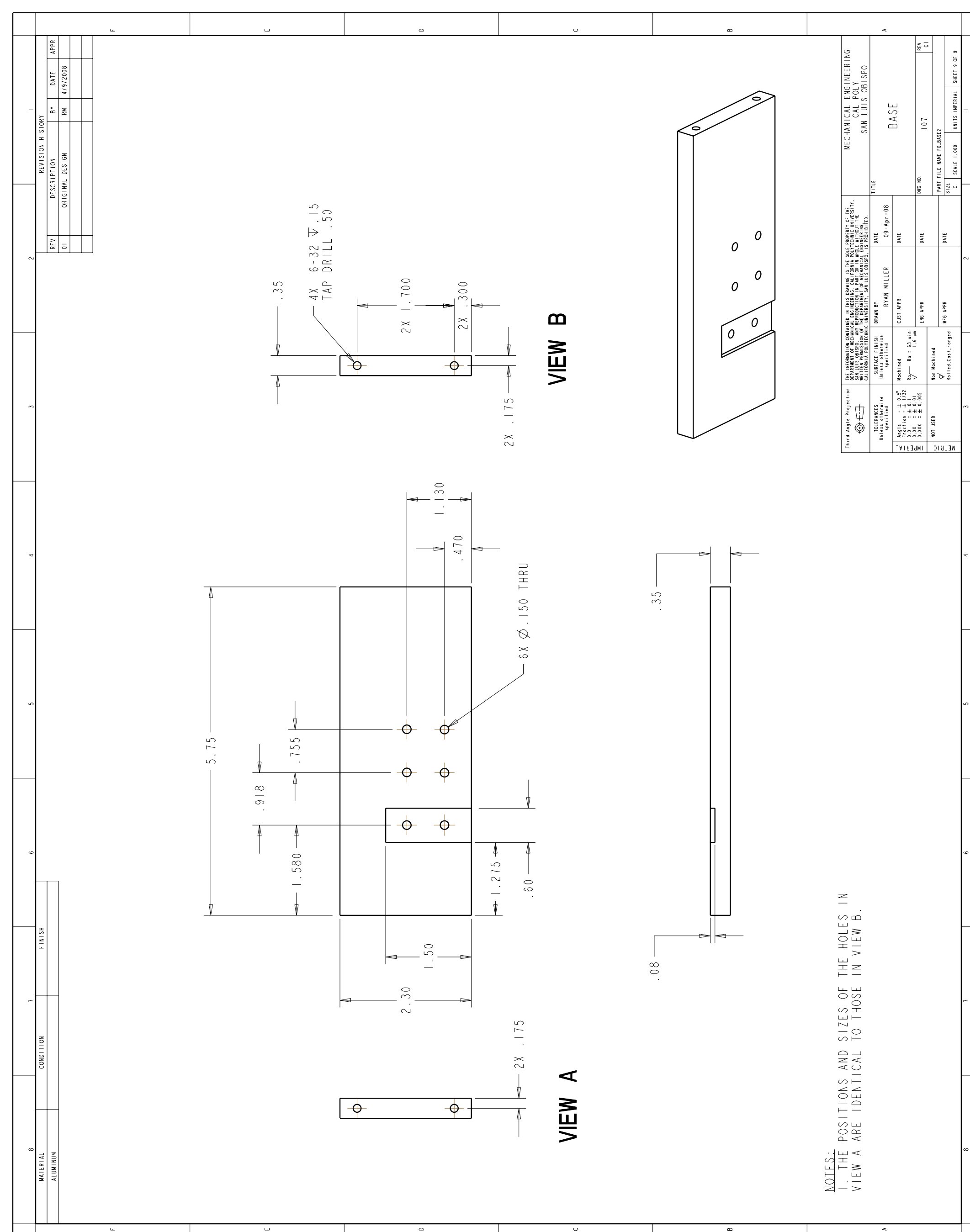




\section{Appendix B}

\section{Mathematics}

\section{B.1 Bernoulli Equation}

$$
\begin{array}{r}
\frac{P}{\gamma}+\frac{V^{2}}{2 g}+z=\frac{P_{0}}{\gamma}+\frac{V_{0}^{2}}{2 g}+z_{0} \\
P-P_{0}=\gamma\left(z_{0}-z\right)+\frac{\rho}{2}\left(V_{0}^{2}-V^{2}\right)
\end{array}
$$

for $z=z_{0}$,

$$
P-P_{0}=\frac{\rho}{2}\left(V_{0}^{2}-V^{2}\right)
$$

From continuity, we know the following.

$$
\begin{array}{r}
Q_{0}=Q_{1} \\
V_{0} A_{0}=V_{1} A_{1} \\
V_{0}=V_{1} \frac{A_{1}}{A_{0}}
\end{array}
$$


Substituting B.2 into B.1 gives,

$$
\begin{aligned}
P_{1}-P_{0} & =\frac{\rho}{2}\left[V_{1}^{2}\left(\frac{A_{1}}{A_{0}}\right)^{2}-V_{1}^{2}\right] \\
& =\frac{\rho}{2} V_{1}^{2}\left[\left(\frac{A_{1}}{A_{0}}\right)^{2}-1\right]
\end{aligned}
$$

This result is very useful since it gives the relationship between pressure and velocity. It has been used throughout the paper to calculate the velocity based on pressure measurements.

\section{B.2 Uncertainty Analysis}

An uncertainty analysis was done to assess whether or not the tests could yield usable information. The analysis shows that the maximum uncertainties associated with the testing procedures are within acceptable ranges. The method is

conducted following Coleman (1999) [7]. To find the uncertainty in the Reynolds number, the following formula is used.

$$
\begin{aligned}
U_{R e}= & \pm\left[\left(U_{P} \frac{\partial R e}{\partial P}\right)^{2}+\left(U_{T} \frac{\partial R e}{\partial T}\right)^{2}+\left(U_{D} \frac{\partial R e}{\partial D}\right)^{2}\right. \\
& \left.+\left(U_{A_{r}} \frac{\partial R e}{\partial A_{r}}\right)^{2}+\left(U_{\Delta P} \frac{\partial R e}{\partial \Delta P}\right)^{2}\right]
\end{aligned}
$$

where $P$ is the ambient pressure of the air, $T$ is the ambient temperature, $D$ is the ball diameter, $A_{r}$ is the contraction area ratio, and $\Delta P$ is the pressure as measured by the Pitot tube. The following assumptions were used to simplify the foregoing result:

1. $R$, the universal gas constant is known to complete accuracy. 
2. The viscocity of air, $\mu$, is solely a function of temperature, that is, $\mu=f(T)$.

3. $\Delta P$ and $P$ are measured separately and are independent variables.

In order to implement this equation, we need to know how to write $R e$ in terms of the previously mentioned variables.

We start with the formulas for the Reynolds number and substitute using the result from Section B.1 and the assumptions from the list.

$$
\begin{aligned}
R e & =\frac{V D}{\nu} \\
& =\sqrt{\frac{2 \Delta P}{\frac{P}{R T}\left(A_{r}^{2}-1\right)}}(D) \frac{1}{f(T)}
\end{aligned}
$$

Simplifying,

$$
\frac{R e}{\sqrt{2 R}}=\Delta P^{1 / 2} T^{1 / 2} P^{-1 / 2} A_{r}^{-1} f(T)^{-1} D
$$

Now to solve for $U_{R e}$ by substituting equation B.7 into equation B.4 and taking partials.

$$
\begin{aligned}
\frac{U_{R e}^{2}}{2 R} & =\left[U_{P} \Delta P^{1 / 2} T^{1 / 2}\left(-\frac{1}{2} P^{-3 / 2}\right) A_{r}^{-1} f(T)^{-1} D\right]^{2} \\
& +\left[U_{T} \Delta P^{1 / 2}\left(\frac{1}{2} T^{-1 / 2}\right) P^{-1 / 2} A_{r}^{-1} f(T)^{-1} D\right]^{2} \\
& +\left[U_{D} \Delta P^{1 / 2} T^{1 / 2} P^{-1 / 2} A_{r}^{-1} f(T)^{-1}\right]^{2} \\
& +\left[U_{A_{r}} \Delta P^{1 / 2} T^{1 / 2} P^{-1 / 2}\left(-A_{r}^{-2}\right) f(T)^{-1} D\right]^{2} \\
& +\left[U_{\Delta P}\left(\frac{1}{2} \Delta P^{-1 / 2}\right) T^{1 / 2} P^{-1 / 2} A_{r}^{-1} f(T)^{-1} D\right]^{2} \\
& +\left[U_{f(T)} \Delta P^{1 / 2} T^{1 / 2} P^{-1 / 2} A_{r}^{-1}\left(-f(T)^{-2}\right) D\right]^{2}
\end{aligned}
$$


Now, we divide by $R e^{2}$ to non-dimensionalize the equation.

$$
\begin{aligned}
\frac{U_{R e}}{R e^{2}} & =\left[U_{P}\left(-\frac{1}{2} P^{-3 / 2}\right) P^{1 / 2}\right]^{2} \\
& +\left[U_{T}\left(\frac{1}{2} T^{-1 / 2}\right) T^{-1 / 2}\right]^{2} \\
& +\left[U_{D} D^{-1}\right]^{2} \\
& +\left[U_{A_{r}}\left(-A_{r}^{-2}\right) A_{r}\right]^{2} \\
& +\left[U_{\Delta P}\left(\frac{1}{2} \Delta P^{-1 / 2}\right) \Delta P^{-1 / 2}\right]^{2} \\
& +\left[U_{f(T)}\left(-f(T)^{-2}\right) f(T)\right]^{2}
\end{aligned}
$$

Simplifying, we obtain the desired expression.

$$
\begin{aligned}
\frac{U_{R e}}{R e^{2}} & =\frac{1}{4}\left(\frac{U_{P}}{P}\right)^{2}+\frac{1}{4}\left(\frac{U_{T}}{T}\right)^{2}+\left(\frac{U_{D}}{D}\right)^{2} \\
& +\left(\frac{U_{A_{r}}}{A_{r}}\right)^{2}+\frac{1}{4}\left(\frac{U_{\Delta P}}{\Delta P}\right)^{2}+\left(\frac{U_{f(T)}}{f(T)}\right)^{2}
\end{aligned}
$$

To calculate a numerical value for the Reynolds number uncertainty, the following the values are used.

1. $U_{A_{r}}=0.01$. This number is from the tolerance listed in the wind tunnel operation manual published by the OEM [15].

2. $U_{\Delta P}=0.001$. The original display for the pressure reading indicates that the value is good to three decimal places.

3. $U_{T}=1$ degree $F$. The thermostat in the room reads the temperature in increments of 1 degree F.

4. $U_{f(T)} / f(T)=0.01$. This is estimated.

5. $U_{P}=0$, since this value is established from atmospheric pressure data. 
6. $U_{D} / D=0.02$. Estimated from size and weight requirements put forth by the USGA [33]. The USGA only specifies a minimum diameter, not a maximum. The ball is restricted to a maximum weight, however, of 1.620 ounces avoirdupois.

By inserting these values into the above expression, the total uncertainty is found to be,

$$
\frac{U_{R e}}{R e}=2.4 \%
$$

This amount of uncertainty is acceptable given that fluid mechanics measurements involving transition phenomena are generally only accurate to $5-10 \%$ [13]. 


\section{Appendix C}

\section{Software Tools}

Screenshots of the various different software tools are presented below for the main purpose of letting any user recognize the program. These are the programs that were used for the purpose of data acquisition. These programs should be preserved in a folder called Wind Tunnel DAQ on the desktop of the computer used for data acquisition linked to the wind tunnel used for this study. The file names of the programs are included in the caption of the figure when applicable. 


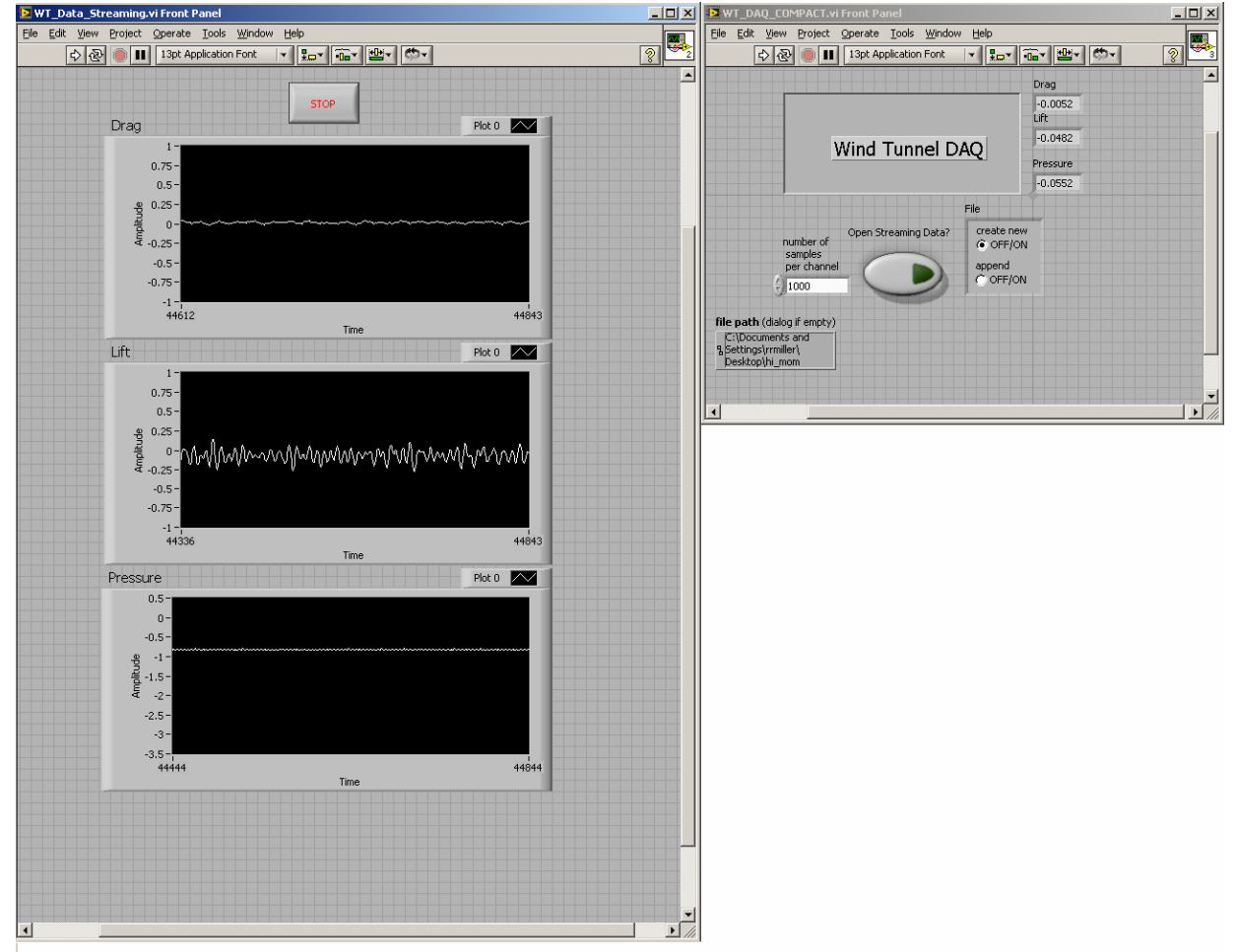

Figure C.1: LabVIEW GUI as it would be when the wind tunnel is running. The three graphs on the left are streaming data of drag, lift, and pressure. This program is called WT_Data_Streaming.vi. The window on the right is the interface for the data acquisition functionality, called WT_DAQ_COMPACT.vi. 

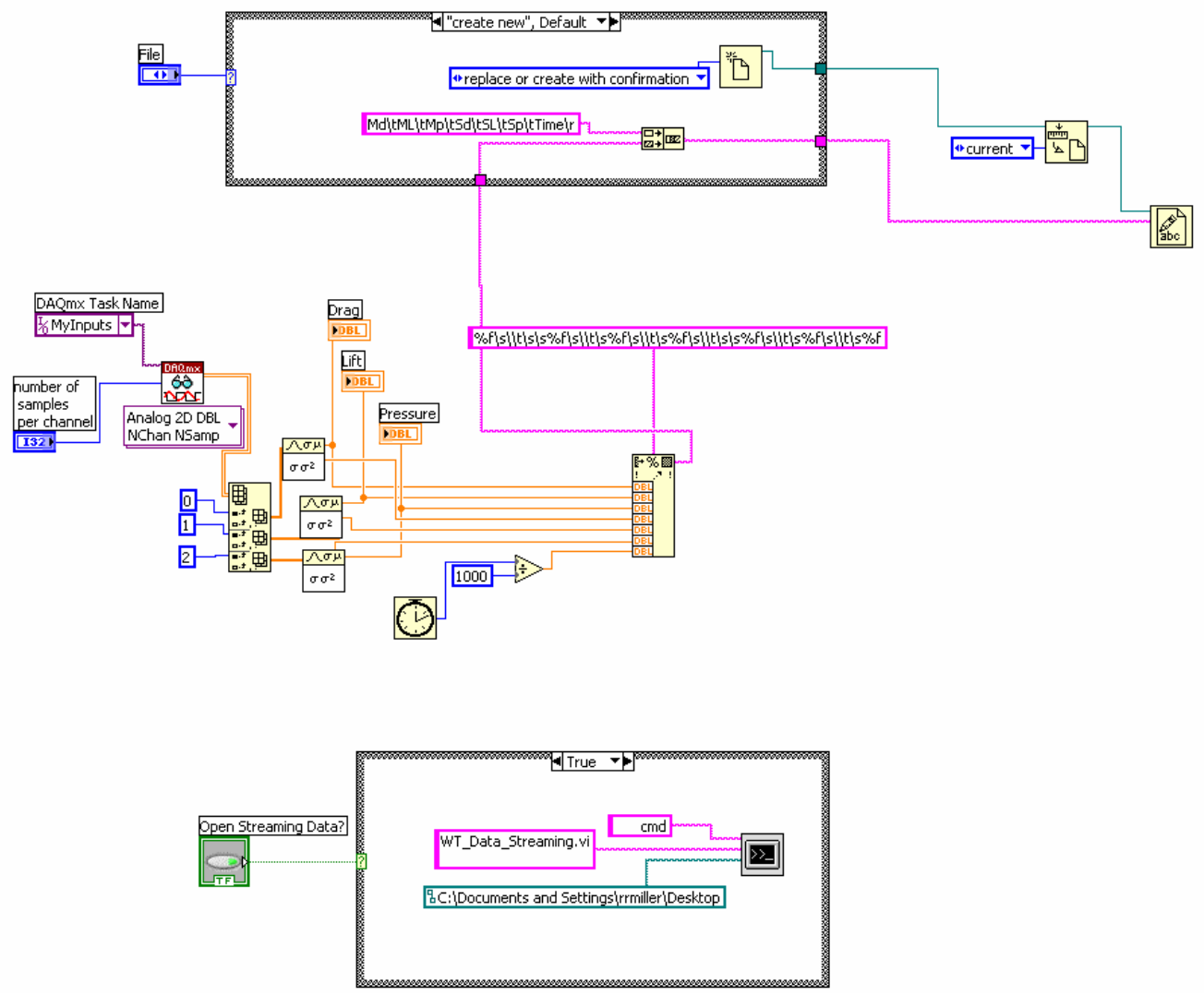

Figure C.2: The block diagram for the LabVIEW program that records data. WT_DAQ_COMPACT.vi. 


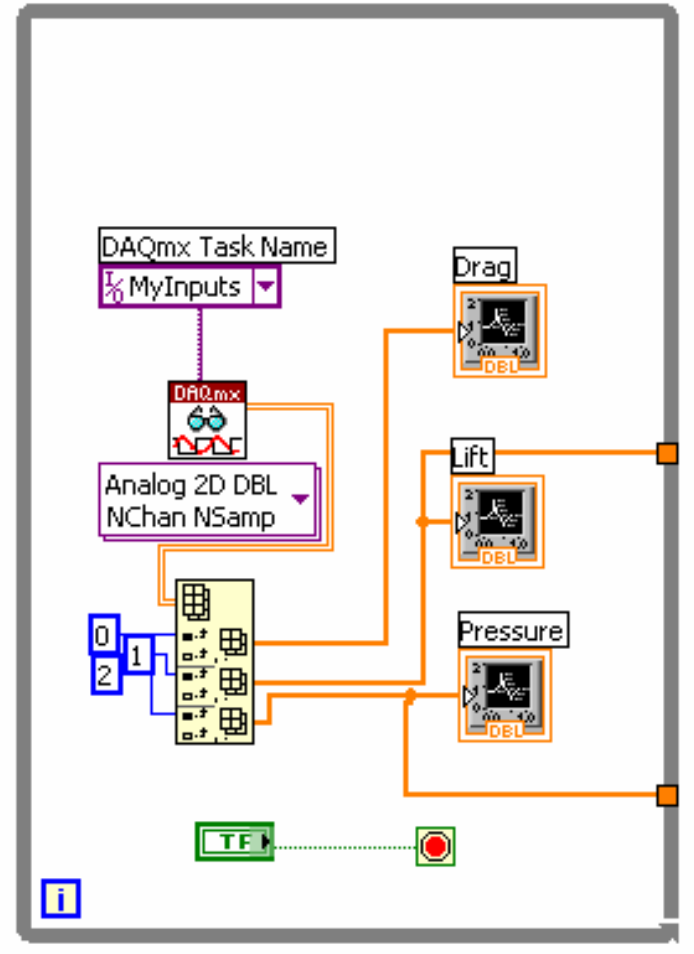

Figure C.3: The block diagram for the LabVIEW program that displays data in real-time. WT_Data_Streaming.vi. 


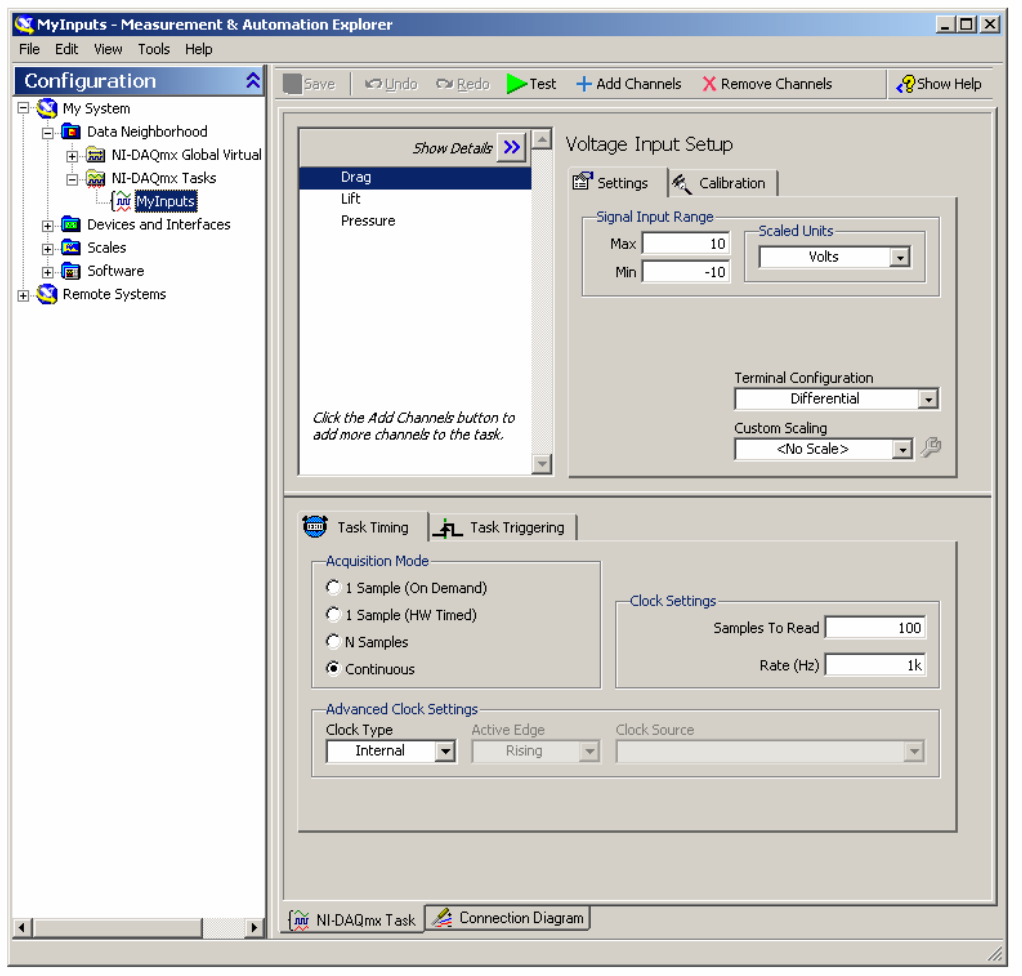

Figure C.4: This is a screenshot of the window that controls the hardware that LabVIEW utilizes, called Measurement $\&$ Automation. 


\section{Appendix D}

\section{Calibration}

Prior to testing, the sting and the pressure transducer were calibrated. The following calibration curves were generated. These curves are highly repeatable, indicating that, at their core, these systems are quite capable of recording data of high integrity. 


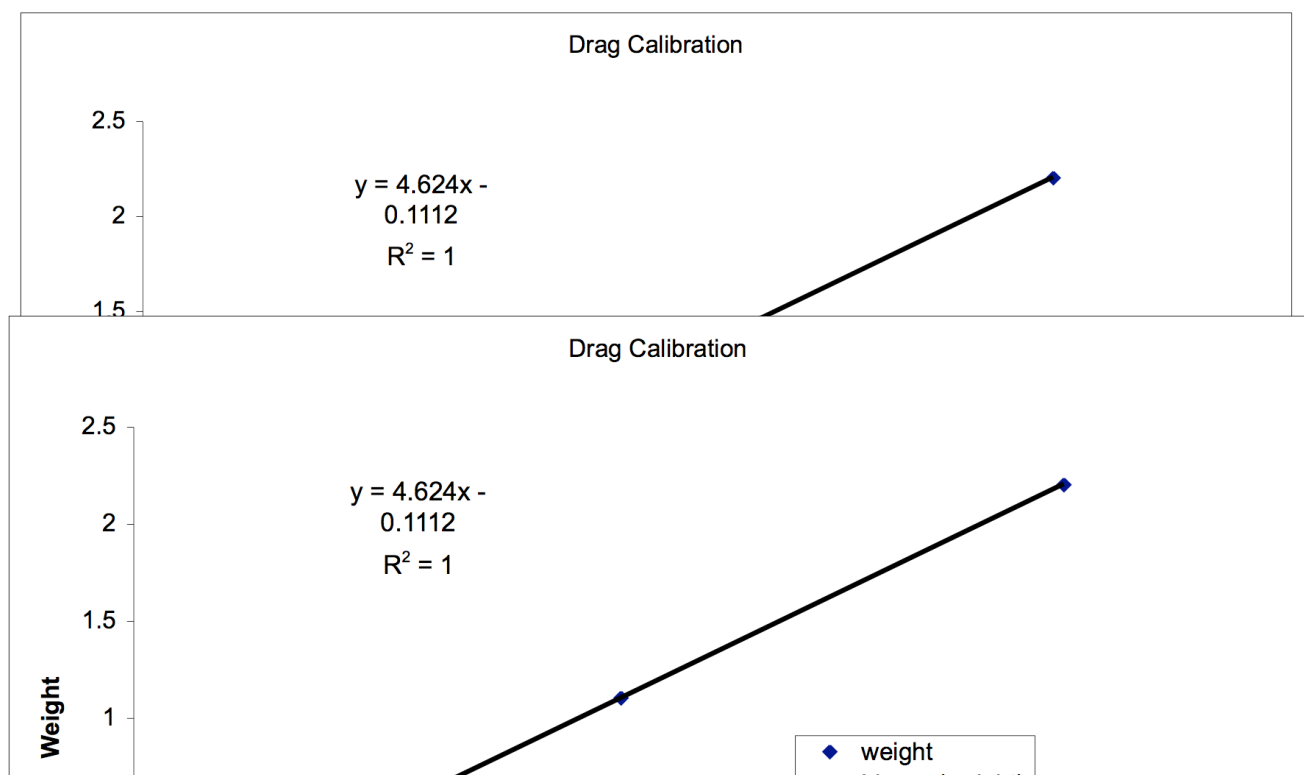

Figure D.1: Drag calibration curve.

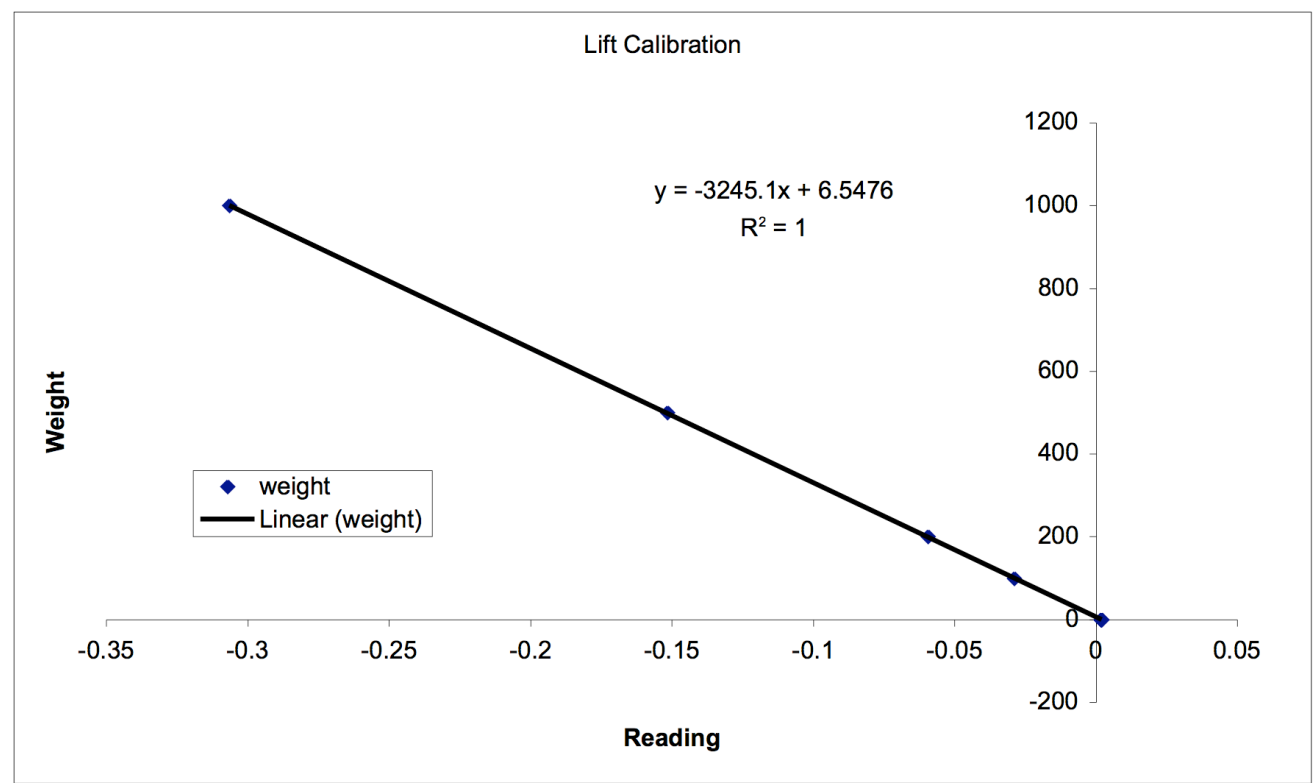

Figure D.2: Lift calibration curve. 


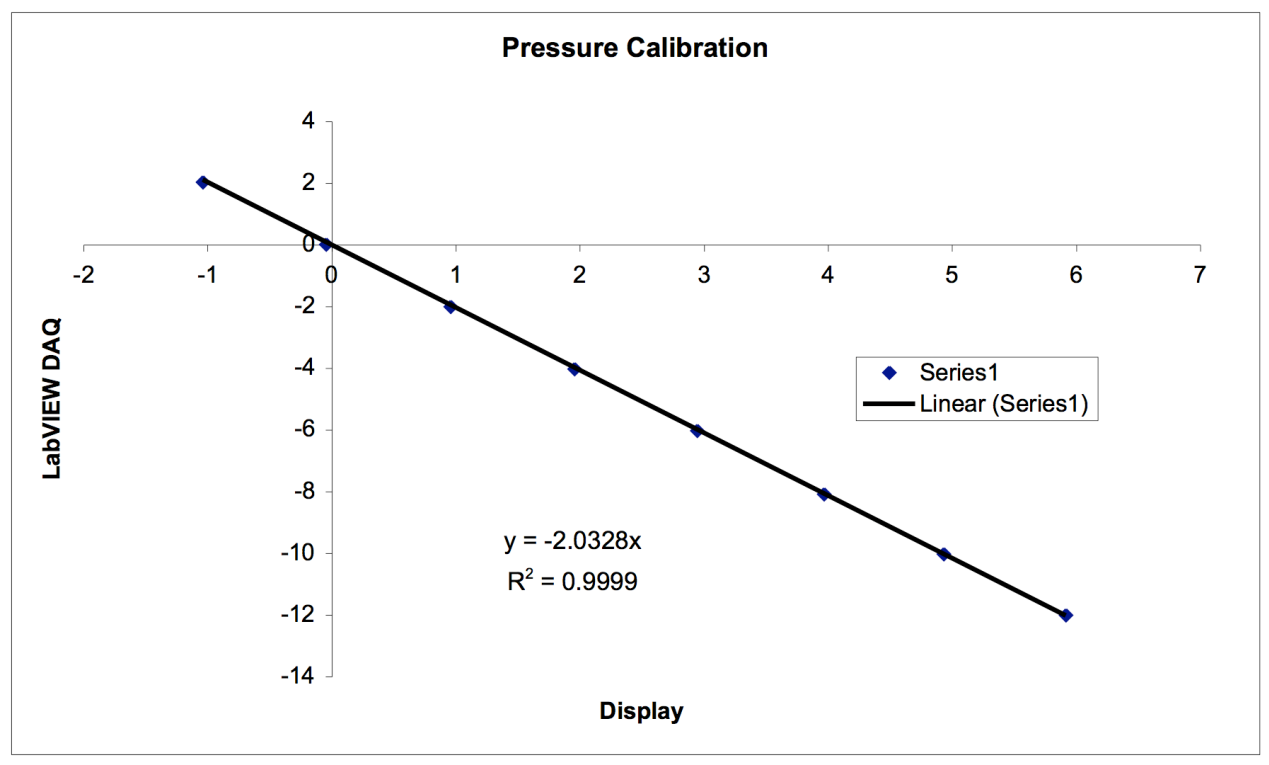

Figure D.3: Pressure calibration curve. 